\title{
An illustrated key to male Actinote from Southeastern Brazil (Lepidoptera, Nymphalidae)
}

\author{
Ronaldo Bastos Francini ${ }^{1}$ \& Carla M. Penz ${ }^{2}$
}

Biota Neotropica v6 (n1) - http://www.biotaneotropica.org.br/v6n1/pt/abstract?identification-key+bn00606012006

\author{
Date Received 06/06/2005 \\ Revised10/20/2005 \\ Accepted 01/01/2006
}

${ }^{1}$ Universidade Católica de Santos, Av. Cons. Nébias 300, 11015-200 Santos, SP, Brazil; email: francini@unisantos.br

${ }^{2}$ Department of Biological Sciences, University of New Orleans, 2000 Lakeshore Drive, New Orleans, LA 70148, USA, email: cpenz@uno.edu. Associate professor, Programa de Pós-Graduação em Biociências, Pontifícia Universidade Católica do Rio Grande do Sul, Av. Ipiranga 6681, 90619-900, Porto Alegre, RS, Brazil.

\begin{abstract}
Francini, R.B. and Penz, C.M. An illustrated key to male Actinote from Southeastern Brazil (Lepidoptera, Nymphalidae). Biota Neotrop. Jan/Abr 2006, vol. 6, no. 1 http://www.biotaneotropica.org.br/v6n1/pt/abstract?identificationkey+bn00606012006. ISSN 1676-0603

In certain times of the year, Actinote butterflies can be vastly abundant in Southeastern Brazil, thus representing conspicuous denizens of our fauna. Their wing coloration allows for the recognition of five major patterns, which probably resulted from mimicry. Furthermore, examination of series of field-collected and reared specimens clearly demonstrates a high intra-specific variation in wing color pattern. In concert, inter-specific resemblance (mimicry) and intraspecific variation make it difficult to discriminate Actinote species from each other. This key aims at providing a tool for identification of male Actinote. To that effect, we provide diagnostic characters and illustrations of wings and male genitalia for 22 species. We hope that the clear identification of these species will lead to the discovery of species yet unrecognized in Southeastern Brazil.
\end{abstract}

Key words: butterfly, identification, genitalia, mimicry.

\section{Resumo}

Francini, R.B. and Penz, C.M. Chave ilustrada para machos de Actinote do sudeste do Brasil (Lepidoptera, Nymphalidae). Biota Neotrop. Jan/Abr 2006, vol. 6, no. 1 http://www.biotaneotropica.org.br/v6n1/pt/abstract?identificationkey+bn00606012006. ISSN 1676-0603

Em certas épocas do ano, as borboletas do gênero Actinote podem ser muito abundantes no sudeste do Brasil, constituindo, então, elementos conspícuous da nossa fauna. Sua coloração alar permite a separação em cinco padrões básicos, provavelmente relacionados com o mimetismo. Além disso, séries de exemplares coletados no campo ou criados em laboratório demonstram claramente que existe uma grande variabilidade intra-específica na coloração alar destas borboletas. Em conjunto, a semelhança entre as espécies (mimetismo) e variação intra-específica dificultam o reconhecimento das espécies de Actinote. Esta chave tem como objetivo auxiliar a identificação dos machos destas borboletas e, para tanto, são apresentados caracteres diagnósticos, ilustrações das asas e genitália de 22 espécies. Esperamos que a clara identificação destas espécies possa revelar a presença de espécies ainda não reconhecidas no sudeste do Brasil.

Palavras-chave: borboleta, identificação, genitalia, mimetismo. 


\section{Introduction}

The butterfly tribe Acraeini (Nymphalidae, Heliconiinae) currently holds the African genera Pardopsis Trimen, 1887, Acraea Fabricius, 1807 and Bematistes Hemming, 1935, plus the Neotropical Actinote Hübner, [1819], Abananote Potts, 1943 and Altinote Potts, 1943 (Ackery 1984, 1988, Penz \& Djunijanti 2003, Lamas 2004). Ehrlich (1958), Van Son (1963), and Penz \& Djunijanti (2003) provided several characters to define this group, including the closed hindwing cells, stalking of the forewing radial veins, morphology of dorsal forewing scales, forelegs, genitalia, and first instar larvae. Independently of the continent one happens to visit, or color pattern details, acraeines can be easily recognized in the field by their elongated wings and flight behavior - the butterfly equivalent of recognizing 'robins' or 'figs' as natural groups of organisms.

As a taxonomic unit, the generic status of Actinote is uncertain. In a comprehensive phylogenetic study of Acraeini, Pierre (1987) suggested that Actinote nests within and should be regarded a subgenenus of Acraea. Although Pierre's hypothesis has been recently supported with a small sample of species (Penz \& Djunijanti 2003), from a practical standpoint it is unclear if Actinote and other Neotropical genera should be subsumed within Acraea (Pierre's approach) or if the speciose Acraea should be divided into smaller monophyletic units (Actinote being one of them, according to Pierre). Therefore, the generic rank of Actinote has been maintained here for tradition and convenience, despite the fact that these Neotropical butterflies are likely closely related to a discrete subset of African Acraea.

Several natural history characteristics distinguish Actinote from other Neotropical heliconiines. Males produce a mating plug, which they attach to the females after copulation (Ehrlich \& Ehrlich 1978, Paluch et al. 2003). Eggs are always laid in clusters, and larval cycles are usually long, with some species being univoltine and spending part of the year in diapause as early instar larvae (Francini 1989, 1992 and unpublished. obs.). Adults can reach high abundances in SE Brazil in late Spring (October-November) and early Fall (MarchApril), but some univoltine species can be quite rare in collections because of their short adult life and limited geographic distribution (e.g., A. zikani; Francini 1992). While several heliconiine species prosper inside the Amazon forest, Actinote species often prefer open habitats where their Asteraceae host plants also thrive. In southeastern Brazil, they collectively inhabit an altitudinal range from sea level to $2400 \mathrm{~m}$.
Species of Actinote use toxic chemical compounds and mimicry as protection against predation (e.g., Müller 1879, Brown \& Benson 1974, Francini 1989, 1992). Cyanogenic glycosides and pyrrholizidine alkaloids are present in all life stages (Francini 1989, Brown \& Francini 1990). Their lethargic flight, elongated abdomen and often aposematic coloration are characteristic of chemically protected butterflies (e.g., Bates 1862, Poulton 1902, Chai 1986, 1996, Srygley 1994). The 22 Actinote species in SE Brazil considered here are members of five different mimicry rings (Francini 1989, 1992, Brown \& Francini 1990), and wing coloration for any given species is probably a result from the combined history of their phylogenetic and mimetic associations.

The dynamics of mimicry groups within Actinote strongly contrasts their relatives in the genus Heliconius Kluk, 1780. In southeastern Brazil, Actinote species from different mimicry groups usually fly together in the same area, and broadly overlap in adult resource use (nectaring plants, basking spots) and activity patterns. Furthermore, conspecific individuals may vary considerably in the size and color of their forewing markings both within and between sexes. At a community level, therefore, the end result potentially includes a mixed group of individuals that show a range of forewing patterns from dark-orange to white to semi-translucent in random proportions. This commonly seen Actinote mishmash greatly contrasts Müllerian mimicry rings of Heliconius, where both field observations and theory suggest that Müllerian mimics should, and do, resemble one another as closely as possible (e.g., Turner 1987). Nothing is known about how potential predators perceive and react to color variation in Actinote.

Although as a group Actinote butterflies constitute an instantly recognizable component of the butterfly fauna in Southeastern Brazil, species identification is often problematic (e.g., Hayward 1931). This difficulty is due to the interaction between two factors: some Actinote species share extremely similar wing patterns; and all species have a high level of intraspecific variation. Furthermore, females of several species are nearly indistinguishable from one another (D'Almeida 1935a, Francini 1989). As a result, the often-incomplete series of specimens available in early collections, abbreviated descriptions, and limited illustrations led to much confusion regarding species identification (e.g., see synonymy for A. pyrrha (Fabricius), below). 
Here we present an illustrated key for the identification of male Actinote from southeastern Brazil based on 25 years of fieldwork and comparative morphology. The São Paulo region has the highest species richness for Actinote due to habitat diversity and overlapping species distributions. Therefore, our geographical focal point is São Paulo and surrounding states, where the 'Mata Atlântica' (Atlantic forest) vegetation ranges from open 'restinga' (sea level, shrubby vegetation neighboring the ocean), to cloud forest (2000 m). We provide synonymy, a brief diagnosis for males of each species included in the key, geographical distribution of the species, and illustrations of male genitalia.

\section{Material and Methods}

We attempted to study Actinote populations both in the field and in the laboratory. Because females lay eggs in clusters, sib groups are useful for evaluating intraspecific variation and for matching males and females of the same species - which gave us particularly valuable insight to variation and overlap between A. pellenea Hübner and carycina Jordan. There is a cost, however, to studying organisms that flourish in second growth or naturally open habitats. Although Actinote carycina larvae are common in second growth and roadsides where their Asteraceae host plants grow in abundance, their populations (and our samples) were often decimated by road clearings. Furthermore, some species are difficult to maintain in culture. For example, Actinote morio Oberthür is univoltine, has a long life cycle and feeds on a single Asteraceae host plant that can only be found $300 \mathrm{~km}$ from the laboratory. Despite these difficulties, we examined male external and internal morphology of both field-collected and laboratory reared adults to provide characters for species identifications.

Color variation in Actinote gave rise to the description of numerous subspecies and varieties. Although some of the described subspecies may represent legitimate divergence (see Species Accounts section), many of the names that have been proposed to define geographic races actually correspond to variation that can be found within a single population and are, thus, biologically meaningless. Here, therefore, our focus is to define and identify Actinote butterflies to the species level, and we defer the study of subspecific divergence to furture workers.
Our long-term experience with series of specimens demonstrates that positive identification of certain species can only be achieved through genitalic examination, which can sometimes be done without dissection even in the field using a good magnifying lens. Dissection of male genitalia followed standard methods of soaking the abdomen in 10\% $\mathrm{KOH}$, and permanent storage in a vial filled with glycerol. For the key, illustrations of the male right valva simply consist of an outline in dorsal, ventral, or lateral views, flattened to a uni-dimensional plane, and devoid of setae, and with the valval base positioned to the left. This was done purposefully for ease of visualization of characters, and detailed drawings of the complete male genitalia are given in the plates that accompany the species accounts. Most, but not all species, are used as examples in the illustrations of characters in the key.

For the species accounts, taxa are listed in the order that they appear in the key, which does not imply phylogenetic relationships. Because the authorities that designated each taxon name are listed in the species accounts, we do not include their names in the key or figure legends. We give the synonymy, diagnosis, geographical distribution, and brief remarks when applicable. The synonymy is as complete as possible, barring records that were dubious, unclear, or unavailable to us. The diagnosis is based on specimens from SE Brazil, and therefore may not reflect the complete color variation found across species ranges. Distributions were based on collection specimens. Each species is illustrated in a plate by pictures of the wings (dorsal) and scales (ventral hindwing, basal area), and drawings of male genitalia (dorsal, ventral and lateral views; anterior portion to the left). Further information about nomenclature, type specimen deposition, synonymy and distribution can be found in Lamas (1996, 2004).

We examined specimens in the following collections: RBF (Ronaldo Francini's personal collection, currently at the Museu de Zoologia, Universidade de São Paulo, São Paulo, Brazil), UFPR (Universidade Federal do Paraná, Curitiba, Brazil), MAPA (Museu Anchieta, Porto Alegre, Brazil), MPM (Milwaukee Public Museum, Milwaukee, USA). Most fieldwork was done in São Paulo and Rio Grande do Sul states, Brazil, but RBF also visited Paraná, Minas Gerais, Rio de Janeiro, Espírito Santo and Bahia states. 


\section{Illustrated key to male Actinote from southeastern Brazil based on wing pattern and genitalia}

1a. Wings completely opaque and predominantly iridescent-black except for forewing yellow markings [iridescentblack mimicry group, example in Figure 1A]

1b. Certain wing areas translucent to some extent [black-yellow mimicry group, example in Figure1B; greasy-orange mimicry group, example in Figure 1C]

1c. Wings completely opaque but never iridescent-black [orange-yellow mimicry group, example in Figure 1D; red mimicry group, example in Figure 1E]

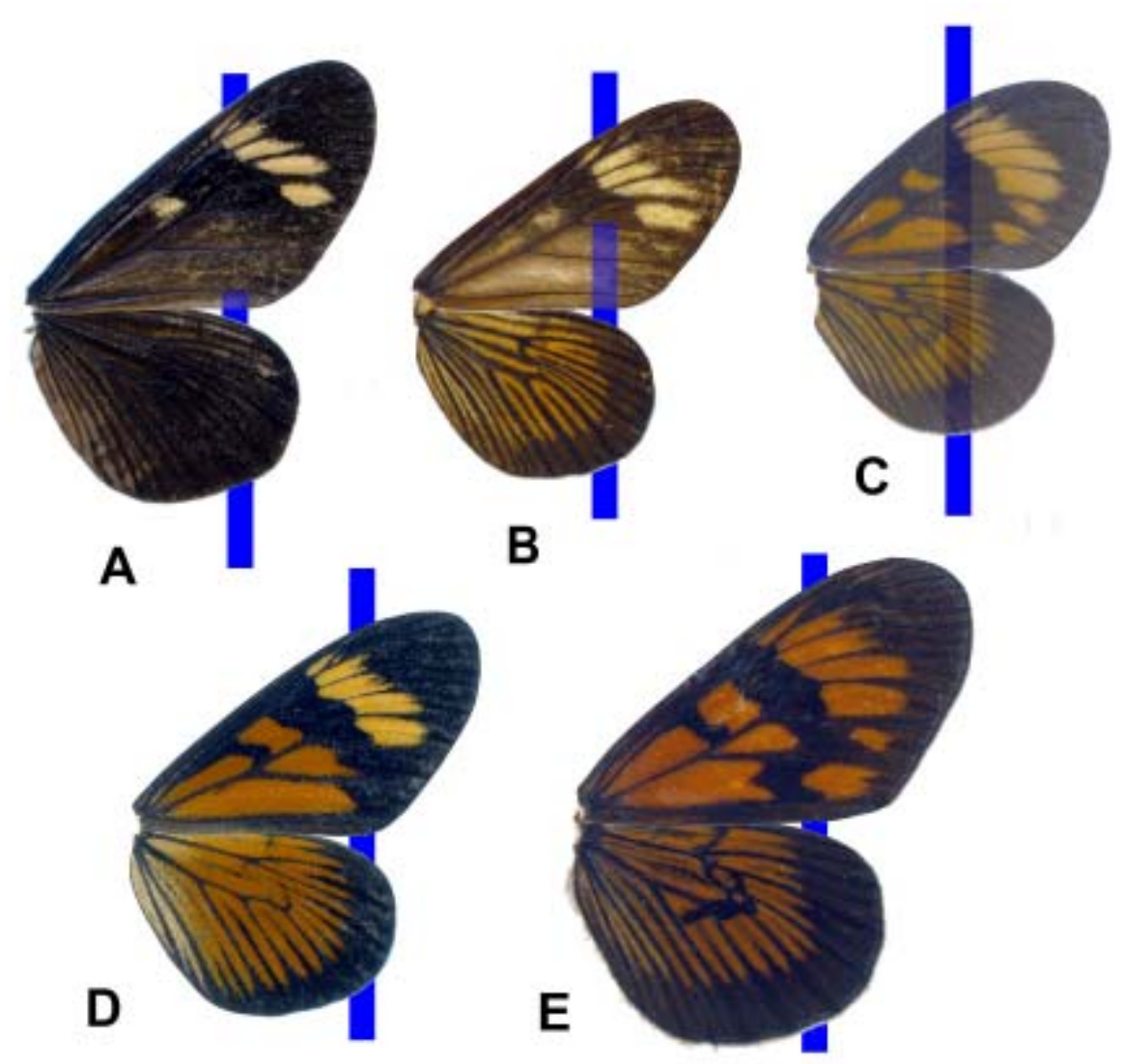

Figure 1. Vertical blue lines help to show the degree of wing transparency. (A) A. zikani; (B) A. melanisans; (C) A. canutia; (D) A. brylla; (E) A. dalmeidai.

2a. Fore- and hindwings largely translucent and with a greasy appearance 

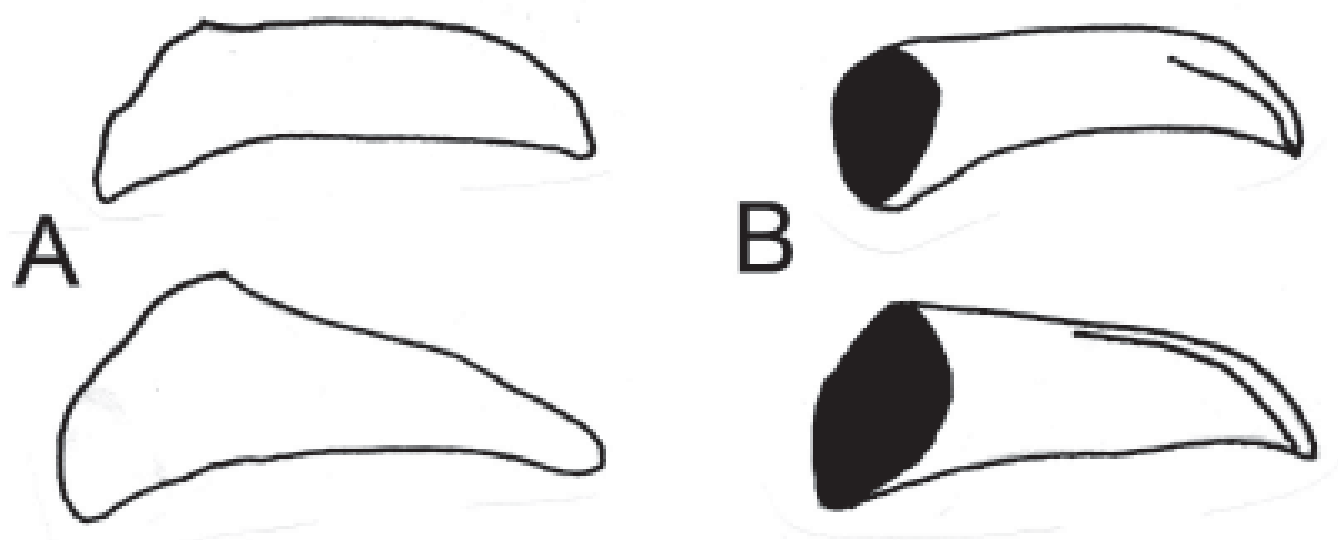

Figure 2. Dorsal and ventral views of the valva, setae ommited. (A) Actinote morio; (B) A. zikani.

4a. Hindwing comma-shaped mark well developed (indicated by the arrow in Figure 3A) . mamita

4b. Hindwing comma-shaped mark faded (Figure 3B) canutia
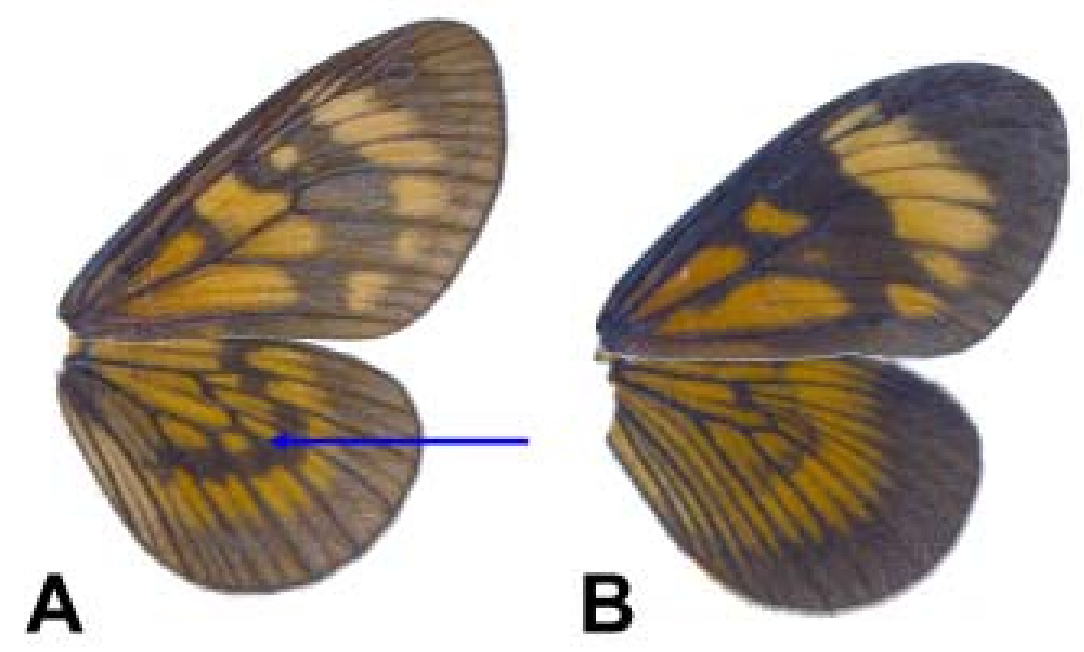

Figure 3. Fore- and hindwngs; dorsal surface. (A) A. mamita, the arrow indicates the comma-shaped mark; (B) A. canutia. 
5a. Valva much longer than broad ( $5 \mathrm{x}$ longer than the maximum width), with tube-like appearance and blunt apex (Figure 4A); forewing greasy and strongly translucent at base (Figure 4B) genitrix

5b. Valva less than $3 x$ as long as it is wide (maximum length/maximum width) 6
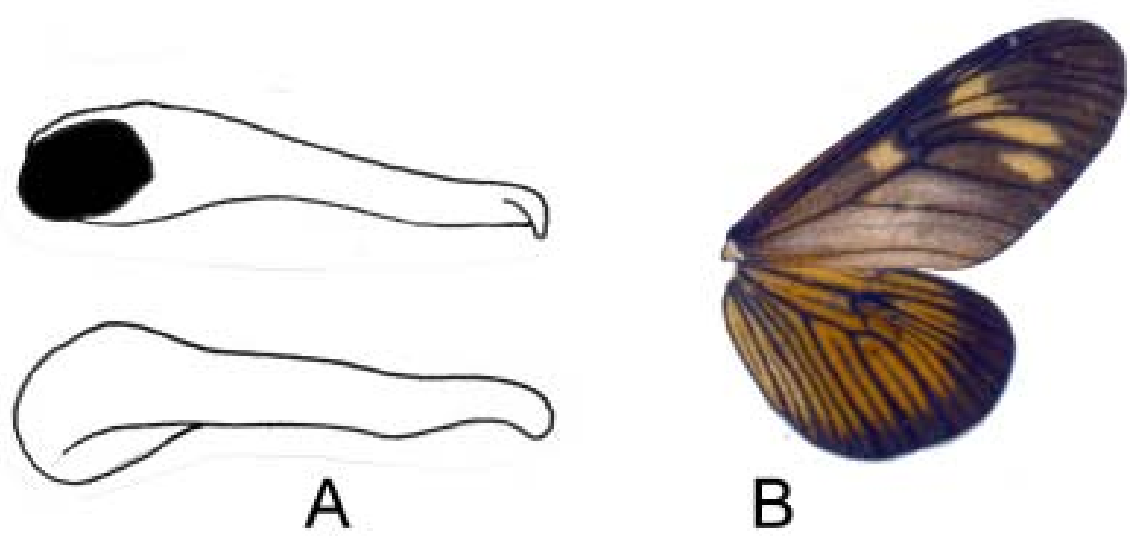

Figure 4. Actinote genitrix. (A) Dorsal and ventral views of the valva; (B) wings, dorsal surface.

6a. In ventral view, inner contour of valva bulging before apex, apex with a broad point (Figure 5A) discrepans

6b. In ventral view, inner contour of valva a simple arch, apex blunt (Figure 5B) eberti

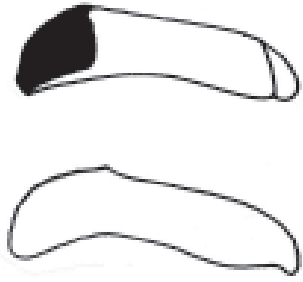

A
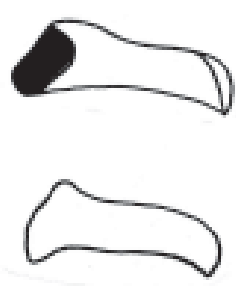

B

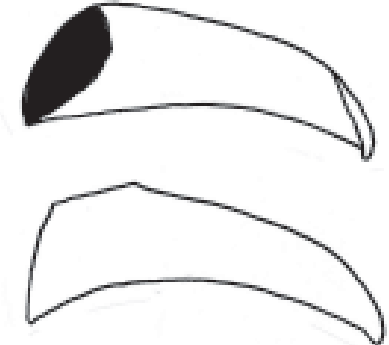

C

Figure 5. Dorsal (top) and ventral (bottom) views of the valva. (A) A. discrepans; (B) A. eberti; (C) A. melanisans.

7a. In ventral view, valva longer and somewhat arched (characters visible without dissection; Figure 6A) melanisans

7b. In ventral view, valvae shorter, less arched, with a slight constriction before the apex and a more sharply pointed apex (characters visible without dissection; Figure 6B). rhodope 

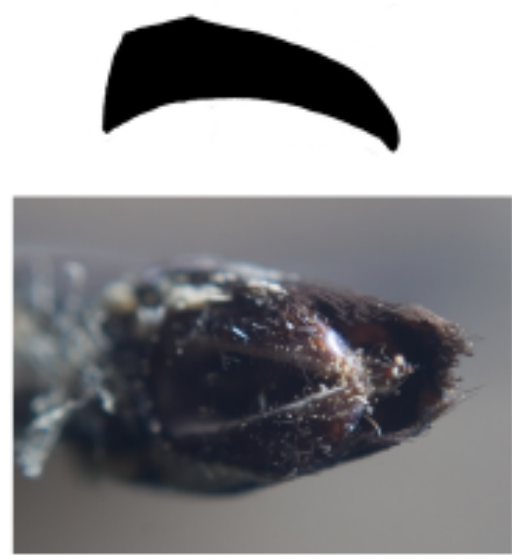

A
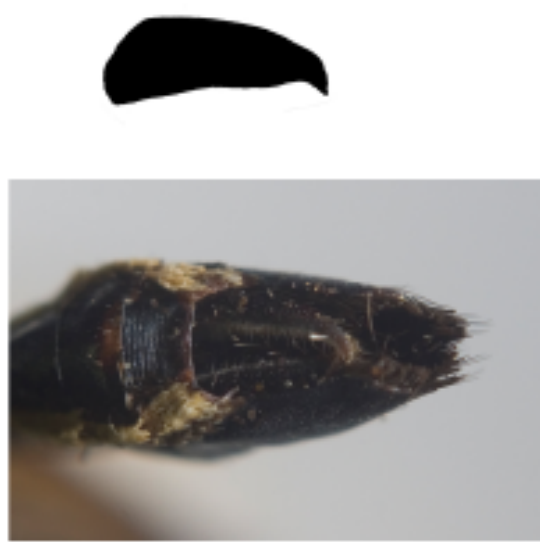

B

Figure 6. Ventral view of the valva and abdomen. (A) A. melanisans; (B) A. rhodope.

8a. In dorsal view, fore- and hindwing with orange stripes and spots, completely lacking yellow stripes or spots (example in Figure 7A).

8b. In dorsal view, fore- and hindwing stripes and spots predominantly orange, but some yellow stripes and spots present (example in Figure 7C)....

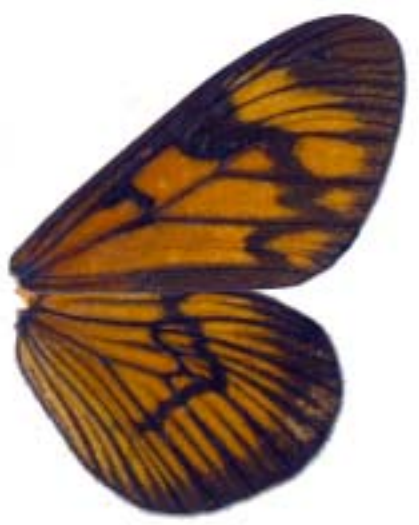

A

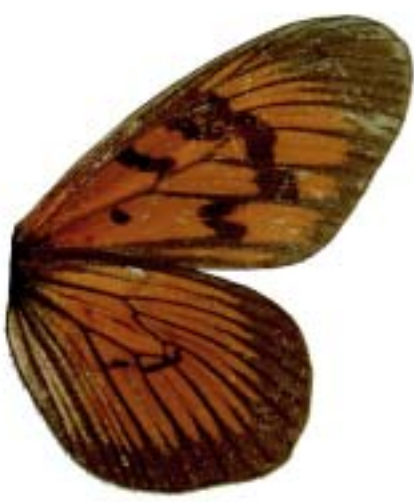

B

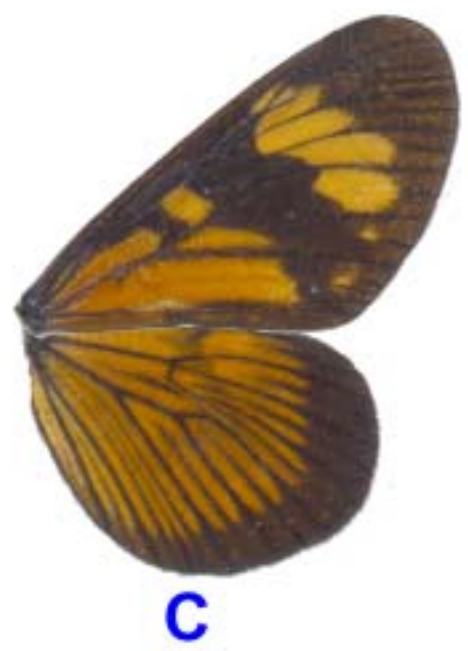

Figure 7. Dorsal view of fore- and hindwings. (A) A. surima; (B) A. catarina; (C) A. pyrrha.

9a. In dorsal view, wing background nearly black, markings dark orange, forewing stripes and spots never fused; in ventral view, valva short and with a sharply pointed apex (Figures 7A, 8A) ... surima

http://www.biotaneotropica.org.br 
9b. In dorsal view, wing background brown, markings dark orange, forewing stripes and spots often fused (common in the discocellular markings); in ventral view, valva elongated, broad at base and with a pointed apex (Figures 7B, 8B)

catarina
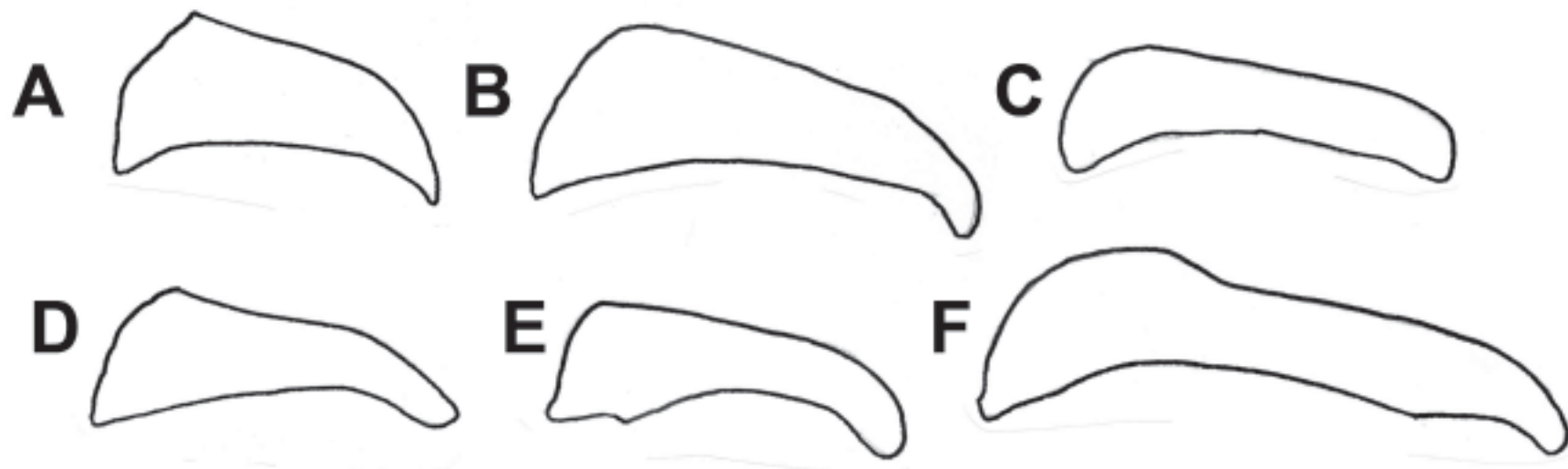

Figure 8. Ventral view of the valva. (A) A. surima; (B) A. catarina; (C) A. alalia; (D) A. bonita; (E) A. dalmeidai; (F) A. conspicua.

10a. Scales of the basal region of the hindwing with a prominent, broad mid-lobe - a pattern that differs from the typically serrate or dentate (Figure 9A) alalia

10b. Scales of the basal region of the hindwing dentate (examples in Figure 9B-C) 11

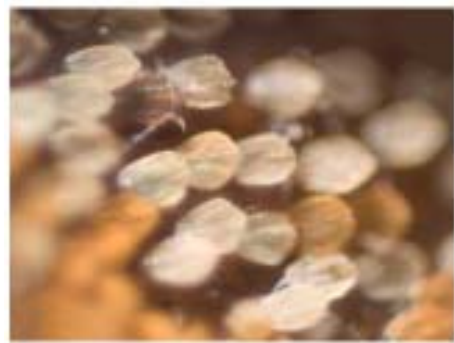

A

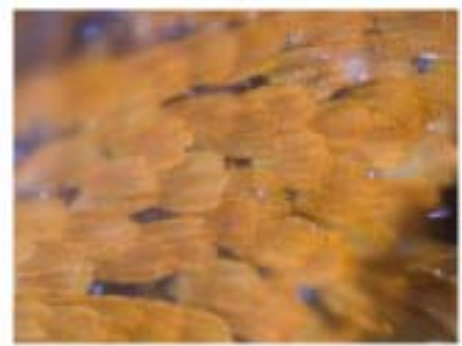

B

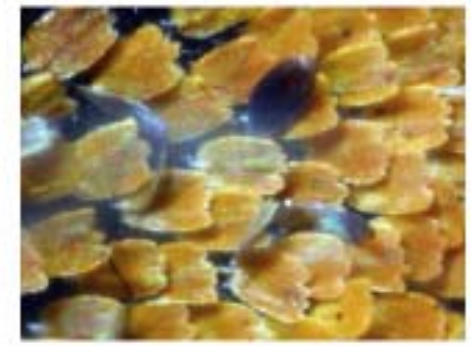

C

Figure 9. Scales of the basal region of the hindwing. (A) A. alalia; (B) A. conspicua; (C) A. bonita.

11a. Valva about $5 x$ longer than wide (maximum length / maximum width; Figure 10A) conspicua

11b. Valva 3x or less longer than wide (maximum length / maximum width; examples in Figure 10B-C) ..... 


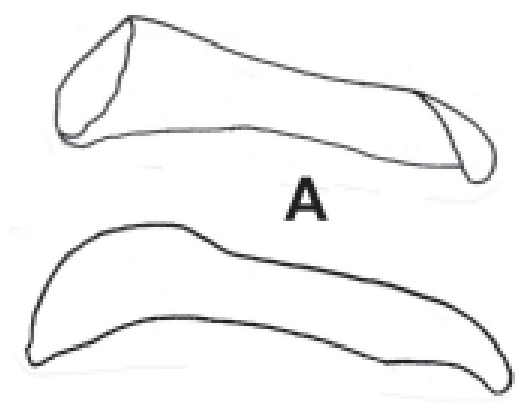

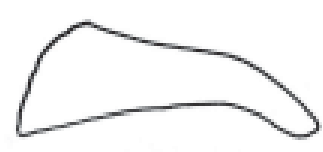

B

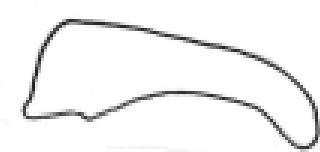

C

Figure 10. Valva. (A) A. conspicua, dorsal (top) and ventral (bottom) views; ventral view of (B) A. bonita; and (C) A. dalmeidai.

12a. In lateral view, valva sharply decrease in width from base to apex, apex narrower (Figure 11A) bonita

12b. In lateral view, valva with a slight decrease in width from base to apex, apex broader (Figure 11B) dalmeidai
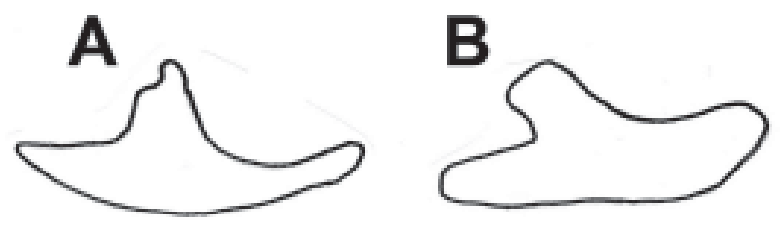

Figure 11. Lateral view of the valva; (A) A. bonita; (B) A. dalmeidai.

13a. Cell Cu2 with a thin black line across the yellow marks resembling an extra vein (blue arrow in Figure 12A); in ventral view, valvae strongly arched (Figure 12B) quadra

13b. Cell Cu2 without a thin black line across the yellow marks (example in Figure 12C); in ventral view, valvae not strongly arched (example in Figure 12D)

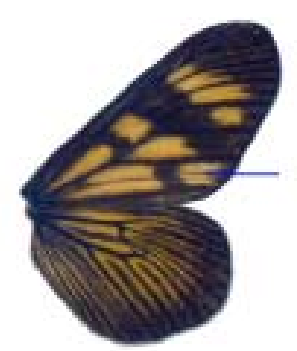

A

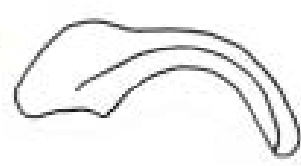

B

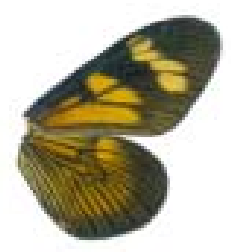

C

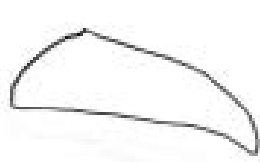

D

Figure 12. Dorsal view of the wings and ventral view of the valva. (A-B) A. quadra; (C-D) A. pellenea.

http://www.biotaneotropica.org.br 
14a. Hindwing with reddish-orange markings on both dorsal (Figure 13A) and ventral surfaces (Figure 13B) parapheles

14b. Hindwing without reddish-orange markings on dorsal and ventral surfaces 15
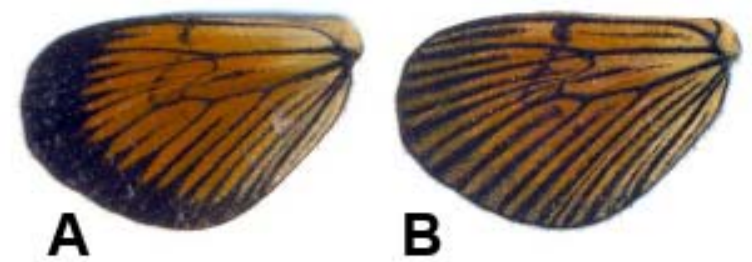

Figure 13. Dorsal (A) and ventral (B) views of the hindwing of A. parapheles.

15a. Scales of the basal region of the hindwing not dentate or serrate (examples in Figure 14A-B)

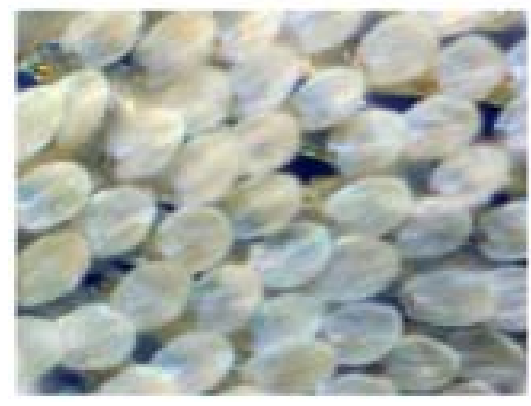

A

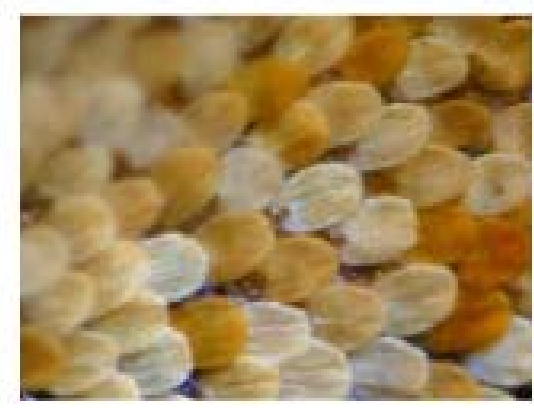

B

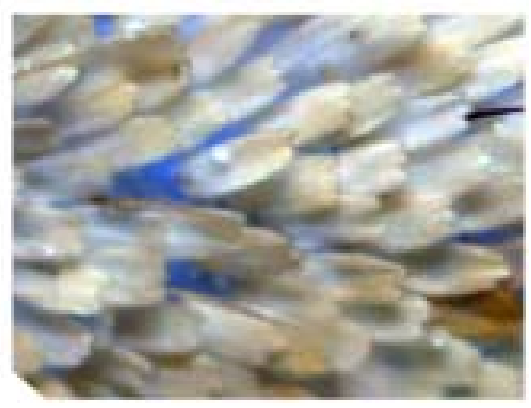

C

Figure 14. Scales of the basal region of the hindwing. (A) A. carycina; (B) A. pellenea; (C) A. pyrrha.

16a. Wing length generally smaller $(1.8-3.0 \mathrm{~cm}$, mean $=2.2 \mathrm{~cm})$; basal dorsal forewing markings velvety orange, varying from darker to paler between individuals; basal dorsal forewing markings generally broad, but varying in width between individuals (Figure 15 A-B); this species is very similar to carycina

16b. Wing length generally larger $(2.2-2.9 \mathrm{~cm}$, mean = $2.6 \mathrm{~cm})$; dorsal background color orangish-brown; basal dorsal forewing markings more shiny than pellenea, colored orange to yellow and white, sometimes the orange basal markings with a tint of white; basal dorsal forewing markings generally narrower than pellenea, but varying in width between individuals from broad to a faint diffuse line (Figure $15 \mathrm{C}$-D); this species is very similar to pellenea; some individuals may be similar to pyrra or pratensis carycina 

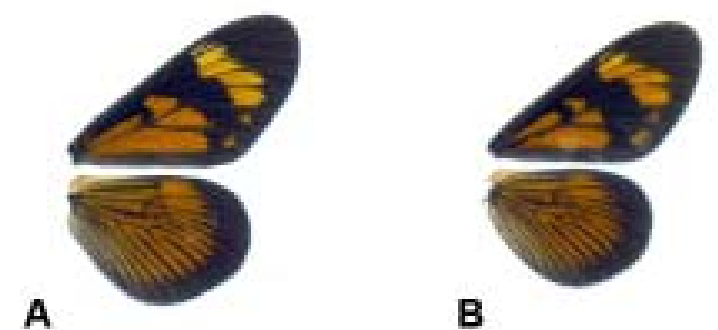

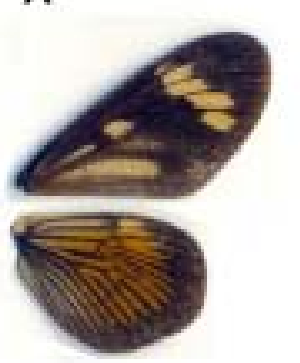

C
B

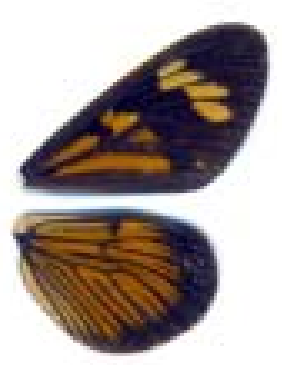

D

Figure 15. Dorsal (right) and ventral (left) views of wings. (A-B) A. pellenea; (C-D) A. carycina.

17a. In ventral view, valva apex blunt (blue arrow in Figure 16A)

pratensis

17b. In ventral view, valva apex more pointed (example in Figure 16B) 18
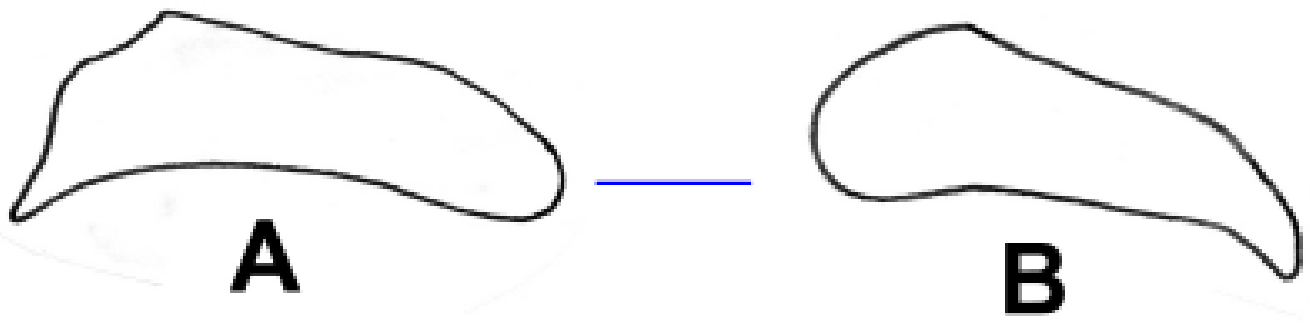

Figure 16. Ventral view of the valva. (A) A. pratensis; (B) A. brylla.

18a. In ventral view, inner and outer edges of valva nearly parallel (blue arrows in Figure 17A); dorsal forewing base markings predominantly shiny orange (Figure 16B) pyrrha

18b. In ventral view, inner and outer edges of valva converging toward apex (blue arrows in Figure 17C); dorsal forewing base markings predominantly yellow (Figure 17D)... brylla 

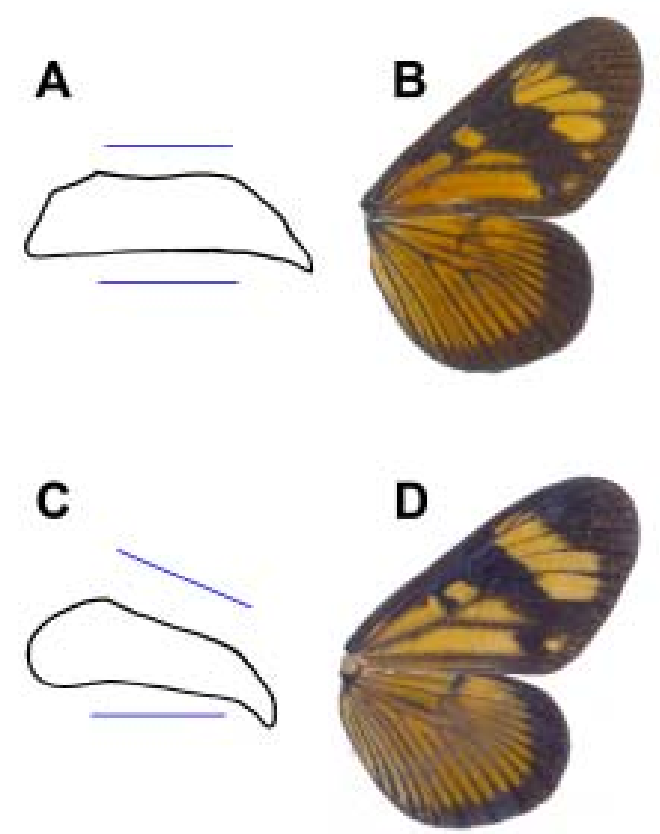

Figure 17. Ventral view of the valva and dorsal view of the wings.

(A-B) A. pyrrha; (C-D) A. brylla.

\section{Species accounts}

\section{Actinote morio Oberthür, 1917}

(Plate 1)

Actinote morio Oberthür 1917:112, 123; D’Almeida 1951:5; D’Abrera 1987:444; Brown 1988:36; Francini 1989:41, 53, 198; Brown 1992:158-159; Lamas 2004:263

\section{Actinote mario [sic] D’Abrera 1987:444}

Diagnosis: Wings opaque, iridescent black; dorsal basal forewing markings faint and diffuse, dirty yellow; post medial dorsal forewing markings dirty yellow; hindwing markings dirty yellow. Valva broad, slightly flattened dorso ventrally, somewhat uniform in width and lacking constrictions or terminal indentations; apex robust, pointed. Similar to, but distinguished from zikani by the darker wings and the shape of the valva.

Distribution: Brazil - Rio de Janeiro, Minas Gerais, Distrito Federal. Approximate altitude range: 700-1400 m.

Remarks: Iridescent-black mimicry group. Bivoltine. Relatively rare in collections.

\section{Actinote zikani D’Almeida, 1951}

(Plate 2)

Actinote zikani D’Almeida 1951:3-5; Lamas 1973:180; Mielke \& Casagrande 1986:151; Brown 1987:41; Francini 1989:41-43, 198; Fernandes, Machado \& Rylands 1990:60; Brown 1991:389; Lamas 2004:264

\section{Actinote mikani [sic] D’Almeida 1951:4 \\ Actinote zakani [sic] Brown 1988:36}

Actinote morio D’Almeida 1943:107-109

Diagnosis: Wings opaque, iridescent black; dorsal basal forewing markings faint and diffuse, dirty yellow; post medial dorsal forewing markings dirty yellow; hindwing markings dirty orange. Valva broad at the base, decreasing in width towards apex to produce a triangular shape in ventral view; apex robust, blunt. Similar to, but distinguished from morio by the lighter wing colors and the shape of the valva.

Distribution: Brazil - São Paulo, Rio de Janeiro. Approximate altitude range: $600-1200 \mathrm{~m}$.

Remarks: Iridescent-black mimicry group. Bivoltine. Relatively rare in collections.

\section{Actinote mamita (Burmeister, 1861)}

(Plate 3)

Acrea[sic] mamita Burmeister 1861:168

Actinote mamita Lewis 1975:43, 227; Penz 1983:5155; Penz 1985:51-53; Brown 1987:41; Brown 1988:36; Francini 1989:41, 43, 53-56, 61,63, 65, 67-71, 74-75, 77, 79, 87, 89-96, 100, 102, 107-109, 113-114, 135, 137, 140, 143, 153, 198, 199, 217, 220, 223; Brown 1992:158-159

Acraea mitama Schaus 1902:391

Actinote mamita mitama Eltringham \& Jordan 1913:12; Jordan 1913b:374; Jordan \& Eltringham 1916:19; Lamas 2004:263 
Diagnosis: Wings greasy, translucent; all forewing markings orange; hindwing comma-mark (Fig. 3A) always conspicuous. The subspecies from southeastern Brazil, mamita mitama (Schaus, 1902) seems to be more orange than mamita mamita found in Argentina. Valva cylindrical, somewhat narrow, and strongly arched dorso-ventrally. Distinguished from canutia by a larger marking on forewing cell Cu1, and all forewing markings being of the same color. Genitalia similar to canutia, but apex of valva more delicate in dorsal view.

Distribution: Brazil - Rio Grande do Sul, Santa Catarina, Paraná, São Paulo, Rio de Janeiro, Minas Gerais; Argentina; Uruguay?; Paraguay?. Approximate altitude range: 0-1600 m.

Remarks: Greasy-orange mimicry group. Lamas (2004) listed three subspecies of mamita (m. mamita, m. elena and m. mitama). We did not examine specimens of mamita elena (from Colombia; see Lamas 2004).

\section{Actinote canutia (Hopffer, 1874)}

(Plate 4)

Acraea canutia Hopffer 1874:345-346

Actinote canutia Jordan 1913b:374; Eltringham \& Jordan 1913:11; Jordan \& Eltringham 1916:19; Lewis 1975:227; Smart 1976:265; Pierre 1983:72; Brown 1987:41; D’Abrera 1987:445; Brown 1988:36; Francini 1989:41, 43, 53-56, 58, 61, 63, 65, 67-71, 74-75, 77, 79, 88-96, 98, 100, 102, 107-109, 113-114, 136-138, 140, 143, 146-147, 157-159, 165, 168, 191, 193, 198-199, 216-217, 220, 223; Brown 1992:158-159; Lamas 2004:263

Acraea (Actinote) canutia Pierre 1987:27

Actinote pellenea Geyer 1832: fig. 741-742 (part.)

Diagnosis: Wings greasy, translucent; basal forewing markings darker orange than postmedial markings; hindwing comma-mark faint (Fig. 3B). Valva cylindrical, somewhat narrow, and strongly arched dorso-ventrally. Distinguished from mamita by having a smaller marking on forewing cell Cu1, and by the post-medial forewing markings being lighter than basal markings. Genitalia similar to mamita, but apex of valva more robust in dorsal view.

Distribution: Brazil - Rio Grande do Sul?, Santa Catarina, São Paulo, Rio de Janeiro, Espírito Santo, Minas Gerais. Approximate altitude range: 0-1600 m.

Remarks: Greasy-orange mimicry group.

\section{Actinote genitrix D’Almeida, 1922}

(Plate 5)

Actinote genitrix D’Almeida 1922:143-145; D’Almeida 1925:335, 346-348, 352-353; ab. moesa D’Almeida 1925:348; ab. subrubicunda D’Almeida 1925:348; D’Almeida 1935a:71, 99-100, 102-103, 105, 109-110; var. a-f D’Almeida 1935a:99-
100; D’Almeida 1935b:487; Costa Lima 1936:136; D’Araujo e Silva et al. 1967:339; f. moesa Brown \& Mielke 1968:152; Toledo 1980:87; Penz 1983:37, 40; Penz 1985:104; Mielke \& Casagrande 1986:145-146, 148; ab. moesa Mielke \& Casagrande 1986:147, 149; ab. subrubicunda Mielke \& Casagrande 1986:147, 150; D’Abrera 1987:444; Brown 1987:41; Brown 1988:36; Francini 1989:41-43, 53, 55-56, 58, 61, 63, 65, 67-71, 74-75, 77-79, 87, 89-94, 100, 107-109, 114, 137-138, 140, 143, 157, 158-160, 165, 168, 191, 193, 198-199, 216-217, 220, 223; Brown 1992:158-159; Lamas 2004:263

\section{Actinote genitrix moesa D’Almeida 1935a:100}

Actinote genitrix subrubicunda D’Almeida 1935a:100

Actinote genetrix [sic] Ackery 1988:136

Diagnosis: Base of the forewings translucent, postmedial markings dirty white to creamy yellow. Valva very long (more than 5X longer than maximum width), broad at base and decreasing in width toward apex; straight for the most part, but with a small terminal arch. Similat to melanisans, rhodope, and discrepans (most often confused with melanisans). Forewings tend to be slightly more elongated at apex than in melanisans, rhodope, and discrepans. Easily distinguished from similar species by the length and shape of the valva.

Distribution: Brazil - Rio Grande do Sul, Santa Catarina, Paraná, São Paulo, Rio de Janeiro, Minas Gerais. Approximate altitude range: 600-1600 m.

Remarks: Black-yellow mimicry group.

\section{Actinote discrepans D’Almeida, 1958}

(Plate 6)

Actinote parapheles ab. discrepans D’Almeida 1935a:36-109; Mielke \& Casagrande 1986:151

Actinote discrepans D’Almeida 1958:2-4; Penz 1983:36, 38-39; Penz 1985:104; Brown 1987:41; Brown 1988:36; Francini 1989:41, 43, 53-56, 58, 61, 63, 65, 67-71, 74-75, 77, 79, 85, 89-96, 100, 107-109, 114, 117, 136-137, 140, 143, 153, 155 , 157-159, 194, 198, 217, 220, 223; Brown 1992:158-159; Lamas 2004:263

Diagnosis: Base of the forewings translucent, postmedial markings dirty white to creamy yellow. In fresh individuals, forewing discal markings often thin and sharp, resembling an exclamation point. Valva strongly arched dorsoventrally, with a slight constriction before apex in ventral view. Similat to genitrix, melanisans, and rhodope (most often confused with melanisans), but distinguished from these species by the sharp forewing discal markings and by the length and arched shape of the valva.

Distribution: Brazil - Paraná, São Paulo, Rio de Janeiro, Minas Gerais. Approximate altitude range: 600-1600 m.

Remarks: Black-yellow mimicry group. 


\section{Actinote eberti Francini, Freitas \& Penz, 2004}

(Plate 7)

Actinote eberti Francini, Freitas \& Penz 2004:2-5

Diagnosis: The wing color pattern of this species is intermediate between the darker morio and zikani (iridescent-black mimicry group) and the more colorful melanisans and rhodope (among other species in the black-yellow mimicry group). Wings with a greasy aspect, forewing translucent, colored areas faint but noticeable. Hindwing black margin broad, colored areas from faded orange to white. Males can be easily distinguished from similar species by the short arched valvae, broad tegumen, and small juxta.

Distrubution: Brazil - São Paulo, but possibly including Rio de Janeiro and Minas Gerais (along Serra da Mantiqueira). Approximate altitude: $1500 \mathrm{~m}$.

Remarks: Possibly included in the black-yellow mimicry group. This species is only known from nine collection specimens deposited in the Universidade Federal do Paraná.

\section{Actinote melanisans Oberthür, 1917}

(Plate 8)

Actinote bubona melanisans Oberthür 1917:124

Actinote melanisans D’Almeida 1958:1-2, 6-7; Ebert 1969:35; Araujo \& Valente 1981:1486; Penz 1985:104; Brown 1987:41; Brown 1988:36; Francini 1989:41, 43-44, 53-56, 58, 60, 62, 64, 67-71, 74-75, 77, 79, 84, 89-96, 99-103, 107-109, 113$114,117,136-138,140,141,143,146-147,152-153,155,157-$ 159, 161, 165-168, 187, 191, 193, 197-198, 215, 217, 220, 223; Lamas 2004:263

\section{Actinote cf. A. melanisans Penz 1983:32-35}

Actinote sp. B Penz 1983:38, 44, 45

Actinote brasiliensis var. melanisans D’Almeida 1925:339; ab. fuliginosa D’Almeida 1925:340; var. q D’Almeida 1935a:81; ab. fuliginosa D’Almeida 1935a:82; ab. fuliginosa Mielke \& Casagrande 1986:147, 150

Actinote carycina D’Abrera 1987:444

Actinote hyalina Brown 1992:158-159

Actinote morio Hayward 1931:26-27; Hayward 1935:96-97; D’Almeida 1935b:488; Hayward 1951:208

Actinote pyrrha D’Almeida 1935a:71, 86-87, 109-112; var. a-f D’Almeida 1935a:87-88; D’Almeida 1958:1-2, 4-5

Actinote rhodope Biezanko \& Freitas 1938:7 (part.); Biezanko \& Seta 1939:5 (part.); Biezanko, Ruffinelli \& Carbonell 1957:127 (part.); D’Araujo e Silva et al. 1968:340 (part.)

Actinote travassosi D’Almeida 1934:90-92

Diagnosis: Base of the forewings translucent, postmedial markings creamy yellow. Forewing discal and Cu2 markings generally faint, but discal markings may resemble those of discrepans in some individuals (i.e., shaped as an exclamation point). In ventral view, valva broad and some- what arched, and with a pointed apex. Similat to genitrix, discrepans, and rhodope, but distinguished from these species by the broad, arched and pointed valva.

Distribution: Brazil - Rio Grande do Sul, Santa Catarina, Paraná, São Paulo, Rio de Janeiro, Minas Gerais. Approximate altitude range: 0-1600 m.

Remarks: Black-yellow mimicry group. This is the most locally common species in this mimicry group. In the plates of Volume 5 of Seitz' Macrolepidoptera of the World, an individual that closely resembles melanisans and rhodope is illustrated and named hyalina. This illustration does not seem to correspond to any known type specimens designated by K. Jordan. In an attempt to clear this issue, Lamas (1996) designated a female lectotype (although the illustration was said to be male; Jordan 1913b) and downranked hyalina to a subspecies of $A$. pellenea. This seems to have further confused the matter, and the true identity of Jordan's hyalina may remain forever unknown.

\section{Actinote rhodope D’Almeida, 1923}

(Plate 9)

Actinote rhodope D’Almeida 1923:231-232; f. lynsa D’Almeida 1923:232; D’Almeida 1925:335-336, 342-345, 352-353; var. a-c D’Almeida 1925:345-346; ab. lynsa D’Almeida 1925:346; ab. tenuilimbata D'Almeida 1931:61; D’Almeida 1935a:71, 89-93, 103-105, 109-112; var. a-f D’Almeida 1935a:89-90; D’Almeida 1935b:487; Costa Lima 1936:136; Biezanko \& Freitas 1938:7 (part.); Biezanko \& Seta 1939:5 (part.); Biezanko, Ruffinelli \& Carbonell 1957:127; Brown \& Mielke 1968:152; D’Araujo Silva et al. 1968:340; Toledo 1980:87; Penz 1985:104; Mielke \& Casagrande 1986:147-148; f. lynsa Mielke \& Casagrande 1986:147, 149; ab. tenuilimbata Mielke \& Casagrande 1986:147, 149Brown 1987:41; Ackery 1988:136; Francini 1989:41-43, 53-56, 58, 60, 62, 64, 67-71, 74-75, 77, 78, 86, 89-96, 100, 107-108, 114, 136-138, 140, 143, 157, 158, 191, 193, 198, 217, 220, 223; Brown 1992:158159; Lamas 2004:264

Actinote hyalina D’Almeida 1922:143, 145

Actinote rhodope fuscata D’Almeida 1935a:90, 109; Mielke \& Casagrande 1986:151

Actinote rhodope lynsa D’Almeida 1935a:90, 109

Actinote rhodope tenuilimbata D'Almeida 1935a:90, 109

\section{Actinote melanisans Ebert 1969:35 (part.)}

Diagnosis: Base of the forewings translucent, postmedial markings creamy yellow. Forewing discal and Cu2 markings faint. In ventral view, valva short, somewhat broad and straight, and with a slight constriction anterior to the pointed apex. Similat to genitrix, discrepans, and melanisans (most often confused with melanisans); distinguished from these species by the shorter, straight and pointed valva. 
Distribution: Brazil - Rio Grande do Sul, Santa Catarina, Paraná, São Paulo, Rio de Janeiro, Minas Gerais. Approximate altitude range: $600-1600 \mathrm{~m}$.

Remarks: Black-yellow mimicry group. See also remarks for melanisans, above.

\section{Actinote surima (Schaus, 1902)}

(Plate 10)

Acraea surima Schaus 1902:392

Actinote surima Eltringham \& Jordan 1913:13; Jordan 1913b:374; Jordan \& Eltringham 1916:19; Hayward 1931:29, 169; Monte 1934:19; Hayward 1935:97; D’Almeida 1935a:94; D’Almeida 1935b:488; Biezanko \& Pitoñ 1941:9; Hayward 1951:209; f. punctata Hayward 1951:209; Biezanko, Ruffinelli \& Carbonell 1957:127; Brown \& Mielke 1967:95; Brown \& Mielke 1968:151; D’Araujo Silva et al. 1968:340; Ebert 1969:35; Brown \& Benson 1974:218; Smart 1976:191, 265; Penz 1983:46-50; Brown 1984:158; Penz 1985:109; Almeida, Souza \& Marques 1986:33; D’Abrera 1987:445; Brown 1987:41; Brown 1988:36; Francini 1989:44, 53-56, 58, 61, 74-75, 77, 79, 88-96, 100, 102, 107-109, 113-114, 136-137, 140, 143, 153, 155, 157-159, 165, 191, 193, 198-199, 217, 220, 223; Brown 1992:158-159; Paluch, Casagrande \& Mielke 1999:129-140; Lamas 2004:264

\section{Actinote surima punctata Hayward 1935:97}

Acraea (Actinote) surima Pierre 1987:22

Diagnosis: For the subspecies found in SE Brazil, surima surima: wings opaque, all markings dark orange, hidwing comma-mark well developed. Ventral hindwing uniform in color, and paler than dorsal hindwing. Valva short and broad, slightly compressed dorsoventrally, with a pointed apex.

Distribution: Brazil - Rio Grande do Sul, Santa Catarina, Paraná, São Paulo, Rio de Janeiro, Minas Gerais, Distrito Federal, Goiás; Paraguay; Argentina. Approximate altitude range: $0-1600 \mathrm{~m}$.

Remarks: Red mimicry group. Lamas (2004) listed two subspecies of surima (s. surima from Brazil and s. perisa from Argentina).

\section{Actinote catarina Penz, 1996}

(Plate 11)

Actinote catarina Penz \& Francini 1996:311-315; Lamas 2004:263

Actinote cf. A. alalia conspicua Penz 1983:56

Diagnosis: In dorsal view, all forewing markings orange. Forewing orange discal markings often fused, other orange spots commonly fused. In ventral view, hindwing pale yellow with a blurry orange stain at the center. Valvae broad, compressed dorso-ventrally and slightly arched laterally; apex pointed.
Distribution: Brazil - Rio Grande do Sul, Santa Catarina, Paraná. The accurate altitude range of this species is not known. The type specimens were collected inside one of the canyons in the southern end of the Serra Geral in Rio Grande do Sul (Faxinal, located near the city of Cambará do Sul, $1200 \mathrm{~m}$ ). We do not know how far these butterflies go down these canyons.

Remarks: Red mimicry group.

\section{Actinote alalia (C. Felder \& R. Felder, 1860)}

(Plate 12)

Acraea alalia C. Felder \& R. Felder 1860:105; Kirby 1871:136; Mabilde1896:64 (part.); var. Jones \& Moore 1883:36 (part.)

Acraea eulalia [sic] Müller 1878a:296 (part.)

Actinote alalia Jordan \& Eltringham 1916:19 (part.); D’Almeida 1935a:93 (part.); Ebert 1969:35; Lewis 1975:43, 227 (part.); Smart 1976:265 (part.); D’Abrera 1987:445; Ackery 1988:136; Lamas 2004:263

Actinote alalia alalia Eltringham \& Jordan 1913:10; Jordan 1913b:374 (part.)

Acraea (Actinote) alalia Pierre 1987:22 (part.)

Diagnosis: Wings opaque, all markings dark orange. Ventral hindwing paler than dorsal, ventral hindwing medial area darker orange than basal and post-medial areas. Valva cylindrical, moderately arched laterally and dorso-ventrally; in lateral view, similar in length to tegumen+uncus; apex robust and blunt. Similar to dalmeidai, but can be distinguished from this species by the longer and less laterally arched valva.

Distribution: Brazil - Rio Grande do Sul?, Santa Catarina, Paraná, São Paulo, Rio de Janeiro. Approximate altitude range: $1400-2000 \mathrm{~m}$.

Remarks: Red mimicry group.

\section{Actinote conspicua Jordan, 1913}

(Plate 13)

Actinote alalia conspicua Jordan 1913a:32; Jordan 1913b:374; Jordan \& Eltringham 1916:18; D’Abrera 1987:445

Actinote conspicua Brown \& Mielke 1968:151; Ebert 1969:35; Lamas 2004:263

Diagnosis: Large. Wings opaque, all markings dark orange. Ventral hindwing homogeneously dark orange. Valva cylindrical, long and narrow in lateral view, moderately arched dorso-ventrally; apex robust and blunt.

Distribution: Brazil - Paraná, São Paulo, Rio de Janeiro, Minas Gerais. Approximate altituderange: $1600-2000 \mathrm{~m}$.

Remarks: Red mimicry group. 


\section{Actinote bonita Penz, 1996}

(Plate 14)

Actinote bonita Penz \& Francini 1996:311-313, 315; Lamas 2004:263

Diagnosis: Wings opaque, all markings orange. Ventral hindwing pale orange, homogeneous. Valva cylindrical; in lateral view, broad at base and decreasing in diameter toward apex; moderately arched dorso-ventrally; in ventral view, slightly bent before apex; apex blunt. Can be easily distinguished from quadra by the color of the ventral hindwing and less arched valva.

Distribution: Brazil - Minas Gerais, Rio de Janeiro. Approximate altitude range: $1600-2000 \mathrm{~m}$.

Remarks: Red mimicry group.

\section{Actinote dalmeidai Francini, 1996}

(Plate 15)

Actinote dalmeidai Penz \& Francini 1996:310-313; Lamas 2004:263

Actinote alalia D’Almeida 1935a:71, 93 (part.); Brown 1987:41; Brown 1988:36; Francini 1989:41, 43, 53-56, 58, 61, 63, 65, 67-71, 74-75, 101, 107-109, 114, 135, 194, 198, 217, 220 , 223

\section{Actinote sp. near alalia Brown 1992:158-159}

Diagnosis: Wings opaque, all markings dark orange. Ventral hindwing paler than dorsal hindwing, ventral hindwing medial area darker orange than basal and postmedial areas. Valva cylindrical, moderately arched dorsoventrally, strongly arched laterally; in lateral view, valva shorter than tegumen+uncus; apex robust and blunt. Similar to, but distinguished from alalia by the shorter and more laterally arched valva.

Distribution: Brazil - Rio Grande do Sul, Santa Catarina, Paraná, São Paulo, Rio de Janeiro, Minas Gerais. Approximate altitude range: 600-1800 m.

Remarks: Red mimicry group.

\section{Actinote quadra (Schaus, 1902)}

(Plate 16)

Acraea quadra Schaus 1902:392

Actinote quadra Jordan 1913b:373; Eltringham \& Jordan 1913:13; Jordan \& Eltringham 1916:18; Lewis 1975:227; Smart 1976:265; D’Abrera 1987:445; Brown 1987:41; Brown 1988:36; Francini 1989:41, 53, 135, 198; Lamas 2004:263

\section{Acraea (Actinote) quadra Pierre 1987:22}

Diagnosis: Wings opaque, all markings orange. Ventral hindwing pale yellow, homogeneous. Valva cylindrical; strongly arched laterally; apex blunt. Similar to, but easily distinguished from bonita by the pale yellow ventral hindwing and the strongly arched valva.
Distribution: Brazil - São Paulo, Rio de Janeiro, Minas Gerais. Approximate altitude range: $1600-2000 \mathrm{~m}$.

Remarks: Orange-yellow mimicry group.

\section{Actinote parapheles Jordan, 1913}

(Plate 17)

Acraea thalia Müller 1882:263; Müller 1883b:216; Bönninghausen 1896:33

Actinote parapheles Eltringham \& Jordan 1913:12; Jordan 1913a:33; Jordan 1913b:373; f. pallescens Jordan 1913b:373; Jordan \& Eltringham 1916:18; D’Almeida 1925:335, 348-353; var. a-d D’Almeida 1925:351; var. fumida D'Almeida 1925:351; var. hippea D’Almeida 1925:354; Hayward 1931:2728, 167-168; f. ochreana Hayward 1931:28, 179-180; Hayward 1935:96-97; f. ochreana Hayward 1935:97; D’Almeida 1935a:71, 94-99, 103-105, 109-112; Brown 1987:41; Ackery 1988:136; Brown 1988:36; Francini 1989:41-43, 53-55, 56, 58, 63, 65, 67-71, 74-75, 77, 79, 86, 89, 90-96, 98, 100-102, 107-109, 113-114, 117, 135-138, 140-141, 143, 153, 157, 158-159, 165, 168, 191, 193, 197-199, 217, 220, 223; Brown 1992:158-159; Lamas 2004:263

Acraea (Actinote) parapheles Pierre 1987:22

Actinote alalia Mabilde 1896:64

Actinote distincta D’Almeida 1922:141-142, 145

Diagnosis: Wings opaque; basal markings orange, postmedial yellow. Ventral hindwing dark orange, homogeneous. Valva cylindrical; in ventral view, long and straight; in lateral view, moderately arched dorso-ventrally; apex blunt.

Distribution: Brazil - Rio Grande do Sul?, Santa Catarina, Paraná, São Paulo, Rio de Janeiro, Minas Gerais; Argentina; Paraguay; Uruguay?. Approximate altitude range: $0-1600 \mathrm{~m}$.

Remarks: Orange-yellow mimicry group.

\section{Actinote pellenea Hübner, [1821]}

(Plate 18)

Actinote pellenea Hübner 1820-1824 [1821]:18 fig. 14; Eltringham \& Jordan 1913:12; Müller 1915:366; D’Almeida 1922:130-135, 145; f. euterpe D’Almeida 1922:136; D'Almeida 1925:334, 336, 352-353; var. a-f D’Almeida 1925:337; var. euterpe D'Almeida 1925:336; Monte 1934:19; Ronna 1934:35; D’Almeida 1935a:71-72, 74-78, 103-105, 109-112; var. a-v D’Almeida 1935a:72-73; var. carycinoides D’Almeida 1935a:73; D’Almeida 1935b:487; Costa Lima 1936:362 (part.); Biezanko \& Freitas 1938:7; Costa Lima 1950:291; Brown \& Mielke 1967:96; D’Araujo Silva et al. 1968:339; Brown \& Mielke 1968:151; Brown \& Benson 1974:218; Lewis 1975:43, 227; Smart 1976:265; Toledo 1980:87; Penz 1983:23-26; D’Abrera 1984:223; var. carycinoides Mielke \& Casagrande 1986:149-150; Brown 1987:41; D’Abrera 1987:443; Ackery 1988:136; Brown 1988:36; Francini 1989:24, 26, 41-44, 53-56, 
58, 60, 62, 64, 67-71, 74-75, 77, 79-80, 89-97, 100-109, 112, 114, 118-122, 124-134, 136-153, 155-159, 162-168, 173-176, 180 182, 184-185, 187-192, 194-198, 200, 2002-203, 205-212, 215, 217, 219, 220, 222-223, 225

Actinote pellenia [sic] Ehrlich \& Ehrlich 1978:694

Actinote pellena [sic] Mabilde 1896:63

Acraea pellenea Kirby 1871:136; Hoppfer 1874:345 (part.); Capronnier 1874:24; Jones \& Moore 1883:36, 73; Bönninghausen 1896:33; Müller 1886:424

Acraea (Actinote) pellenea Pierre 1987:22

Actinote pellenea pellenea Toledo 1980:87; Eltringham \& Jordan 1913:12; Jordan 1913b:372; Jordan \& Eltringham 1916:17; Brown 1984:158; Brown 1992:158-159; Lamas 2004:263-264

Actinote pellenea nordestina D’Almeida 1935a:74; Cardoso 1949:429; Mielke \& Casagrande 1986:150

Actinote pellenea euterpe D’Almeida 1935a:74

Actinote anteas Monte 1934:199; D’Araujo Silva et al. 1968:339

Actinote auloeda Oberthür 1917:113-114, 123

Actinote brylla [lorida] Oberthür 1917:113, 123

Acraea euterpe Kirby 1871:137

Actinote thalia Pritwittz 1865:137; Joseph 1911:9-12 (part.)

Diagnosis: The SE Brazilian subspecies pellenea pellenea can be recognized by basal forewing markings velvety orange, varying in color from paler to darker orange but never white. Basal markings of the forewing generally broad, occupying most of the cell height. Actinote pellenea pellenea is very similar to carycina both in wing pattern and male genitalia. Nonetheless, pellenea and carycina have distinct larvae, which grants support to their status as separate species.

Distribution: Brazil - Rio Grande do Sul, Santa Catarina, Paraná, São Paulo, Rio de Janeiro, Minas Gerais, Espírito Santo, Distrito Federal, Goiás, Bahia; Paraguay; Argentina; Uruguay. Approximate altitude range: 0-1200 m.

Remarks: orange-yellow mimicry group. Lamas (2004) listed 15 described and five undescribed subspecies for pellenea, of which pellenea pellenea is the only subspecies found in Brazil. Given the remarkable color variation and nondescript genitalic morphology of this species, a reassessment of the subspecies of pellenea is desirable.

Actinote carycina Jordan, 1913

(Plate 19)

Actinote carycina Jordan 1913b:373; Jordan \& Eltringham 1916:18; f. calchaqui Hayward 1931:20-22, 175177; D’Almeida 1935a:78-79, 109-112; var. a-l D’Almeida 1935a:79-80; var. hoffmani D’Almeida 1935a:79-80, 109; var. differens D’Almeida 1935a:80, 109; f. calchaqui D’Almeida 1935b:487; f. calchaqui Hayward 1935:95, 97; Costa Lima 1936:136; Biezanko \& Pitoñ 1941:9; Biezanko \& Ruffinelli 1957:36; Biezanko, Ruffinelli \& Carbonell 1957:127; Ebert 1969:35; Brown \& Benson 1974:218; Smart 1976:265; Penz 1983:14-22; Brown 1984:158; Penz 1985:104; Francini 1985:105; Almeida, Souza \& Marques 1986:33; D’Abrera 1987:444; Francini 1989:5, 12, 36, 41-44, 53-56, 58, 60, 62, 64, 68-71, 74-75, 77-79, 81, 89-97, 100-102, 107-109, 113-114, 118, 135-138, 140, 143, 146-147, 152-153, 155-159, 165, 167-168, 186-188, 190-192, 197-198, 200, 213, 215-217, 220, 223; var. hoffmani Mielke \& Casagrande 1986:148, 150; var. differens Mielke \& Casagrande 1986:150; Brown 1992:158-159; Paluch, Casagrande \& Mielke 2001:883-896; Lamas 2004:263

\section{Acraea (Actinote) carycina Pierre 1987:22}

Actinote calchaqui Toledo 1980:88-89

Actinote pellenea Hayward 1951:207

Actinote pellenea pellenea Orfila 1964:41-48

Actinote pyrrha Biezanko \& Freitas 1938:7 (part.)

Acraea thalia Müller 1876:3, 5, 8-10; Müller 1877a:214221; Müller 1877b:218-224; Burmeister 1878:126-127; var. ab Burmeister 1878:127; Müller 1878a:296; Müller 1878b:5455; Burmeister 1879:194; Müller 1879:2; Müller 1879c:106107 (part.); Gosse 1880:197 (part.); Müller 1881-82:263 (part.); Müller 1883a:415-416; Müller 1883b:216 (part.); Müller 1883c:197-201; Mabilde 1896:64 (part.)

Actinote thalia Joseph 1911:9-18 (part.); Müller 1915:186, 191-193, 195, 325, 340, 365, 366, 377, 380, 429 (part.); Monte 1934:19; D’Araujo Silva et al. 1968:339340; Ackery 1988:136

Diagnosis: Dorsal wing background color orangishbrown, basal forewing markings vary from orange to white, and from broad to narrow. In some specimens the orange basal markings have a tint of white. Very similar to pellenea, but despite the variation in wing color, it can generally be distinguished from pellenea by the narrower forewing basal markings and slightly larger wing size (the wing size range overlaps with that of pellenea, however). Some individuals may also have similar wing patterns to pratensis, but can be distinguished from this species by the genitalia. Genitalia very similar to pellenea. Actinote carycina and pellenea have distinct larvae, which grants support to their status as separate species.

Distribution: Brazil - Rio Grande do Sul, Santa Catarina, Paraná, São Paulo, Minas Gerais, Rio de Janeiro. Approximate altitude range: 0-1400 m.

Remarks: orange-yellow mimicry group. 


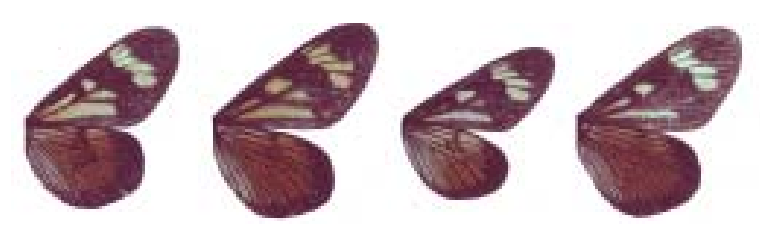

Figure 18. Variation in wing color and markings in male A. carycina from a single sib group.

\section{Actinote pratensis Francini, Freitas \& Penz, 2004}

(Plate 20)

Actinote pratensis Francini, Freitas \& Penz 2004:5-7

Diagnosis: Similar to pyrrha and carycina. Can be distinguished from these two and other species by the following combination of characters. Although their size range overlaps, in general male pratensis are larger than those of pyrrha and carycina. Basal markings of forewing orange, and those in cells $\mathrm{Cu}_{1}$ and $\mathrm{Cu}_{2}$ usually diffuse at the edges; sub-apical forewing markings dirty white, usually ranging from $\mathrm{R}_{5}$ to $\mathrm{Cu}_{1}$. PD spot absent. The dark border of hindwing is generally broader in pratensis than in pyrrha, but the ranges of variation in this character fully overlap between these two species. Male valva bowed, nearly homogeneous in width in lateral view, with blunt apex. Aedeagus shorter than the length of the genitalic capsule and comparatively thicker than those of pyrrha and carycina.

Distribution: Brazil - São Paulo, but possibly expanding to Minas Gerais (populations of pratensis were recorded in Águas da Prata, Rio Claro, Campinas and Jundiaí, all in São Paulo state). The altitude range for this species in unknown, but the known populations occur at ca. 600-800 m.

Remarks: Orange-yellow mimicry group.

\section{Actinote pyrrha (Fabricius, 1775)}

(Plate 21)

Papilio pyrrha Fabricius 1775:464; Goeze 1779:107; Fabricius 1781-1782:33; Fabricius 1787:16; Gmellin 1790:2252; Fabricius 1793:176; Gabriel 1927:102

Actinote pyrrha Eltringham \& Jordan 1913:13 (part.); Jordan 1913b:373; Jordan \& Eltringham 1916:18; Hayward 1931:25-26; Hayward 1935:96-97; f. illimnis Hayward 1935:9697; D’Almeida 1935b:488; Costa Lima 1936:136; Biezanko 1938:6; Biezanko \& Freitas 1938:7 (part.); Biezanko 1939:39; Biezanko \& Pitoñ 1941:9; Hayward 1951:209; Biezanko, Ruffinelli \& Carbonell 1957:127; D’Almeida 1958:1-3, 5; Brown \& Mielke 1967:96; Brown \& Mielke 1968:151;
D’Araujo Silva et al. 1968:339; Ebert 1969:35; Brown \& Mielke 1972:28-29 (part.); Brown \& Benson 1974:218; Smart 1976:265; Penz 1983:27-31; Brown 1984:158; Penz 1985:104; D’Abrera 1987:444; Brown 1987:41; Ackery 1988:136; Brown 1988:36; Francini 1989:41, 42-43, 53-56, 58, 60, 62, 64, 67-71, 74-75, 77, 79-80, 89-97, 100-102, 107-109, 113-114, 117, 136, 137-138, 141, 143, 146-147, 153, 155, 157-159, 168, 190-192, 197-198, 214-215, 217, 220, 223

\section{Acraea (Actinote) pyrrha Pierre 1987:22}

Actinote pyrrha illimnis Hayward 1951:209

Acraea anteas Gosse 1880:197; Staudinger 18841888:81; var. Müller 1886:426; Bönninghausen 1896:33

Actinote anteas Costa Lima 1936:136

Actinote brasiliensis D’Almeida 1922:136-139, 145; f. pyrrha D’Almeida 1922:140; var. b D’Almeida 1922:140; D’Almeida 1925:334-335, 338-339, 341-342, 352, 353; var. a-c D’Almeida 1925:340; D’Almeida 1935a:71, 80, 83-86, 103, 105, 109-112; var. a-z and aa D’Almeida 1935a:80; var. discolora D’Almeida 1935a:81; D’Almeida 1935b:487; Costa Lima 1936:136; Biezanko \& Freitas 1938:7; D’Araujo Silva et al. 1968:339; Toledo 1980:87; Mielke \& Casagrande 1986:145, 148; var. discolora Mielke \& Casagrande 1986:149, 151

Actinote bubona Oberthür 1917:117-118, 124; D’Abrera 1987:444

Actinote diaguita Toledo 1980:87-93

Acraea euterpe C. Felder 1862:475

Acraea pellenea Gosse 1880:197; Costa Lima 1950:291; f. diaguita Hayward 1935:95, 97; f. diaguita D’Almeida 1935b:488; f. diaguita Hayward 1951:208; f. diaguita Hayward 1968:350

Actinote pellenea diaguita Hayward 1931:2223, 177-178

Acraea thalia Aurivilius 1882:47 (part.); Godart 18191824:240; Lucas 1835:101 (part.); Butler 1870:128 (part.); Kirby 1871:135(part.); Capronnier 1874:24; Müller 1878a:296 (part.); Burmeister 1878:126-127 (part.); Gosse 1880:197; Müller 1883a:415; Staudinger 1884-1888:81; Haase 1893:54 
Actinote thalia Weymer 1894:321; Joseph 1911:9-18

Actinote thalia pyrrha Brown 1992:158-159; Lamas 2004:264

Diagnosis: Dorsal forewing basal markings generally broad, occupying the entire height of the cells, and predominantly shiny orange. In ventral view, inner and outer edges of valva nearly parallel.

Distribution: Brazil-Rio Grande do Sul, Santa Catarina, Paraná, São Paulo, Rio de Janeiro, Minas Gerais; Paraguay; Argentina. Approximate altitude range: 0-1600 m.

Remarks: Orange-yellow mimicry group. Brown (1992) and Lamas (1996, 2004) considered pyrrha a subspecies of Actinote thalia-the type species of Actinote (Hemming 1967). We maintain the species status and name pyrrha for two reasons. First, no characters or justification have been provided to unite the types of thalia (a male from "Indiis" = South America, probably Surinam) and pyrrha (a worn female from 'Brazil'), and it is thus unclear why these two taxa were considered conspecific by Brown (1992) and Lamas (1996, 2004). Second, inasmuch as the name pyrrha has been traditionally used for the taxon portrayed here, we conservatively consider Actinote pyrrha as separate species. We note, however, that a comparative study of both adult and larval morphology is needed to confirm the taxonomic status of pyrrha and verify the proposed subspecific classification of thalia.

\section{Actinote brylla Oberthür, 1917}

(Plate 22) 2004:263

Actinote brylla Oberthür 1917:116-117, 124; Lamas

Actinote brasiliensis var. j, p, a-c D’Almeida 1935a:81; var. jordani D’Almeida 1935a:82, 109; var. notabilis D’Almeida 1935a:82, 109-110; var. notabilis Mielke \& Casagrande 1986:151

Actinote jordani D’Almeida 1922:141, 145; D’Almeida 1958:4; Mielke \& Casagrande 1986:145, 148

Actinote notabilis D’Almeida 1958:4; Brown 1988:36; Francini 1989: 41, 43, 53-56, 58, 60, 62, 64, 67-71, 74-77, 79, 83, 89-97, 100, 102-104, 106-109, 112, 114, 119-120, 123-125, 132, $134,136-138,140,143,146-147,153,155,157,159,168,191-$ 192, 198, 217, 220, 223

Diagnosis: Male forewing length $2.8-3.4 \mathrm{~cm}$ (mean=3.1 cm). Dorsal forewing base markings predominantly yellow (predominantly orange in pyrrha). Hindwing comma-shaped mark always present. In ventral view, inner and outer edges of valva converging toward apex (Fig. 17C).

Distribution: Brazil - São Paulo, Rio de Janeiro, Espírito Santo, Bahia. Approximate altitude range: 0-500 m.

Remarks: Orange-yellow mimicry group. Actinote brylla can only be found below $500 \mathrm{~m}$ altitude in latitudes above $22^{\circ} \mathrm{S}$ (although it is possible that it exists in higher altitudes in Espírito Santo state). This species appears to be closely related to, and rarely sympatric with pyrrha. D’Almeida (1922a, 1935a) initially used two names for this species, later recognizing it as distinct under the name notabilis (D’Almeida 1958). Figures by Oberthür (1917), however, allow accurate identification of brylla, and were probably overlooked by D’Almeida.

\section{Concluding remarks}

All Actinote species of southeastern Brazil show intra-specific variation in wing color pattern so that, in some cases, there is a high degree of overlap between species. This variation can make species identification difficult, and we used sib groups to document some of the ranges of color and size of markings on the wings of several species (Fig. 15). Examples of species that overlap in both their geographical distributions and wing patterns are: (1) melanisans, genitrix, discrepans and rhodope; (2) alalia and dalmeidai; (3) carycina, pellenea, pyrrha and pratensis. All of these species, however, can be distinguished from one another by the male valva, often without dissection (e.g., Fig. 6 in the key, above). From experience, we note that having large series of specimens greatly facilitates identification.

Examination of large series of specimens indicates that, in addition to wing color variation, genital morphology can also vary in all Actinote species in southeastern Brazil. The parts that showed strongest variation were the saccus and tegumen+uncus, and some variation was also found in the phallobase (unpublished obs.). Variation in the valva morphology and proportions (length/width) was smaller, and this part of the male genitalia provided reliable characters for species identification.

Female identification still remains a challenge for many Actinote species. Similarly to what was noted here for males, rearing of sib-groups and examination of large series of specimens will help our understanding of female wing pattern variation and the discovery of diagnostic characters. Furthermore, examination of the sphragis and sterigma (e.g., Penz \& Francini 1996) will bring useful characters for identification of specimens in the field and collections. Future work will hopefully provide a key for female identification.

It is likely that more species of Actinote will be found in southeastern Brazil. Because some species are nearly indistinguishable, collections may have mixed series identified under the name of the most common species (e.g., pyrrha and pratensis). Furthermore, many areas have not been sampled systematically, where collectors might have missed the short flight period characteristic of Actinote. We hope that this key will be of help in the identification of Actinote butterflies and facilitate the finding and description of species that have gone unnoticed to us and to our predecessors. 


\section{Plate 1}

\section{Actinote morio}
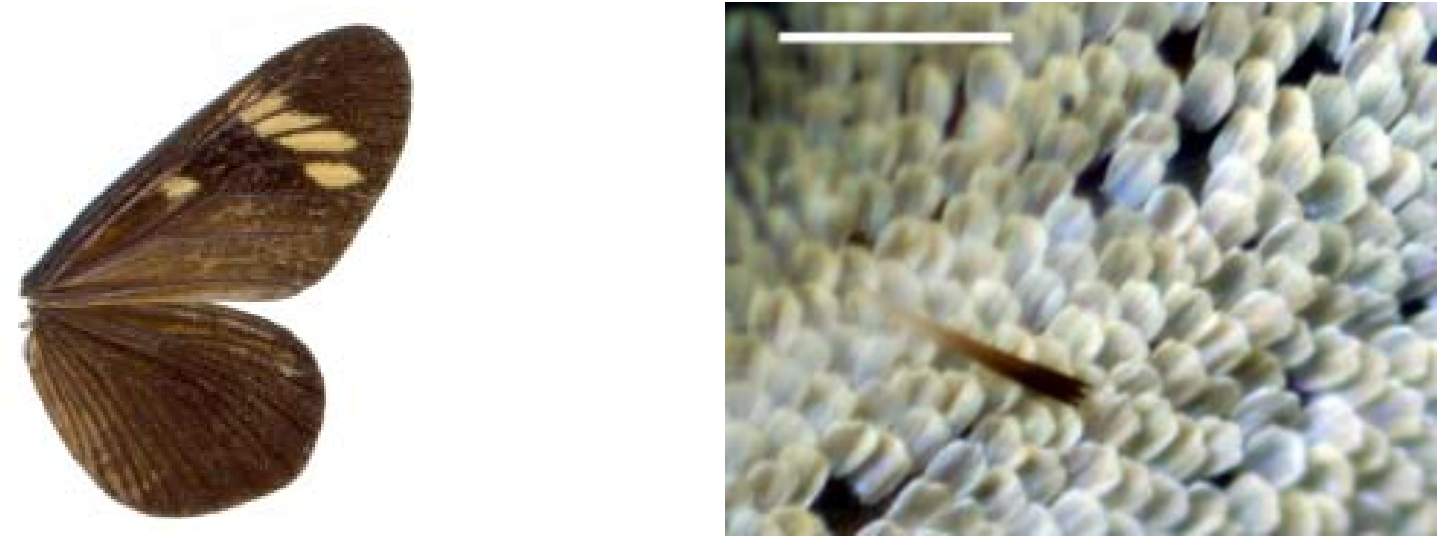

Wings in dorsal view

Scales of the basal area of the hindwing

Scale bar $0.1 \mathrm{~mm}$

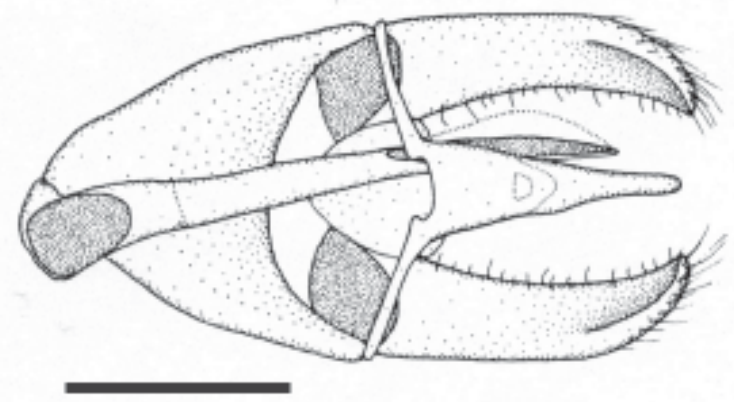

Genitalia in dorsal view

Scale bar $1 \mathrm{~mm}$

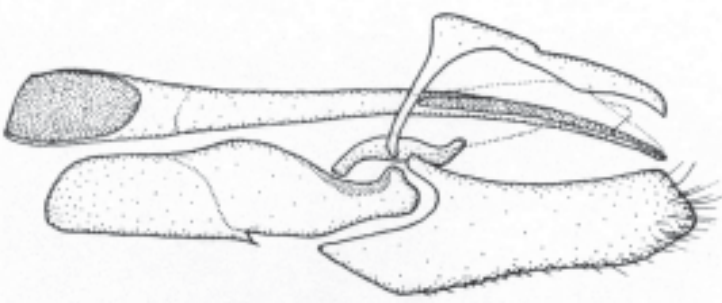

Genitalia in lateral view

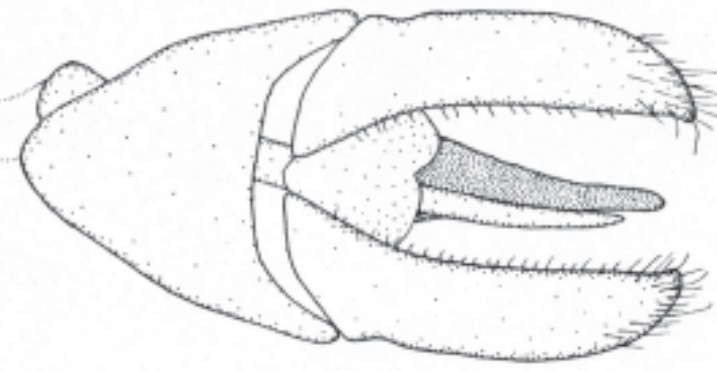

Genitalia in ventral view

http://www.biotaneotropica.org.br 
Plate 2

\section{Actinote zikani}

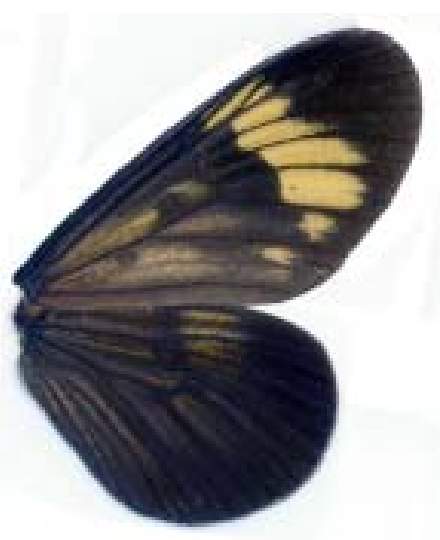

Wings in dorsal view
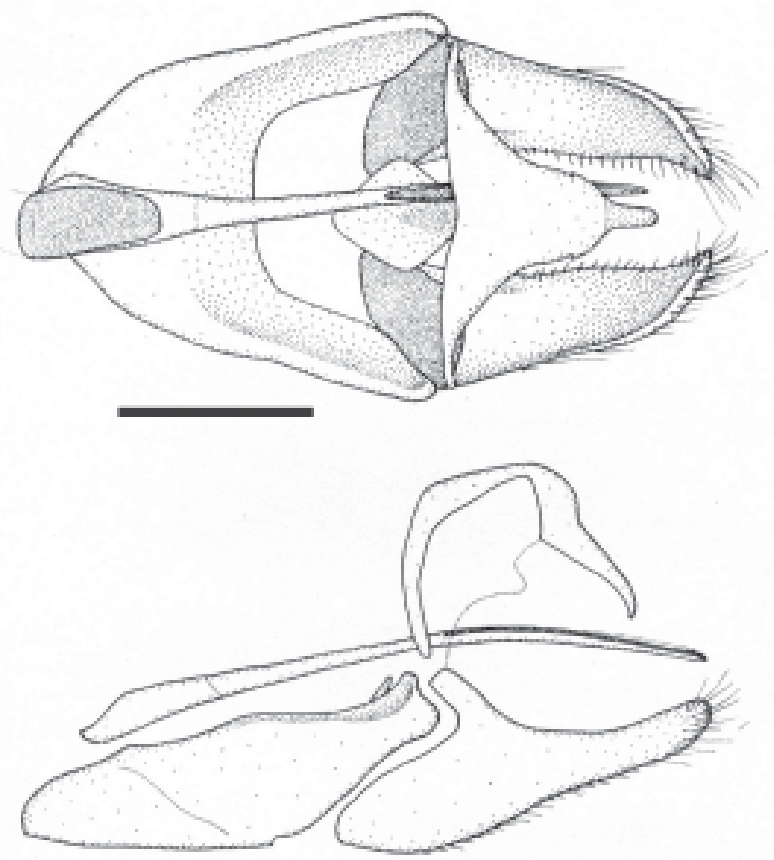

Genitalia in dorsal view Scale bar $1 \mathrm{~mm}$ Scale bar $0.1 \mathrm{~mm}$

\section{Scale bar 1 ming}

Genitalia in lateral view

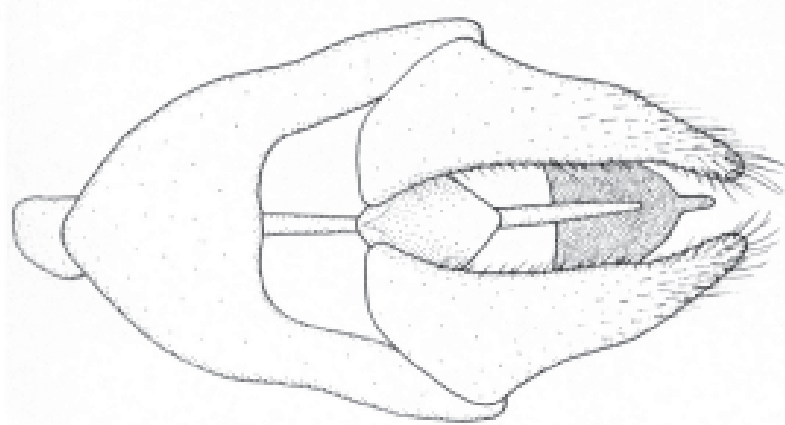

Scales of the basal area of the hindwing

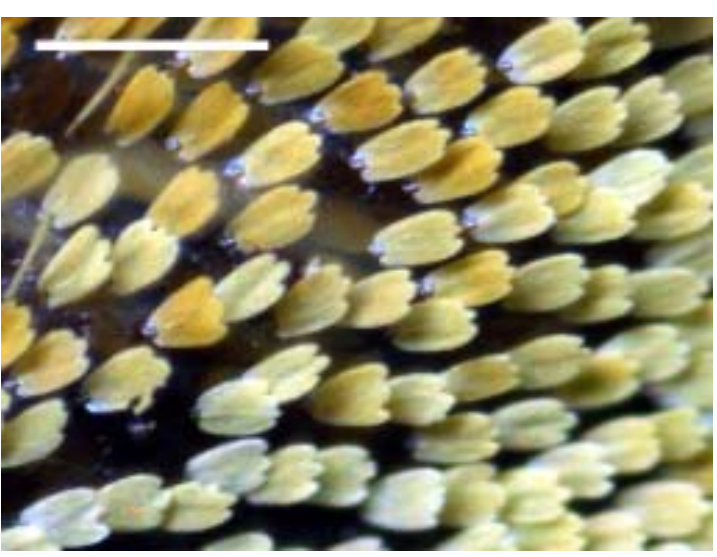

Genitalia in ventral view

http://www.biotaneotropica.org.br 


\section{Plate 3}

\section{Actinote mamita}

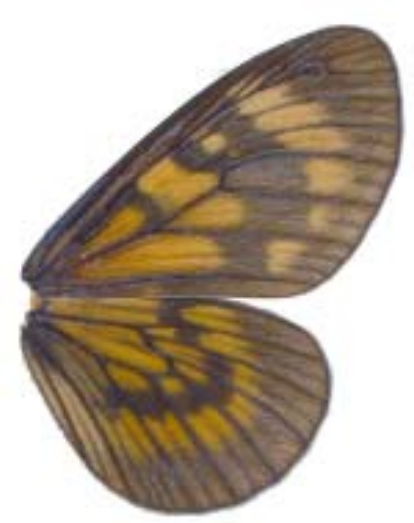

Wings in dorsal view
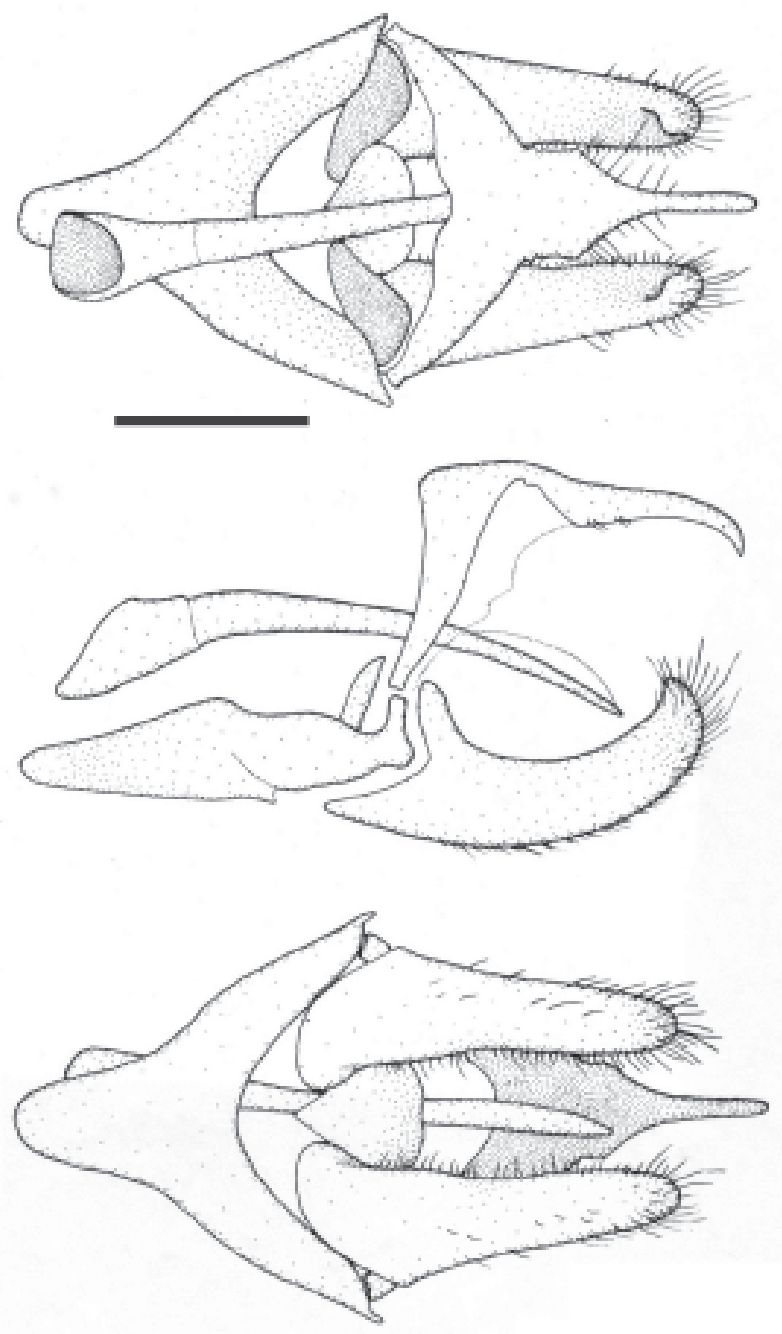

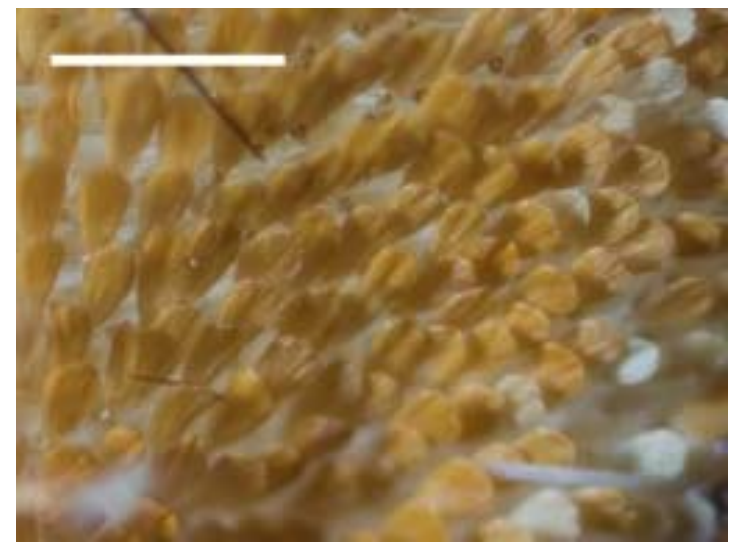

Scales of the basal area of the hindwing Scale bar $0.1 \mathrm{~mm}$

Genitalia in dorsal view Scale bar $1 \mathrm{~mm}$

Genitalia in lateral view

Genitalia in ventral view

http://www.biotaneotropica.org.br 


\section{Plate 4}

\section{Actinote canutia}

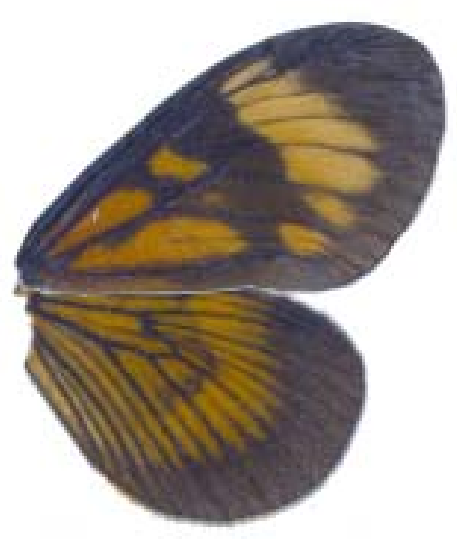

Wings in dorsal view
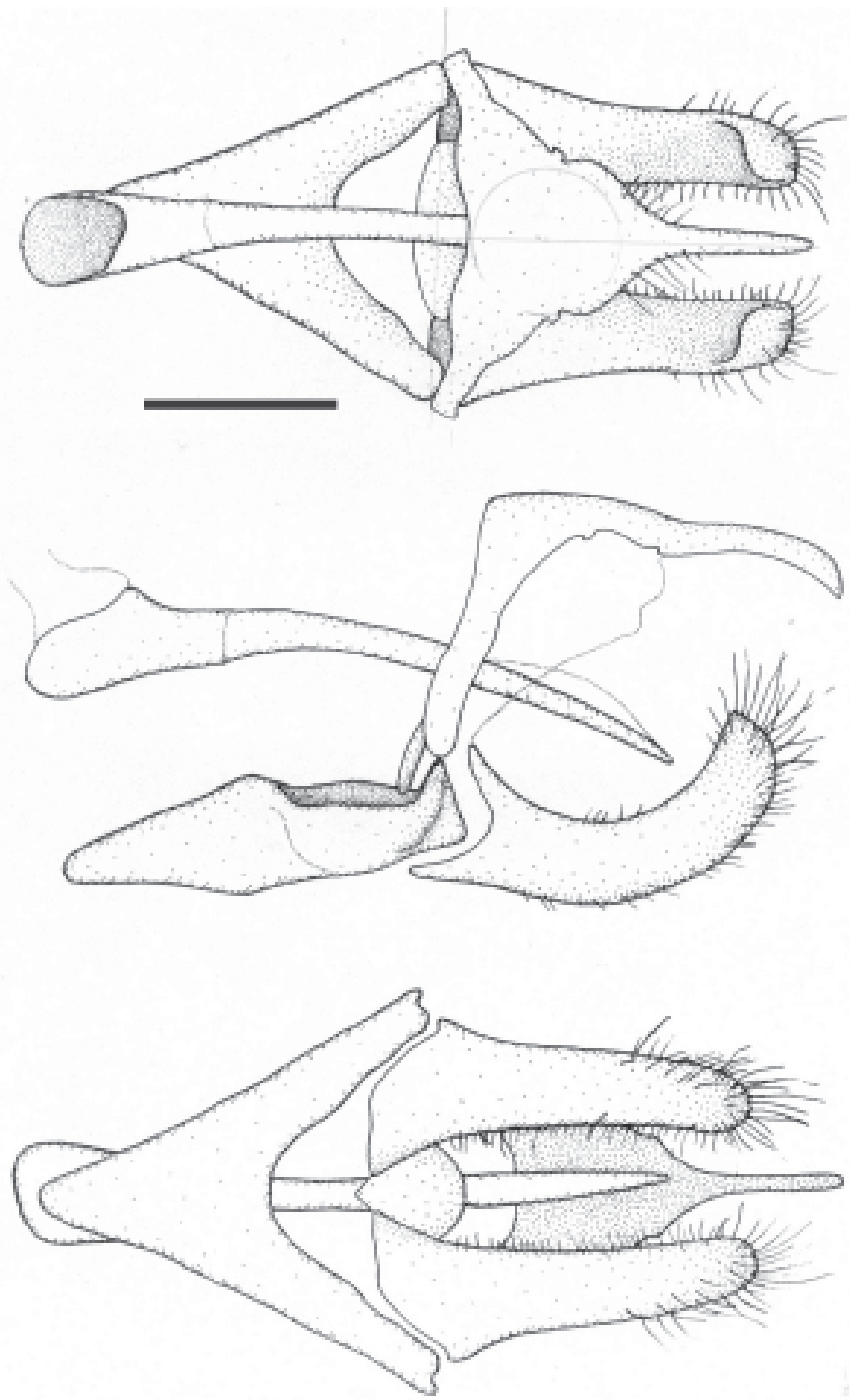

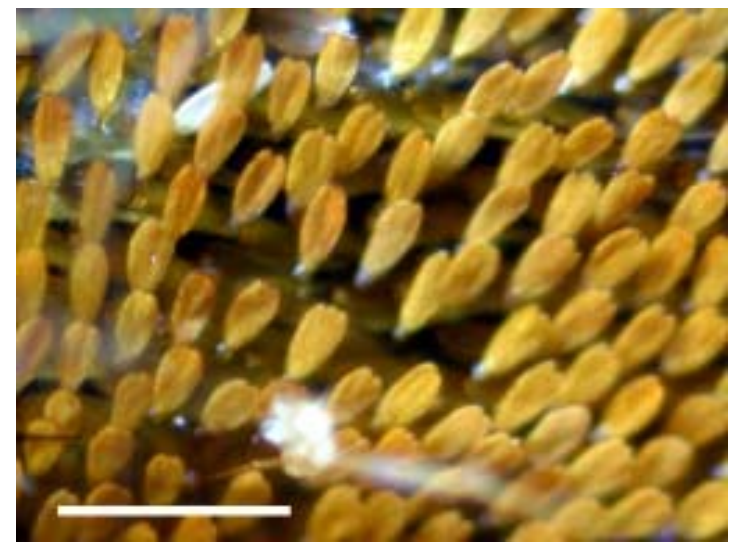

Scales of the basal area of the hindwing Scale bar $0.1 \mathrm{~mm}$

Genitalia in dorsal view

Scale bar $1 \mathrm{~mm}$

Genitalia in lateral view

Genitalia in ventral view 
Plate 5

\section{Actinote genitrix}

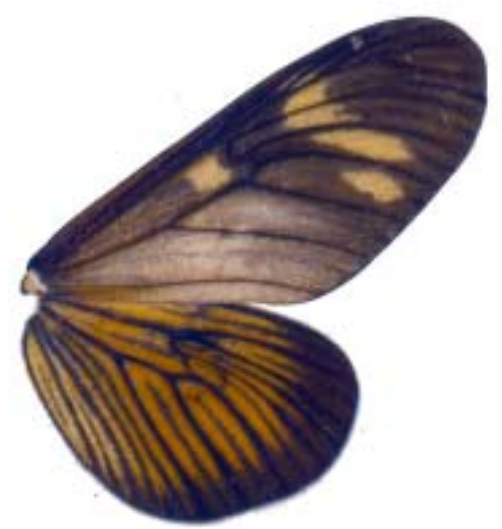

Wings in dorsal view
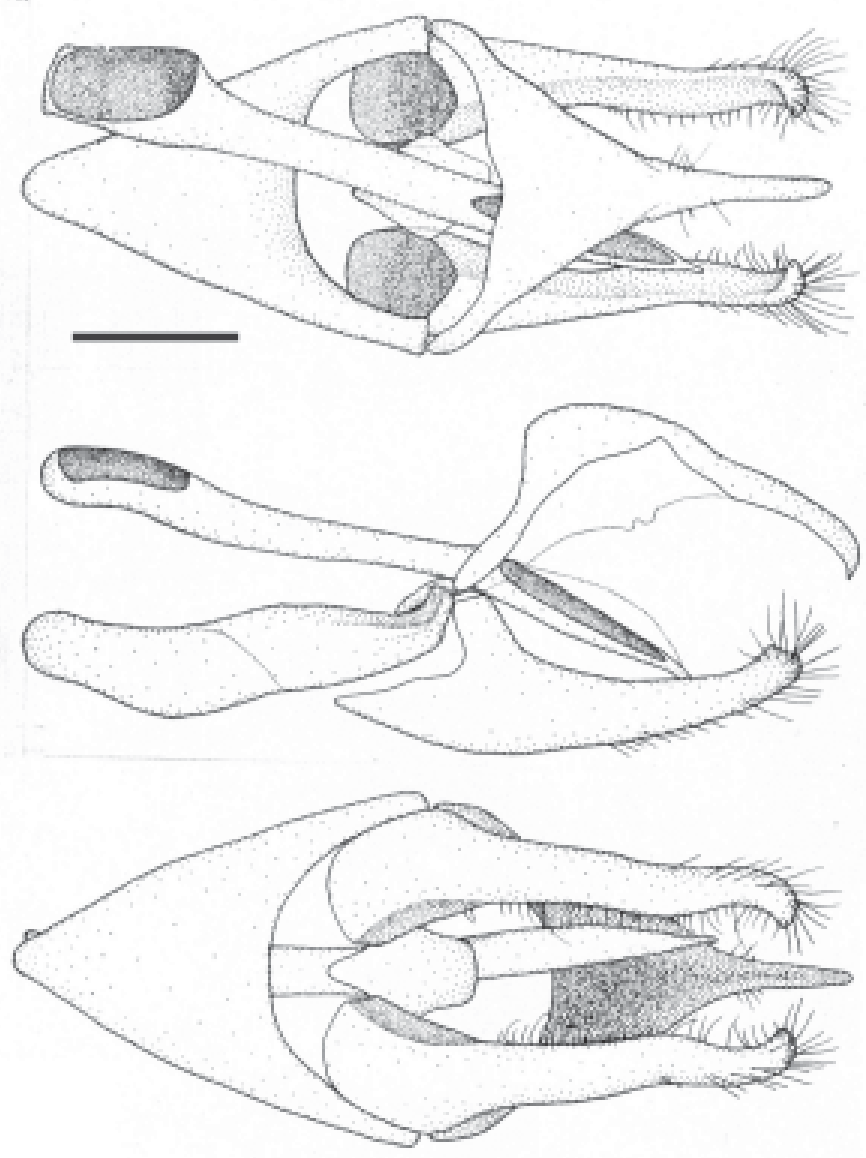

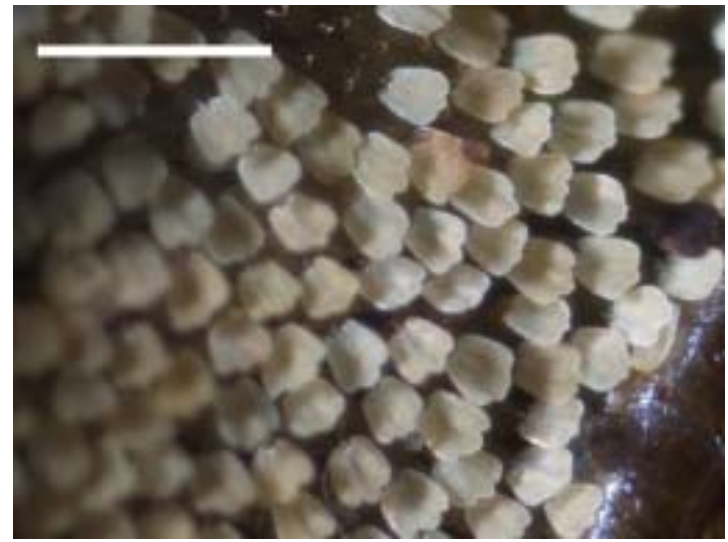

Scales of the basal area of the hindwing Scale bar $0.1 \mathrm{~mm}$

Genitalia in dorsal view Scale bar $1 \mathrm{~mm}$

Genitalia in lateral view Genitalia in ventral view 


\section{Plate 6}

\section{Actinote discrepans}

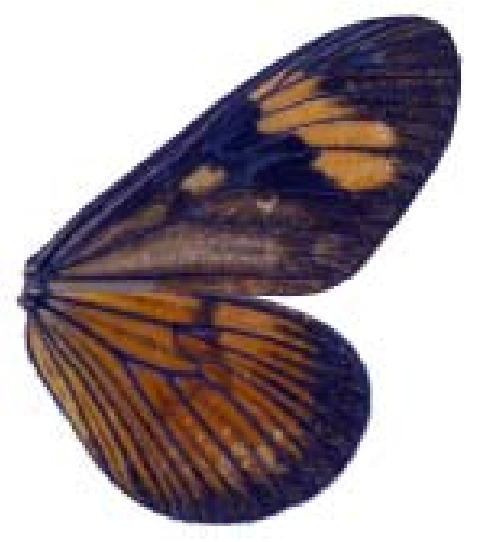

Wings in dorsal view
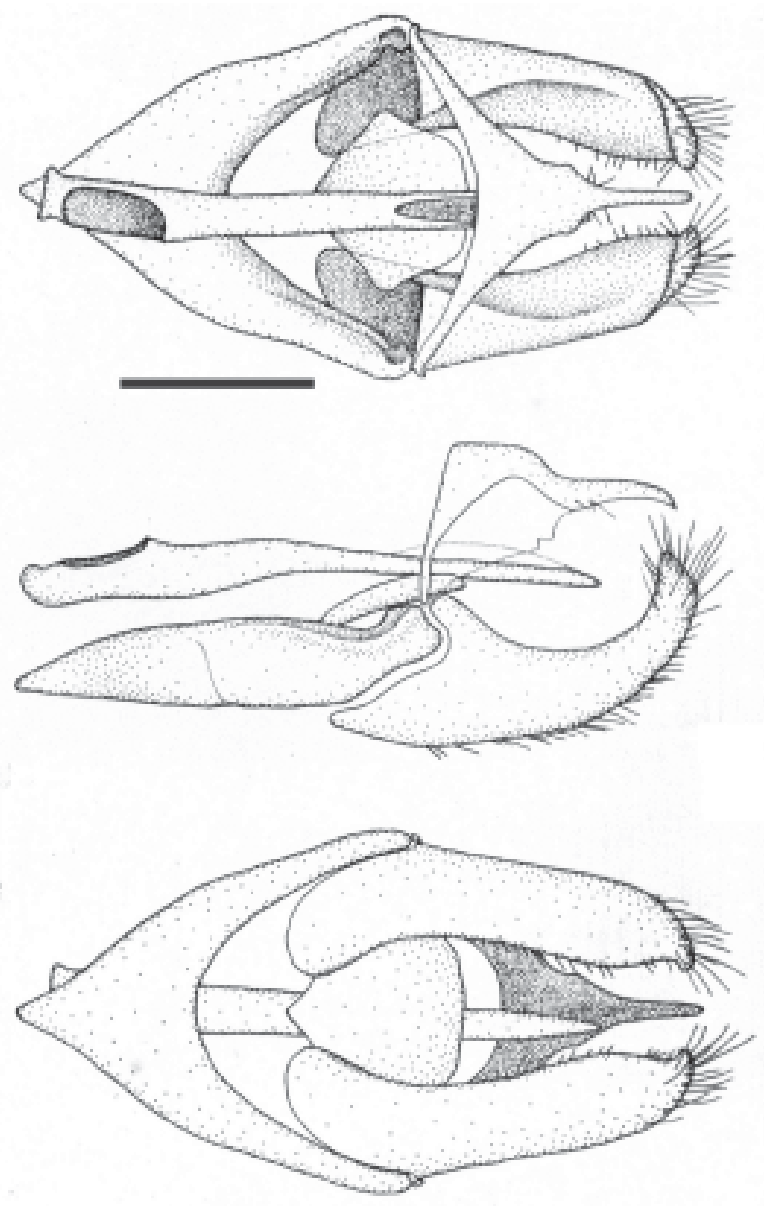

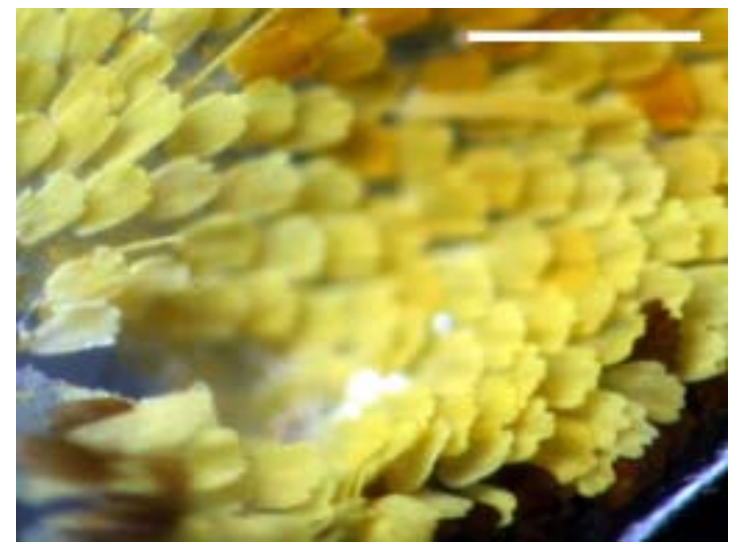

Scales of the basal area of the hindwing Scale bar $0.1 \mathrm{~mm}$

Genitalia in dorsal view Scale bar $1 \mathrm{~mm}$

Genitalia in lateral view

Genitalia in ventral view

http://www.biotaneotropica.org.br 


\section{Plate 7}

\section{Actinote eberti}

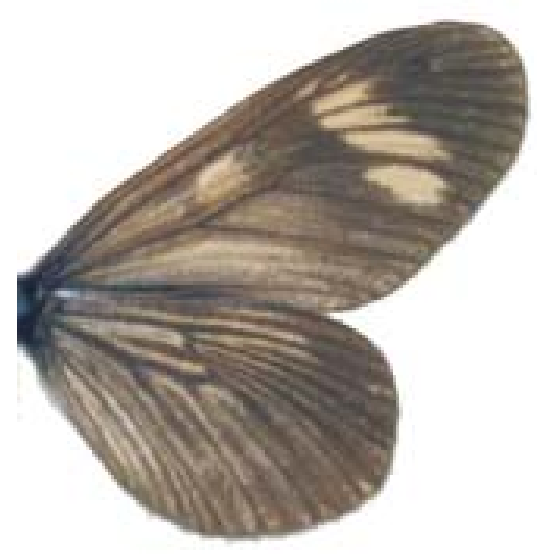

Wings in dorsal view

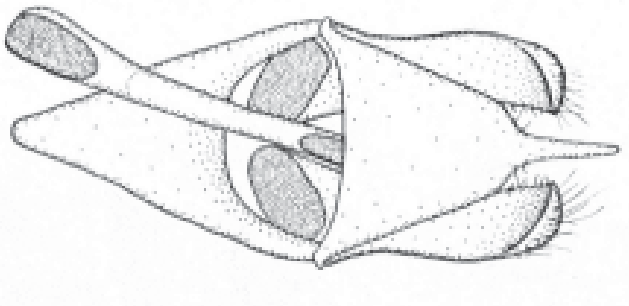

Genitalia in dorsal view Scale bar $1 \mathrm{~mm}$

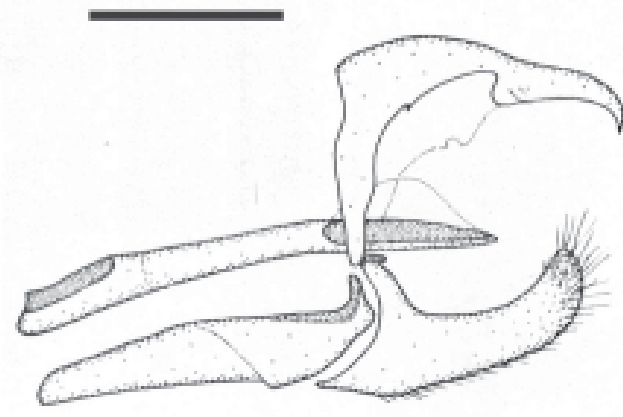

Genitalia in lateral view

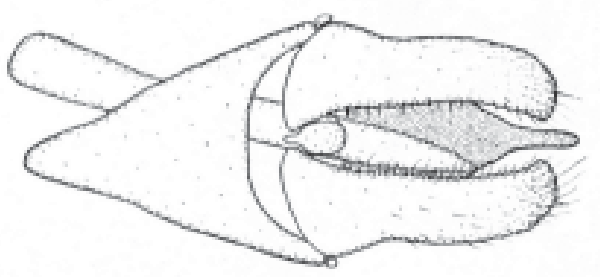

Genitalia in ventral view 


\section{Plate 8}

\section{Actinote melanisans}

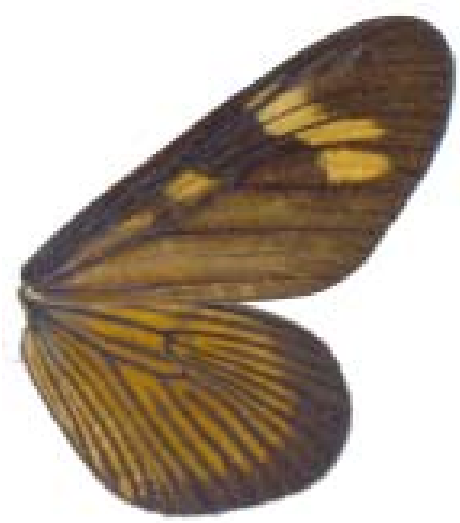

Wings in dorsal view
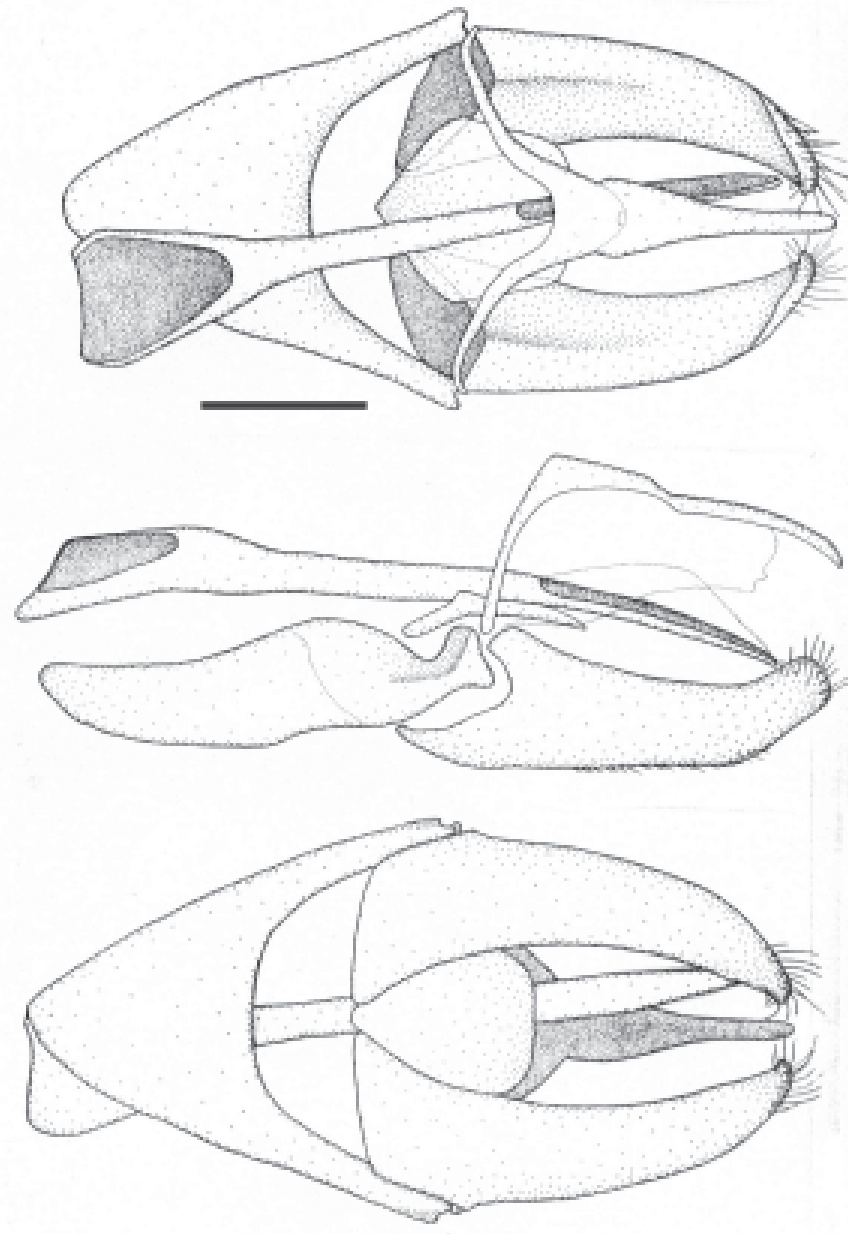

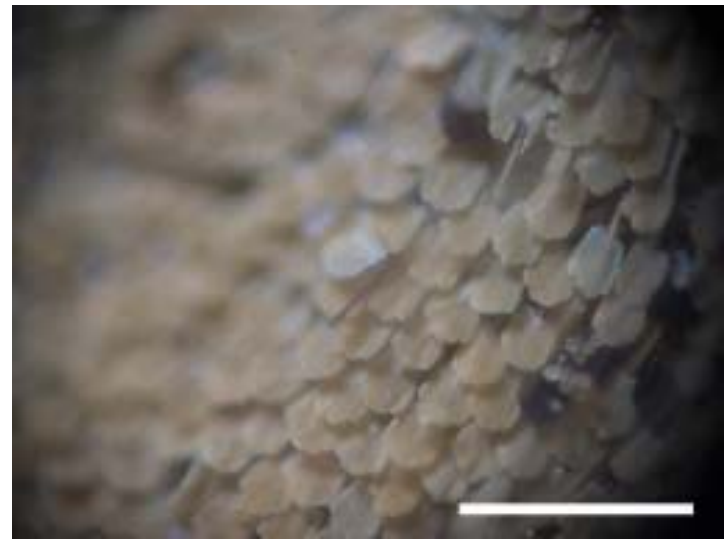

Scales of the basal area of the hindwing Scale bar $0.1 \mathrm{~mm}$

Genitalia in dorsal view Scale bar $1 \mathrm{~mm}$

Genitalia in lateral view

Genitalia in ventral view

http://www.biotaneotropica.org.br 


\section{Plate 9}

\section{Actinote rhodope}

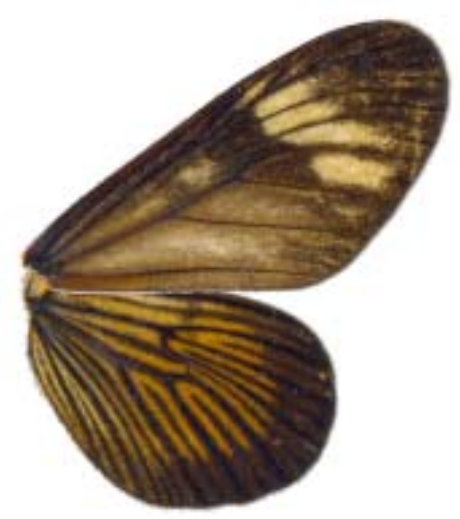

Wings in dorsal view
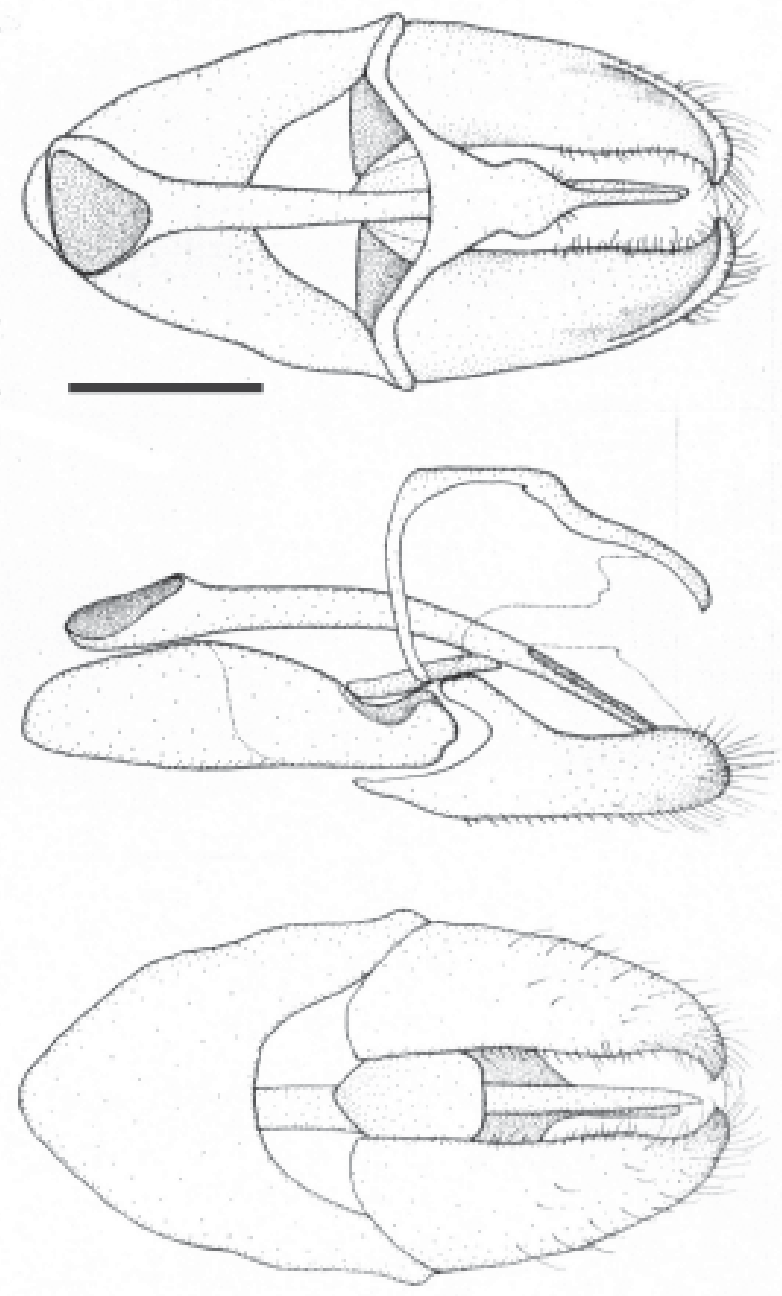

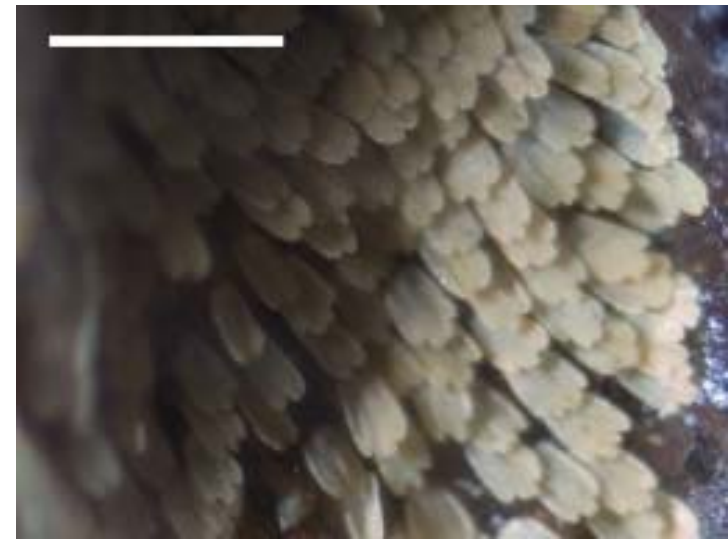

Scales of the basal area of the hindwing Scale bar $0.1 \mathrm{~mm}$

Genitalia in dorsal view Scale bar $1 \mathrm{~mm}$

Genitalia in lateral view

Genitalia in ventral view

http://www.biotaneotropica.org.br 


\section{Plate 10}

\section{Actinote surima}

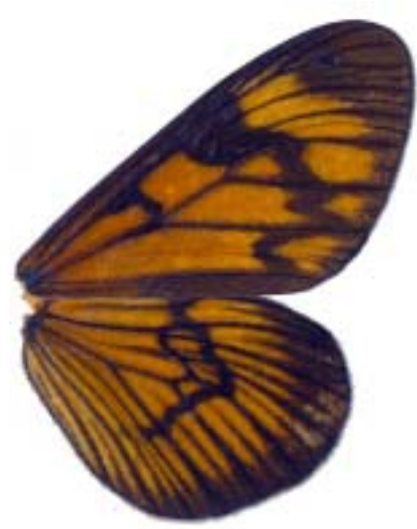

Wings in dorsal view
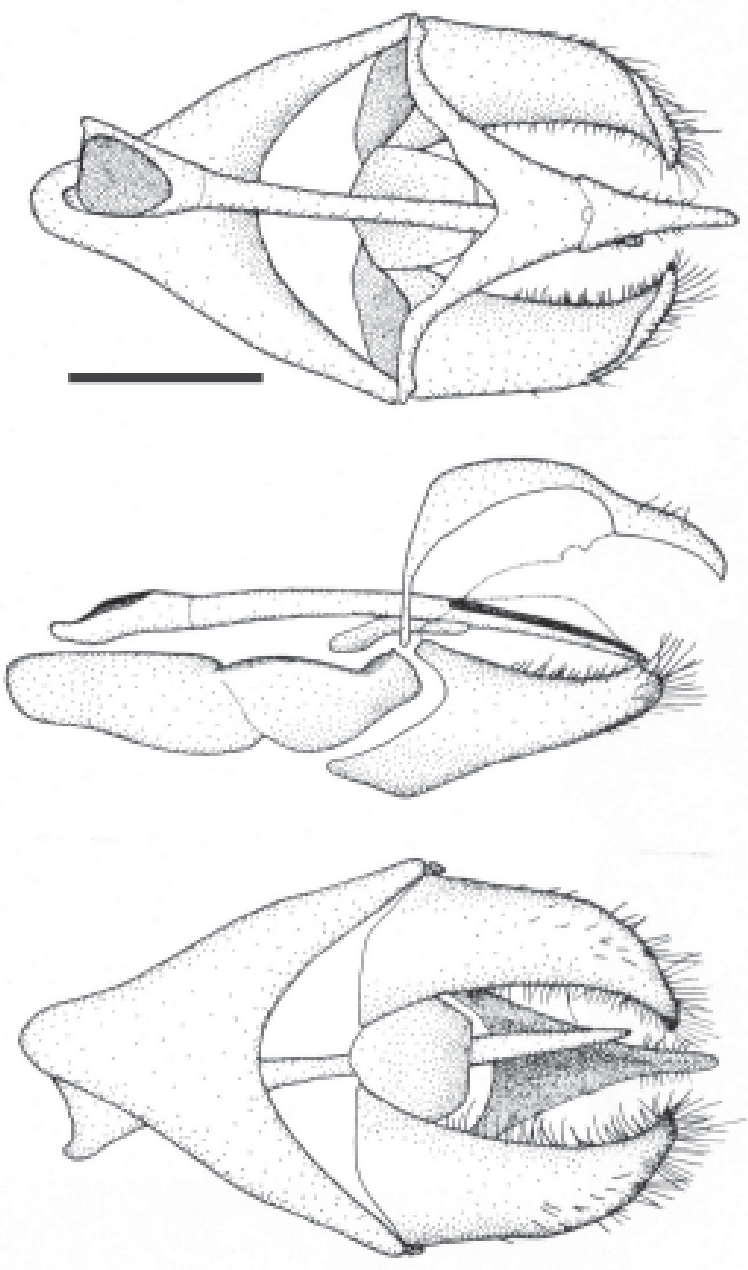

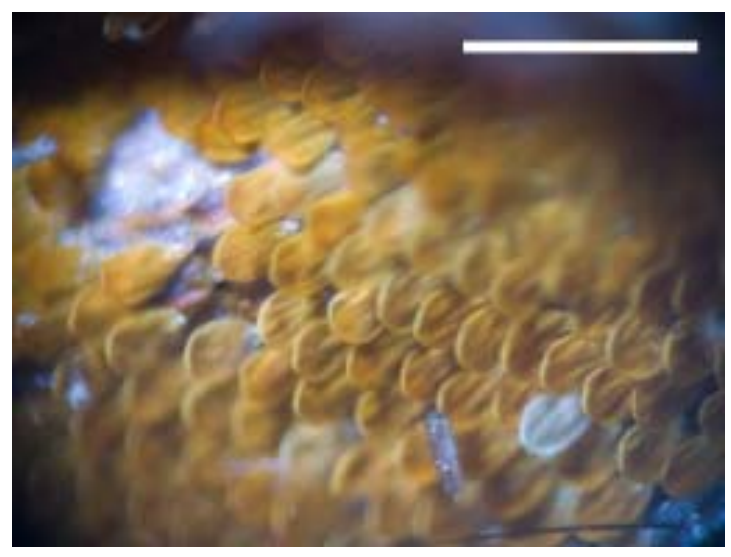

Scales of the basal area of the hindwing Scale bar $0.1 \mathrm{~mm}$

Genitalia in dorsal view Scale bar $1 \mathrm{~mm}$

\section{Genitalia in lateral view}

Genitalia in ventral view

http://www.biotaneotropica.org.br 


\section{Plate 11}

\section{Actinote catarina}

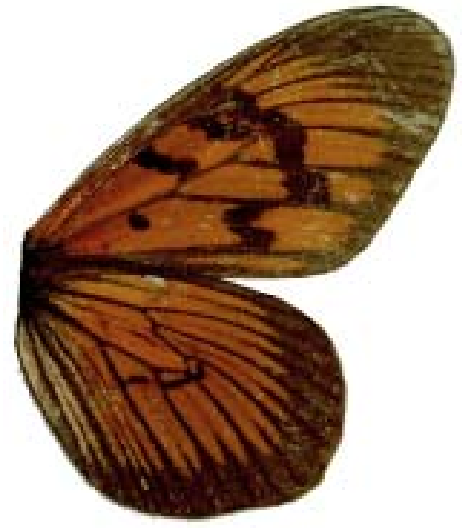

Wings in dorsal view
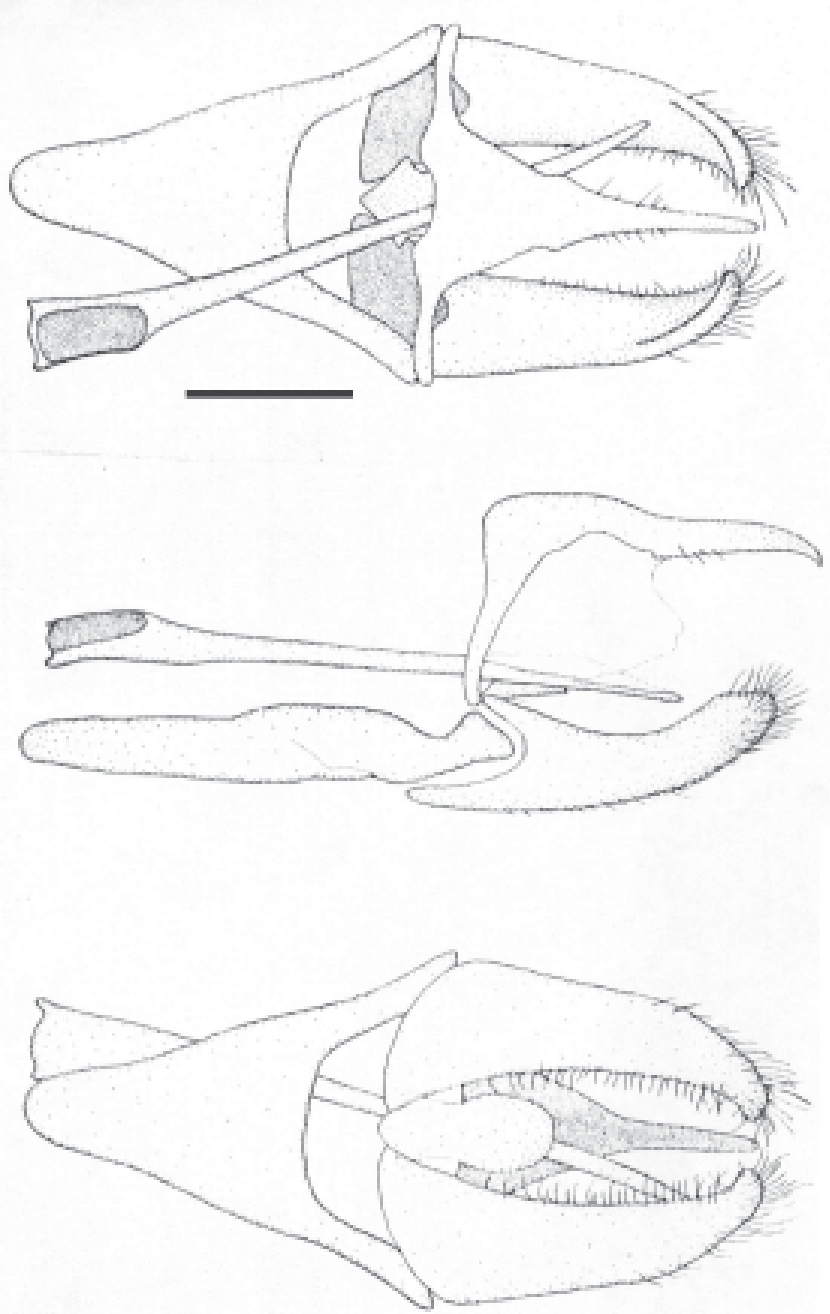

Genitalia in ventral view
Genitalia in dorsal view

Scale bar $1 \mathrm{~mm}$

Genitalia in lateral view

Scales of the basal area of the hindwing Scale bar $0.1 \mathrm{~mm}$

http://www.biotaneotropica.org.br 


\section{Plate 12}

\section{Actinote alalia}

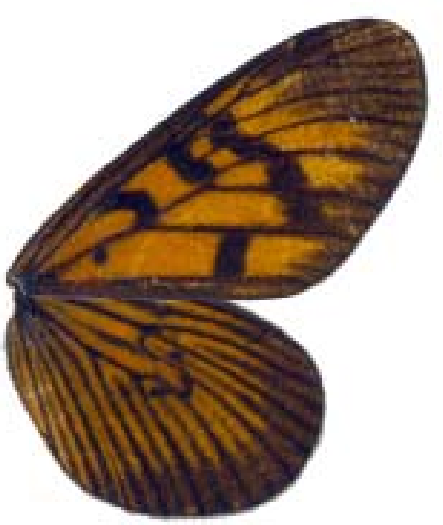

Wings in dorsal view
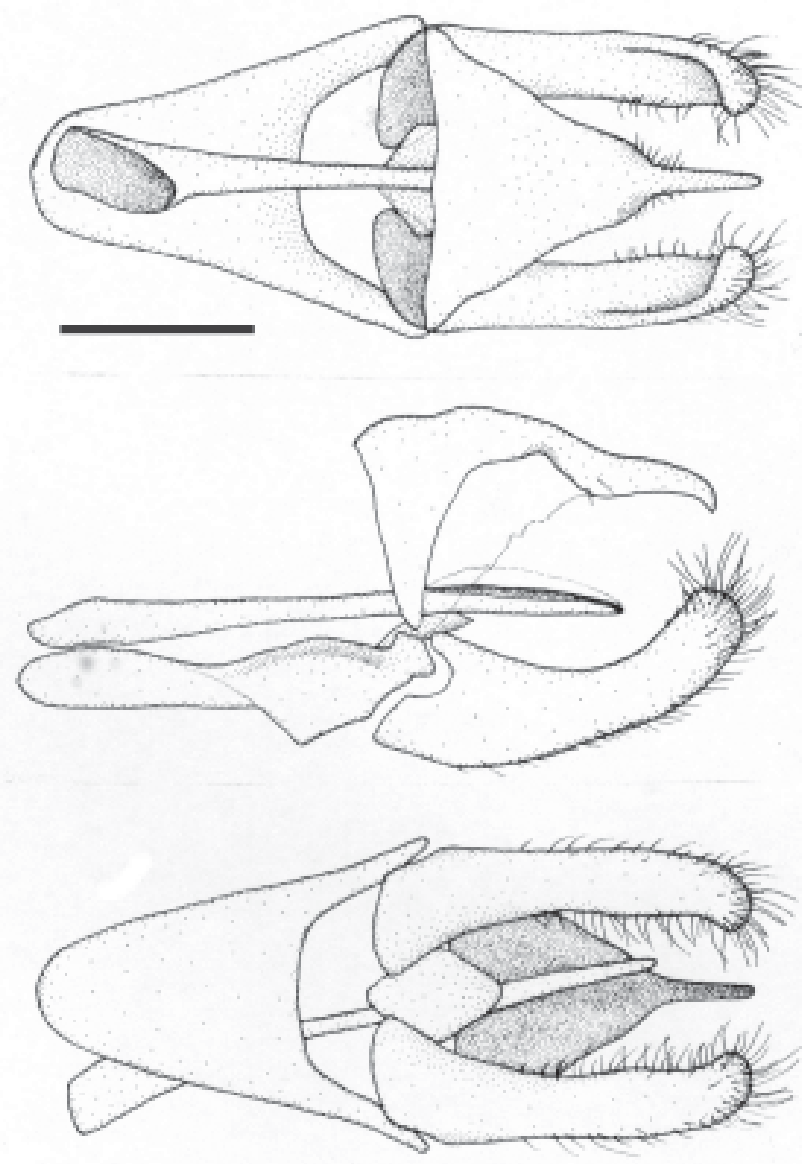

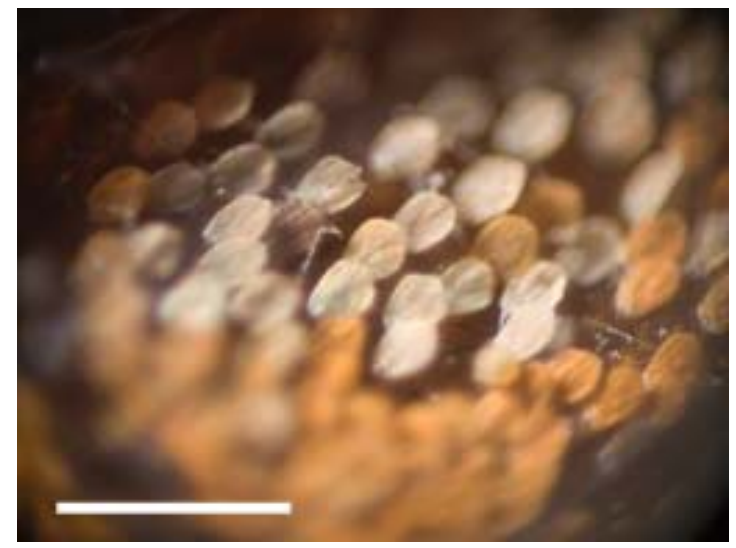

Scales of the basal area of the hindwing Scale bar $0.1 \mathrm{~mm}$

Genitalia in dorsal view Scale bar $1 \mathrm{~mm}$

Genitalia in lateral view

Genitalia in ventral view

http://www.biotaneotropica.org.br 


\section{Plate 13}

\section{Actinote conspicua}

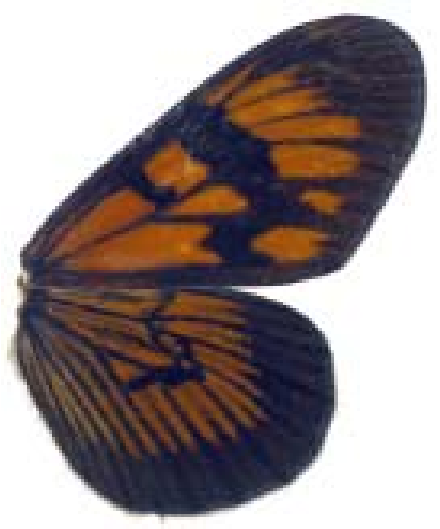

Wings in dorsal view $g$
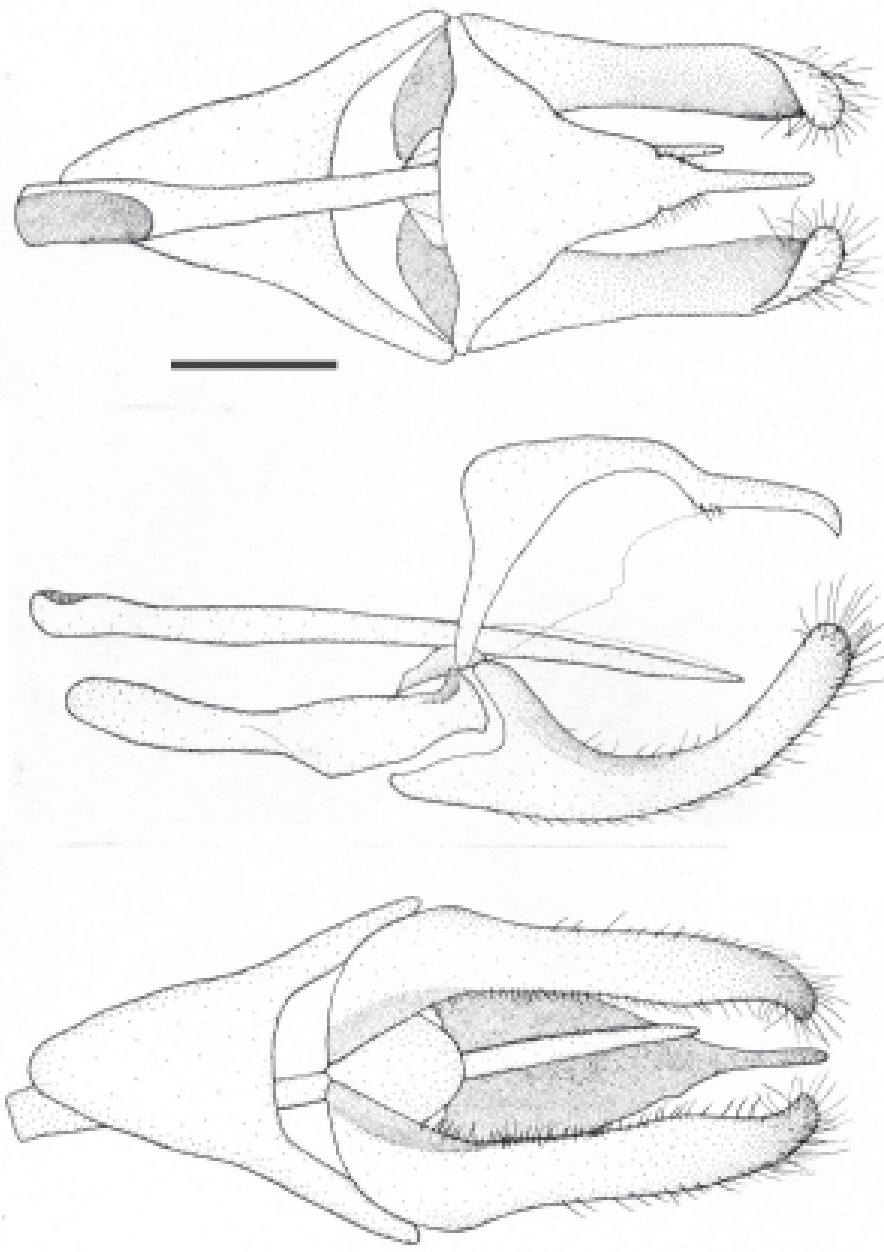

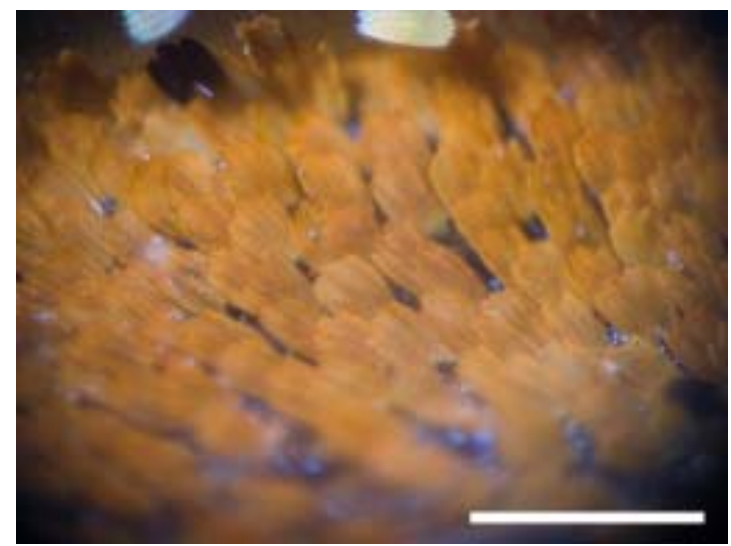

Scales of the basal area of the hindwing Scale bar $0.1 \mathrm{~mm}$

Genitalia in dorsal view Scale bar $1 \mathrm{~mm}$

Genitalia in lateral view

Genitalia in ventral view

http://www.biotaneotropica.org.br 


\section{Plate 14}

\section{Actinote bonita}
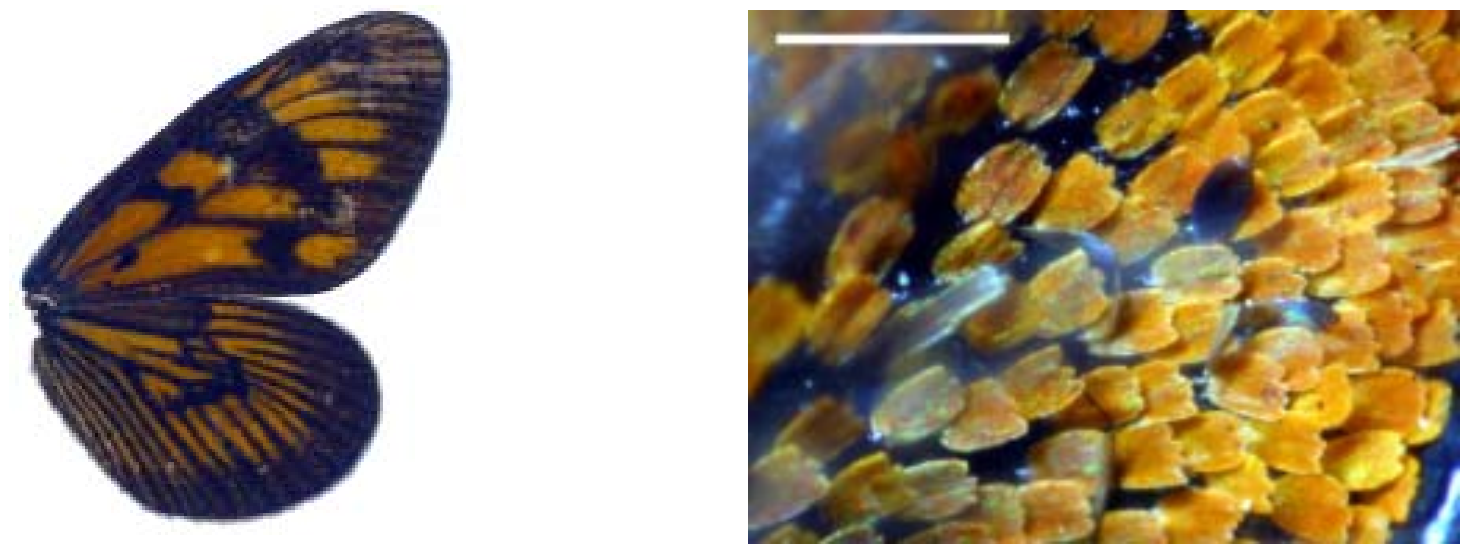

Wings in dorsal view

Scales of the basal area of the hindwing Scale bar $0.1 \mathrm{~mm}$

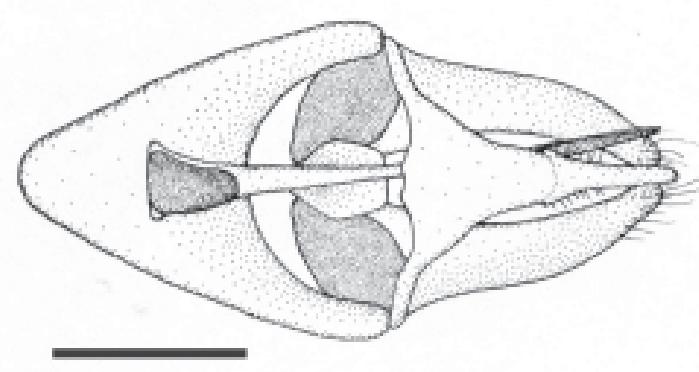

Genitalia in dorsal view

Scale bar $1 \mathrm{~mm}$

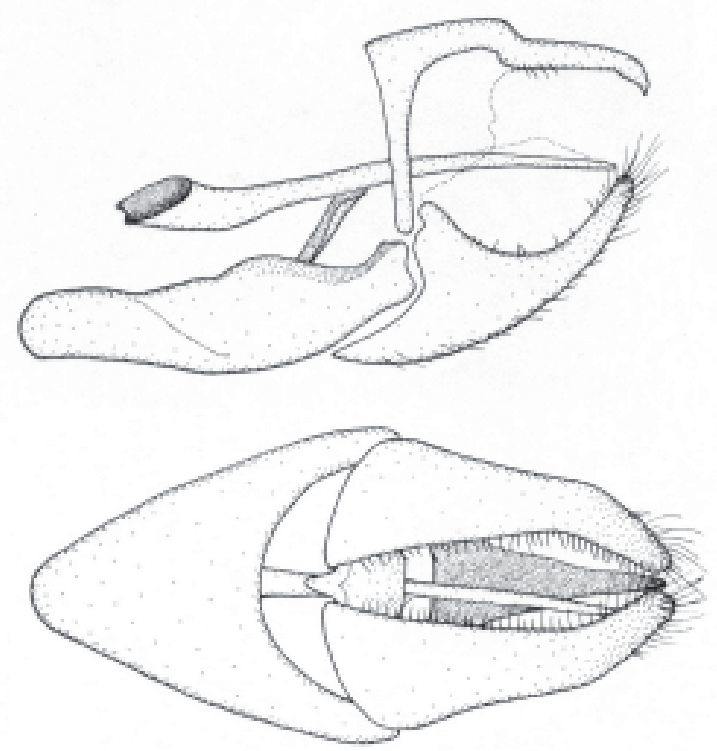

Genitalia in lateral view

Genitalia in ventral view

http://www.biotaneotropica.org.br 


\section{Plate 15}

\section{Actinote dalmeidai}
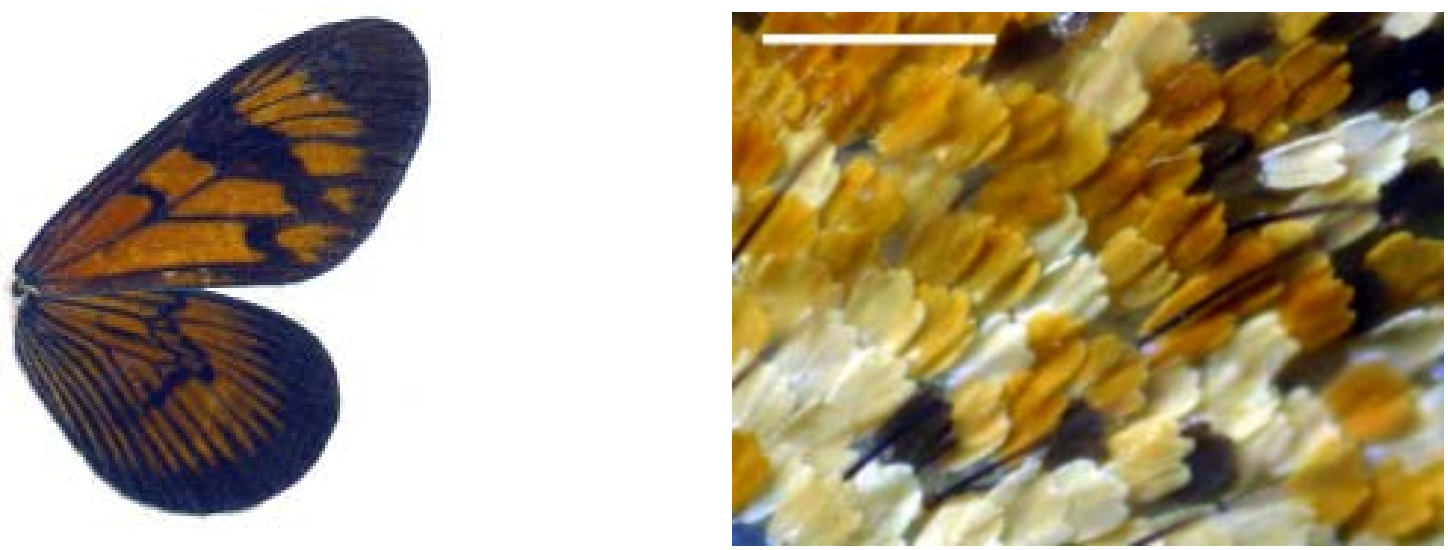

Wings in dorsal view
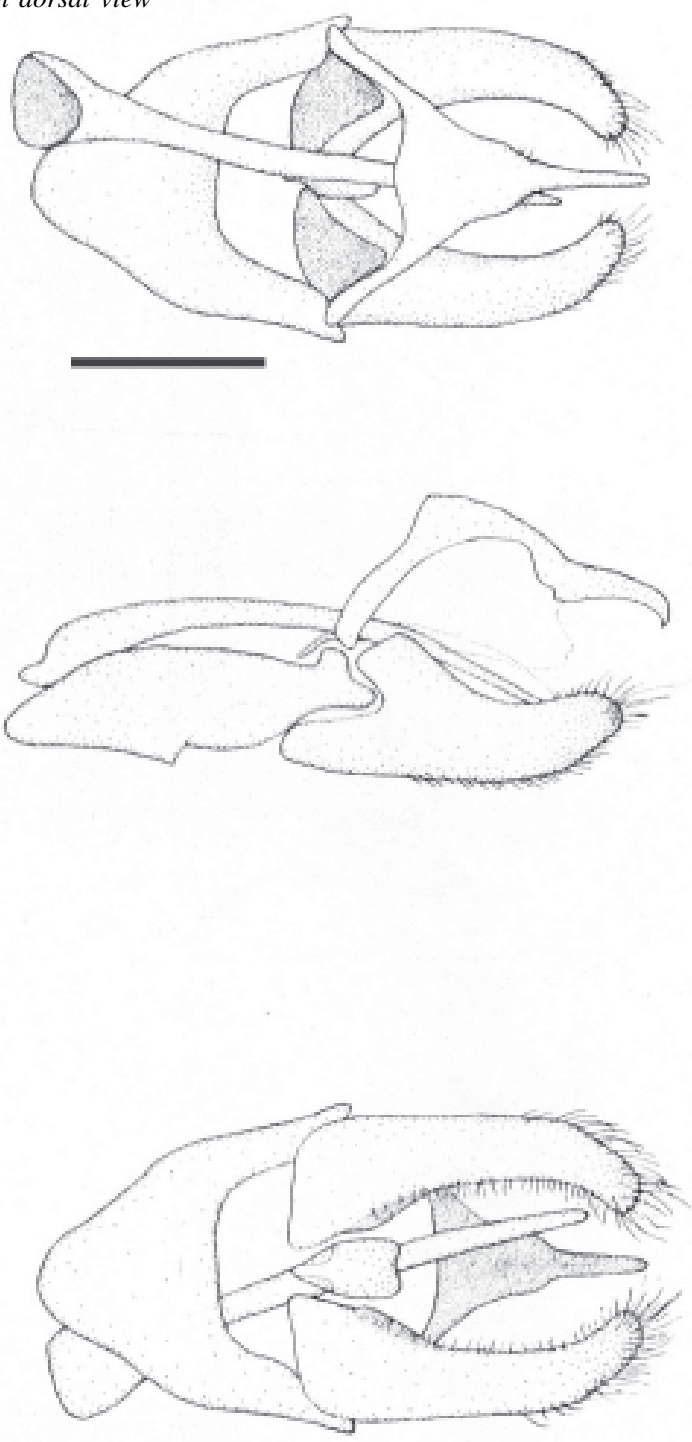

Scales of the basal area of the hindwing Scale bar $0.1 \mathrm{~mm}$

Genitalia in dorsal view Scale bar $1 \mathrm{~mm}$

Genitalia in lateral view

Genitalia in ventral view 


\section{Plate 16}

\section{Actinote quadra}

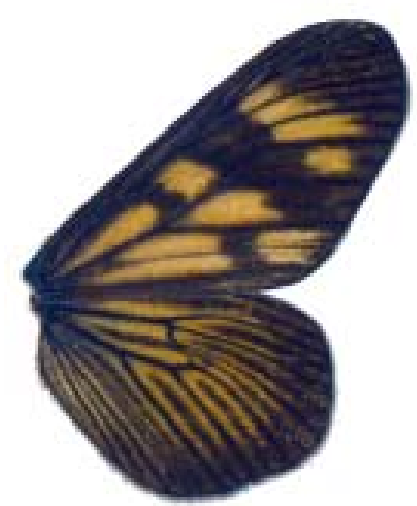

Wings in dorsal view
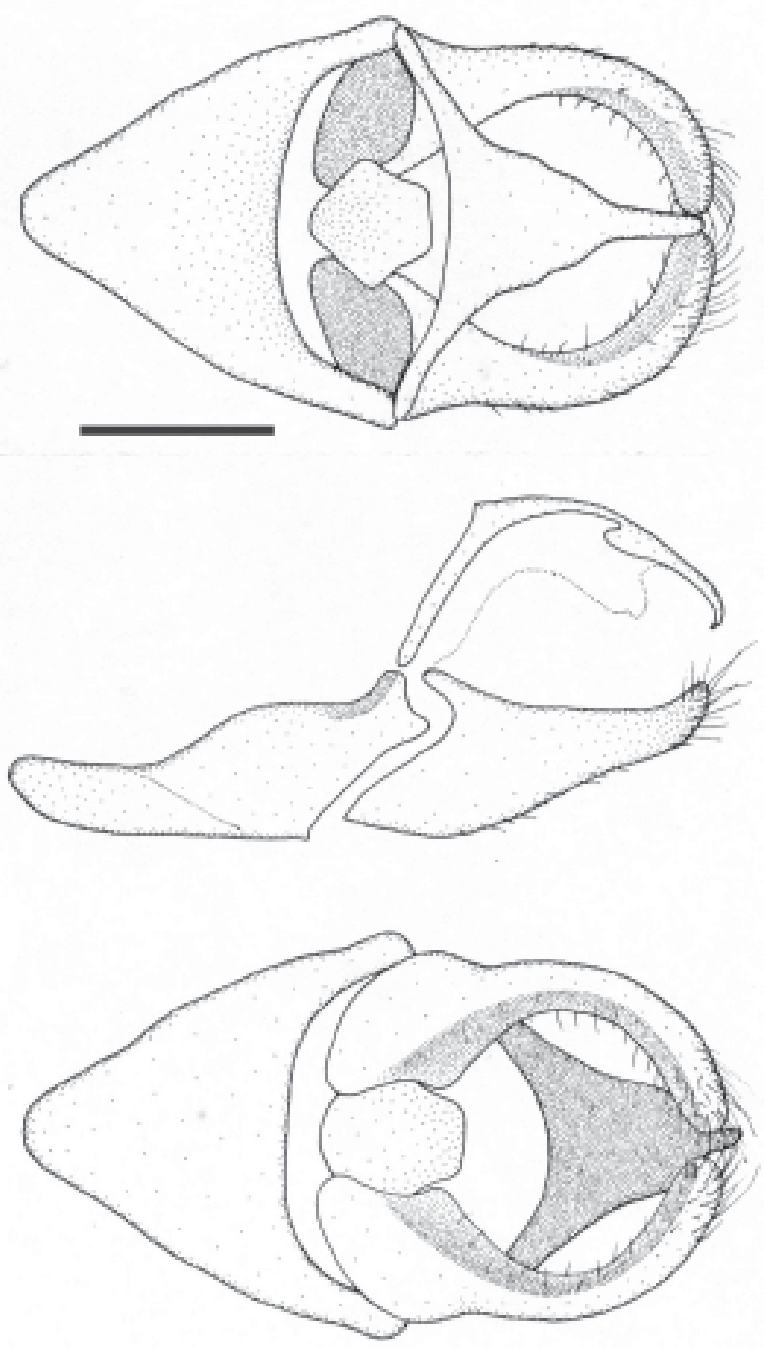

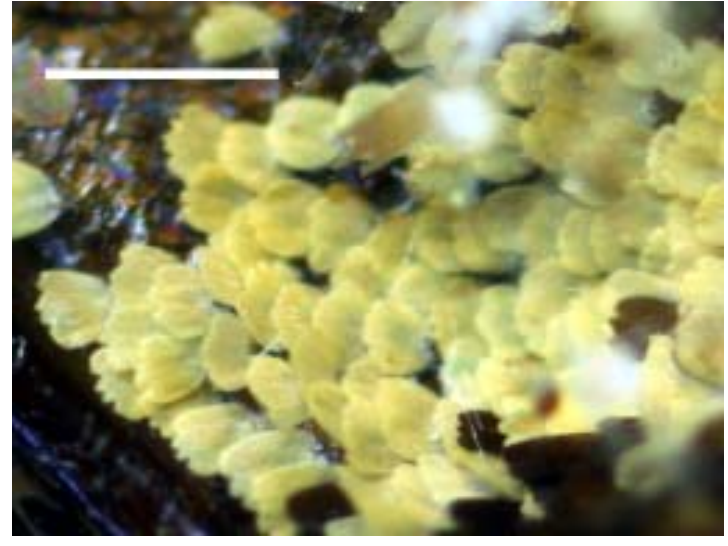

Scales of the basal area of the hindwing Scale bar $0.1 \mathrm{~mm}$

Genitalia in dorsal view Scale bar $1 \mathrm{~mm}$

Genitalia in lateral view

Genitalia in ventral view 


\section{Plate 17}

\section{Actinote parapheles}

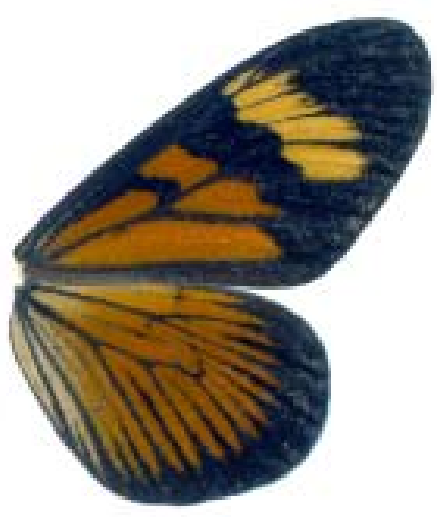

Wings in dorsal view
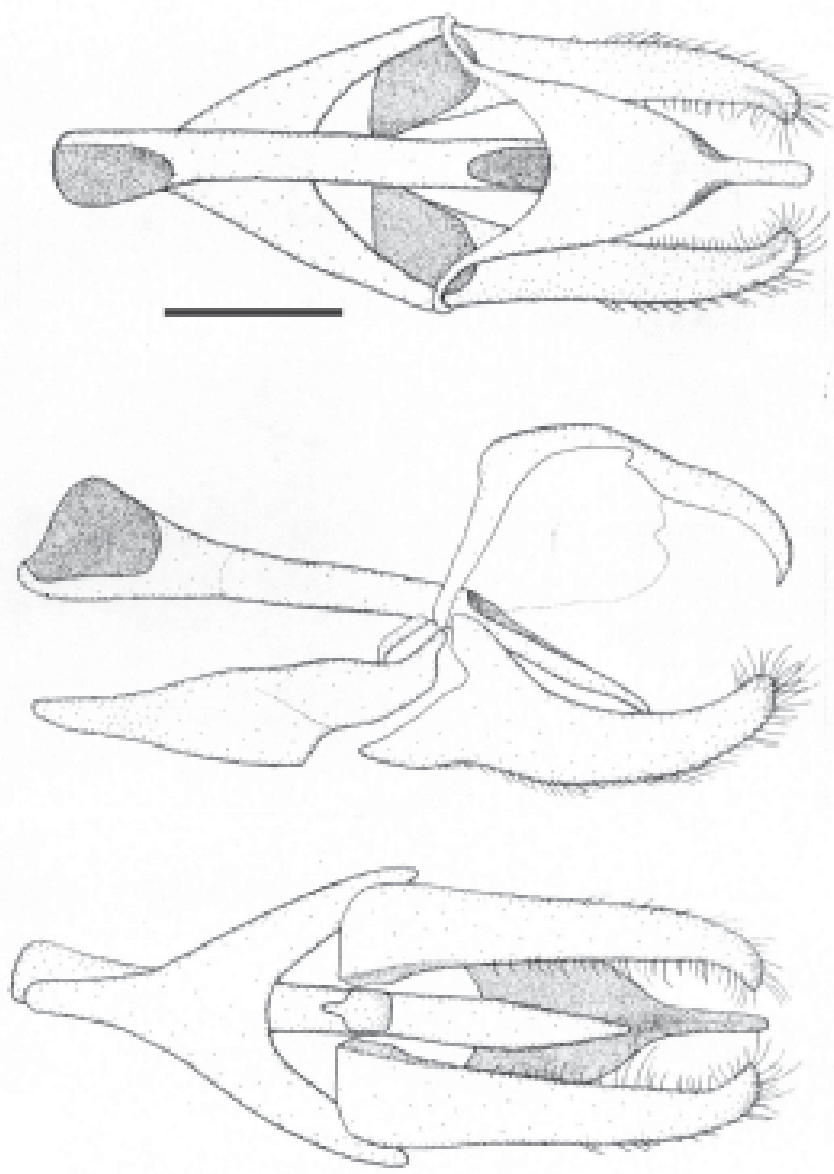

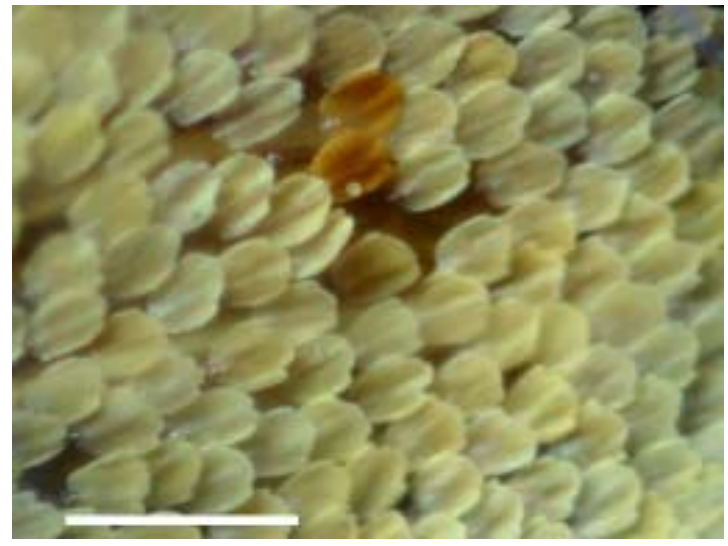

Scales of the basal area of the hindwing Scale bar $0.1 \mathrm{~mm}$

Genitalia in dorsal view Scale bar $1 \mathrm{~mm}$

Genitalia in lateral view Genitalia in ventral view 


\section{Plate 18}

\section{Actinote pellenea}

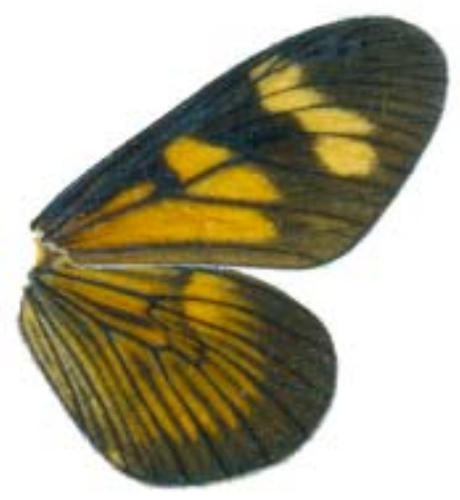

Wings in dorsal view
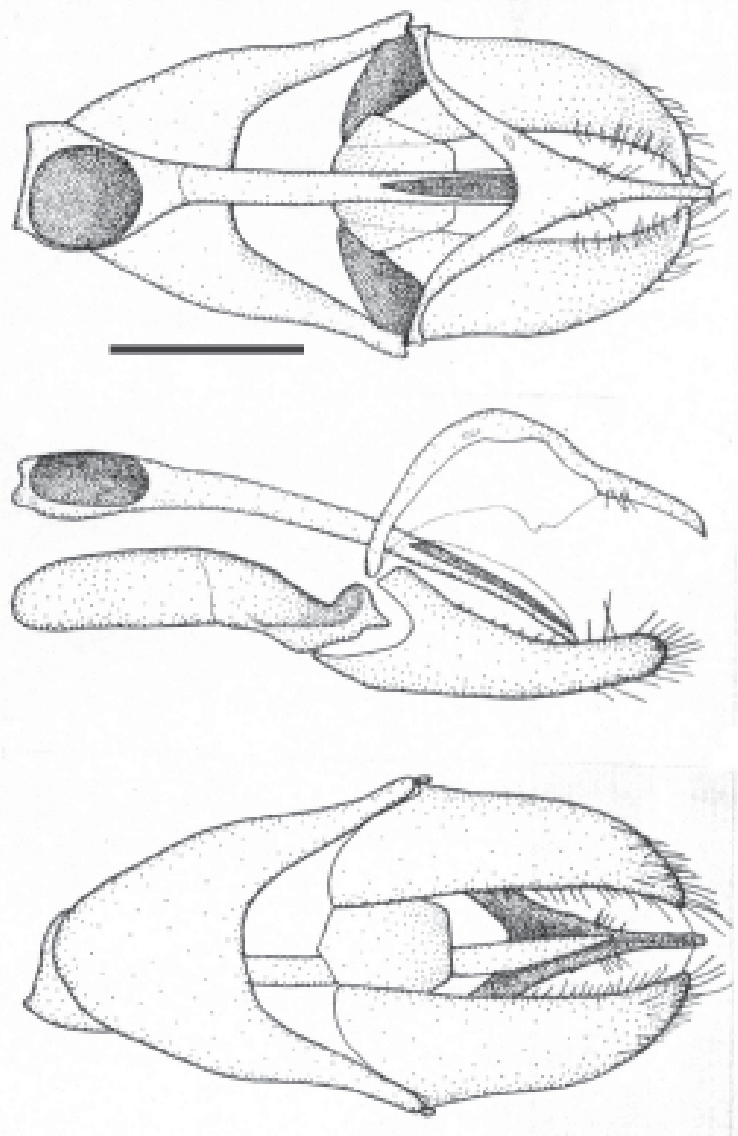

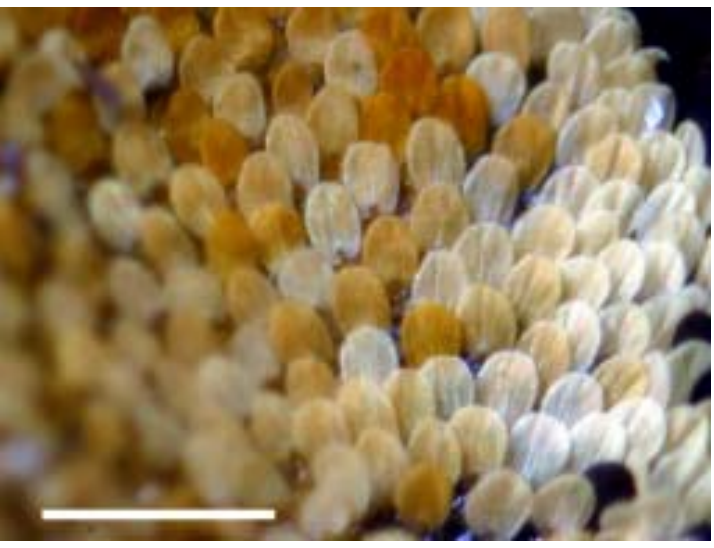

Scales of the basal area of the hindwing Scale bar $0.1 \mathrm{~mm}$

Genitalia in dorsal view

Scale bar $1 \mathrm{~mm}$

Genitalia in lateral view

Genitalia in ventral view

http://www.biotaneotropica.org.br 


\section{Plate 19}

\section{Actinote carycina}
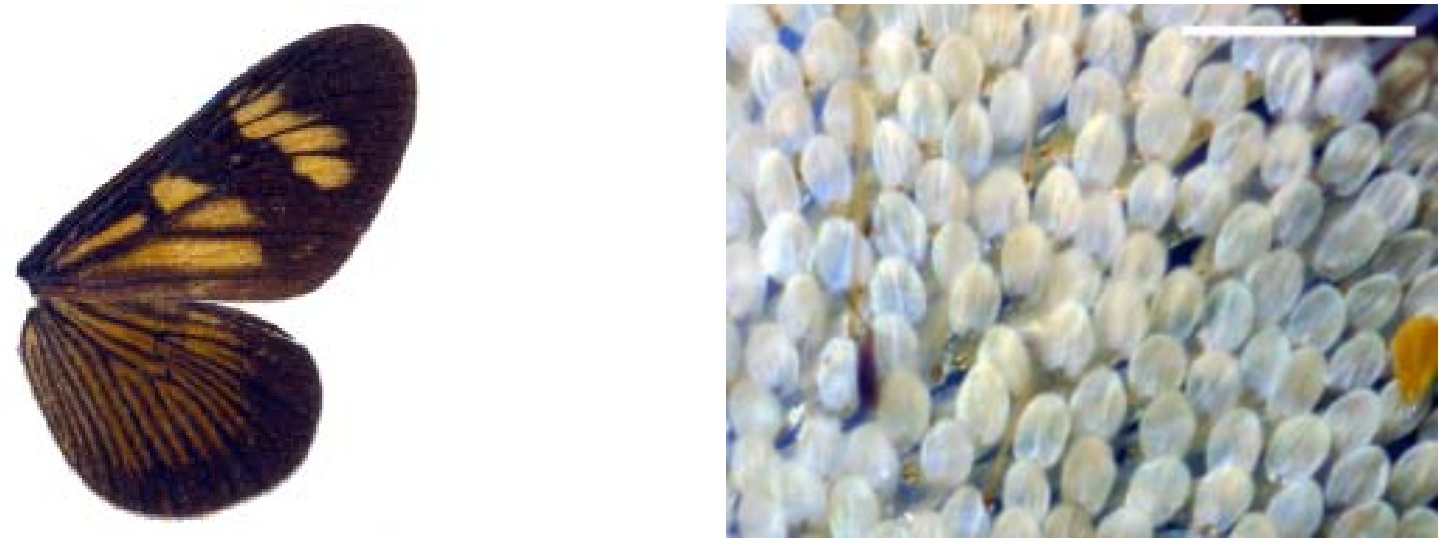

Wings in dorsal view

Scales of the basal area of the hindwing Scale bar $0.1 \mathrm{~mm}$

Genitalia in dorsal view

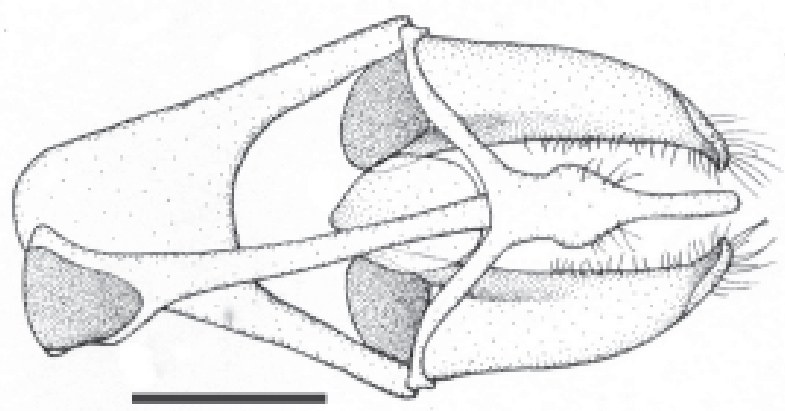

Scale bar $1 \mathrm{~mm}$

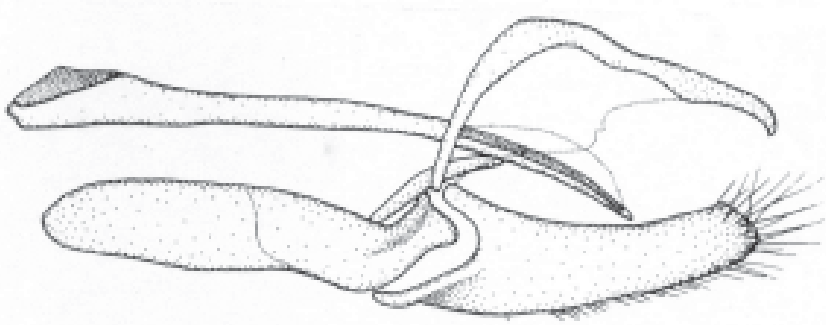

Genitalia in lateral view

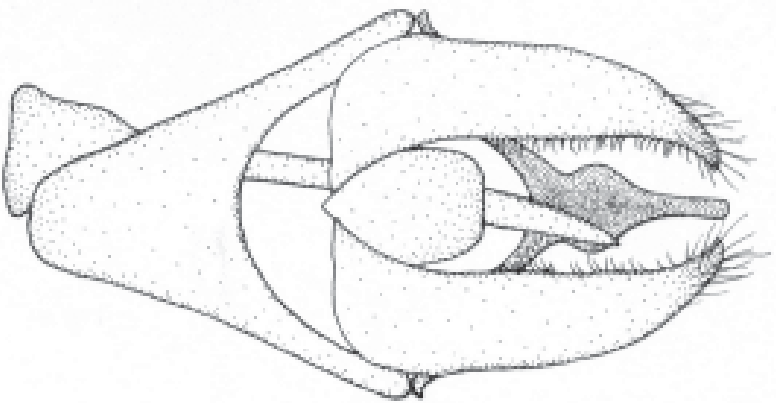

Genitalia in ventral view

http://www.biotaneotropica.org.br 


\section{Plate 20}

\section{Actinote pratensis}
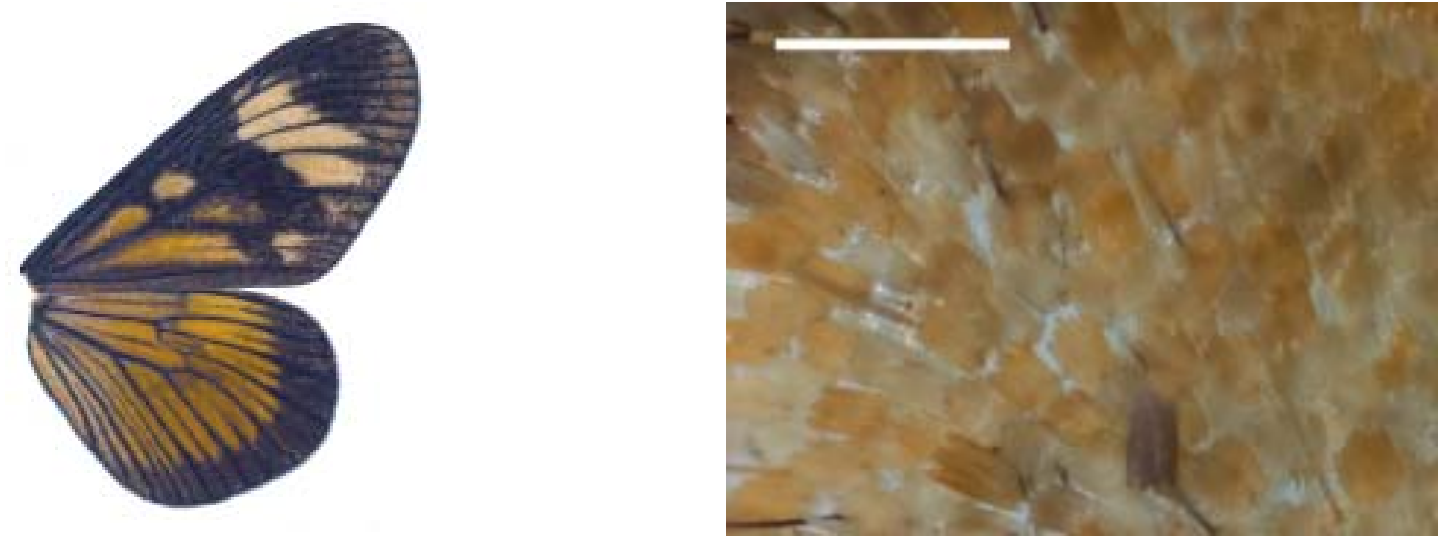

Scales of the basal area of the hindwing Scale bar $0.1 \mathrm{~mm}$

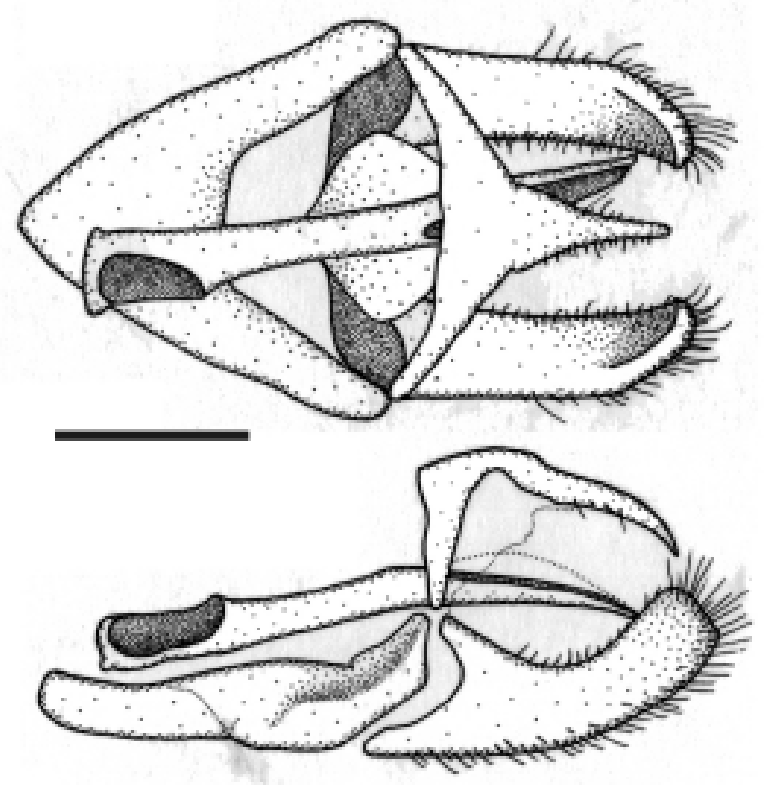

Genitalia in dorsal view Scale bar $1 \mathrm{~mm}$

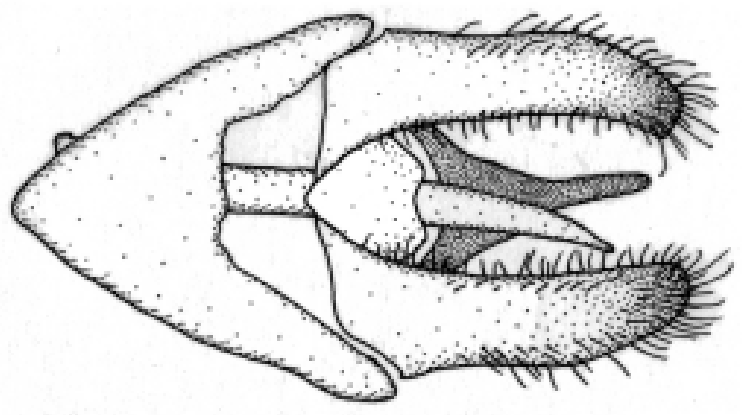

Genitalia in lateral view

Genitalia in ventral view

http://www.biotaneotropica.org.br 


\section{Actinote pyrrha}

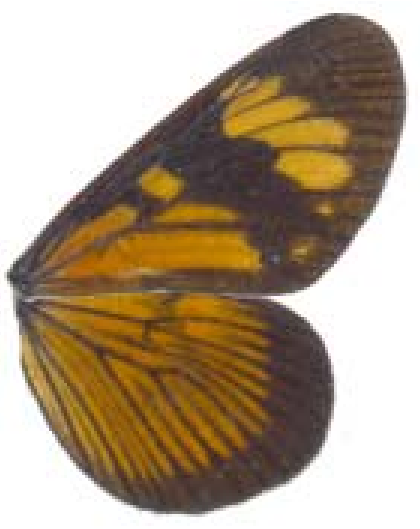

Wings in dorsal view
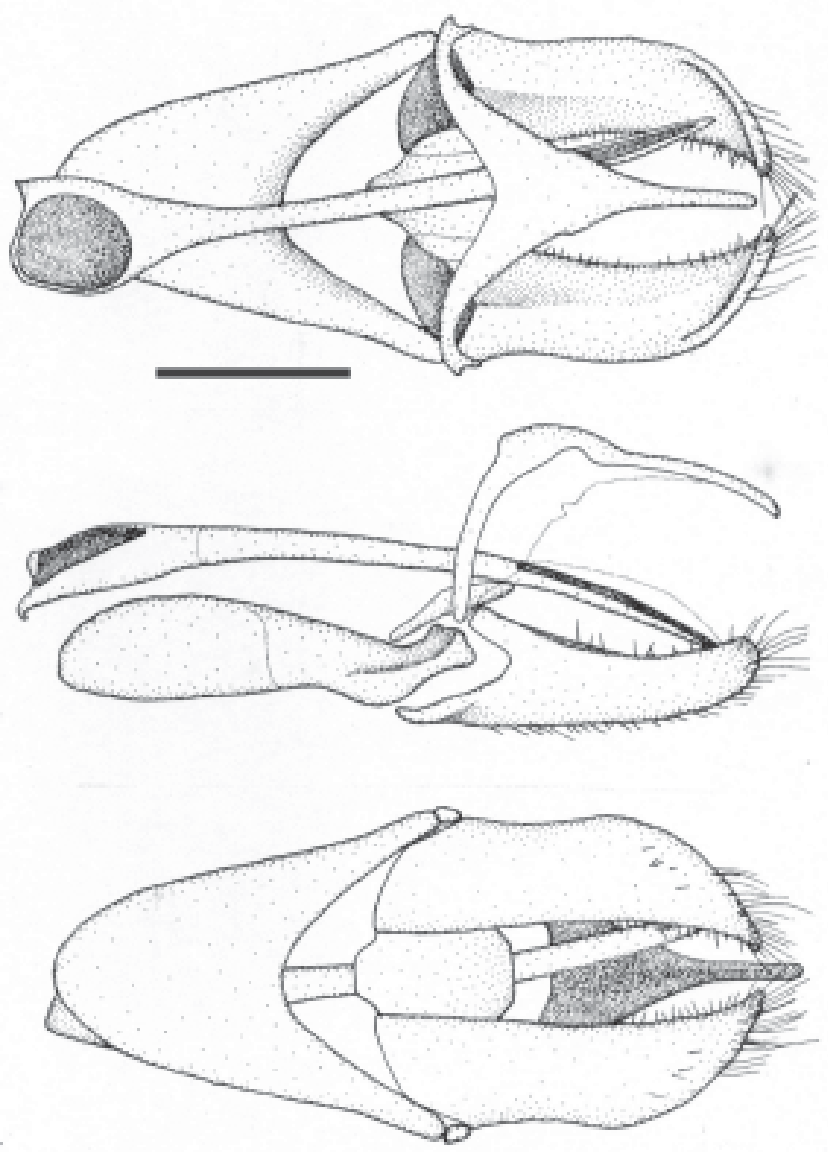

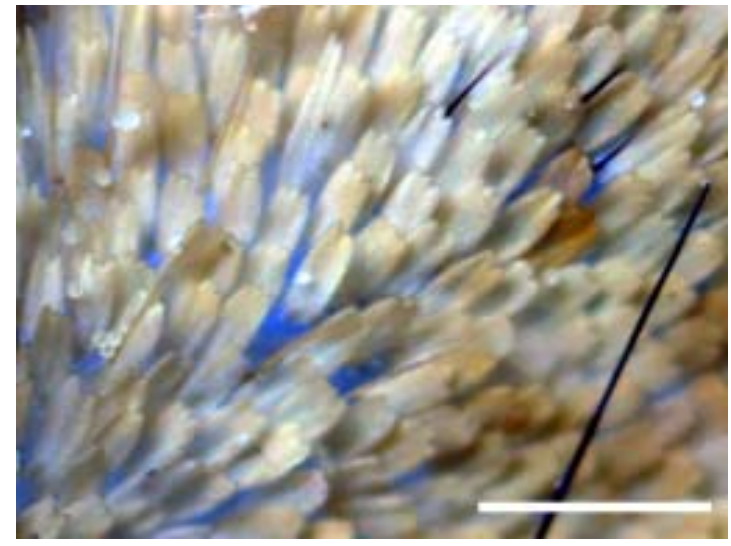

Scales of the basal area of the hindwing Scale bar $0.1 \mathrm{~mm}$

Genitalia in dorsal view Scale bar $1 \mathrm{~mm}$

Genitalia in lateral view

Genitalia in ventral view 


\section{Plate 22}

\section{Actinote brylla}
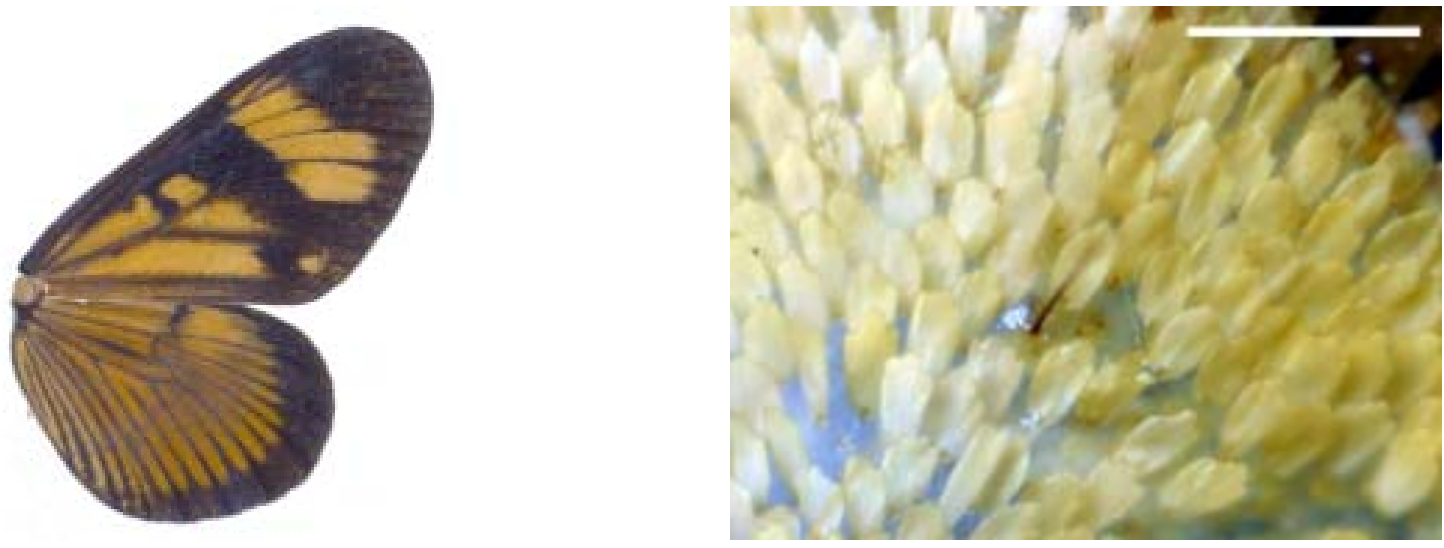

Wings in dorsal view

Scales of the basal area of the hindwing Scale bar $0.1 \mathrm{~mm}$

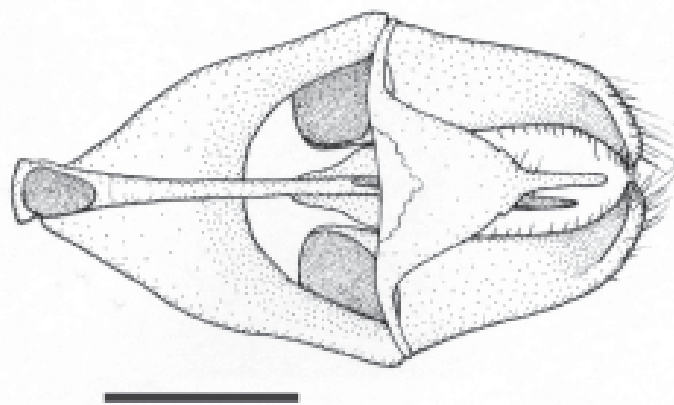

Genitalia in dorsal view

Scale bar $1 \mathrm{~mm}$

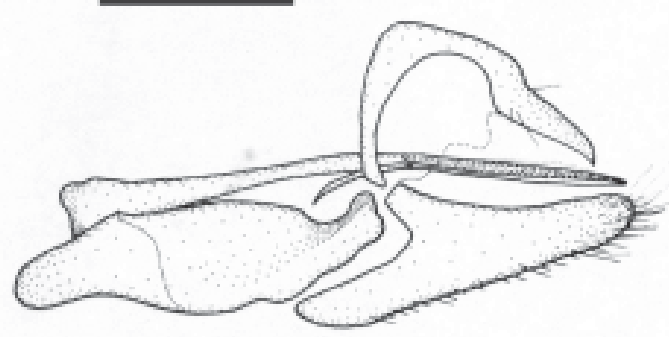

Genitalia in lateral view

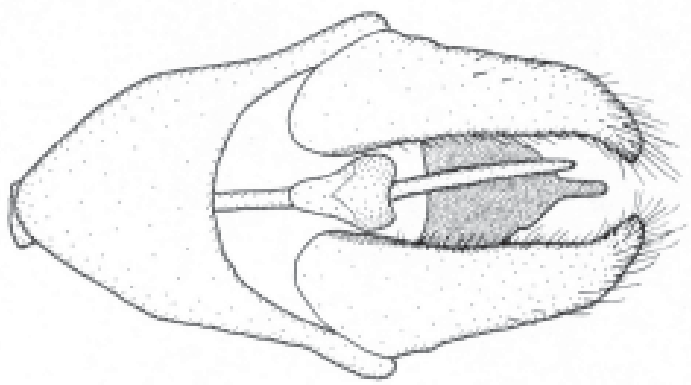

Genitalia in ventral view

http://www.biotaneotropica.org.br 


\section{Acknowledgments}

Numerous colleagues and friends encouraged us to produce this key, and we are grateful to all. For access to collections and help in the initial development of our work, we thank Olaf Mielke and Mirna Casagrande (Universidade Federal do Paraná), Fernando Meyer (Museu Anchieta, Porto Alegre), Phil Ackery (The Natural History Museum, London), and Bob Robbins (Smithsonian Institution). Phil DeVries and Andrew Neild provided discussion and useful comments to early versions of the manuscript. Special thanks to Márlon Paluch and an anonymous reviewer for their detailed suggestions. This work was funded by FAPESP (fellowships to RBF) and the National Science Foundation (DEB 0527441, to CMP).

\section{References}

Ackery, P.R. 1984. Systematic and faunistic studies on butterflies. Symposium of the Royal Entomological Society, London, 11:9-21.

Ackery, P.R. 1988. Hostplants and classification: a review of nymphalid butterflies. Biological Journal of the Linnean Society, London, 33:95-203.

Almeida, G.S.S., C.L. Souza \& E.E. Marques. 1986. Levantamento preliminar das espécies de borboletas (Rhopalocera) de ocorrência em Maringá (PR). I. Papilionoidea. Revista UNIMAR, Maringá, 8(1):29-36.

Araújo, A.M. \& V.L.S. Valente. 1981. Observações sobre alguns lepidópteros e drosofilídeos do Parque do Turvo, RS. Ciência e Cultura, 33(11):1485-1490.

Aurivillius, P.O.C. 1882. Recensio critica Lepidopterorum Musei Ludovicae Ulricae quae descripsit Carolus a Linné. Kongliga Svenska Vetenskaps-Akademiens Handlingar (Ny Följd) 19(5):1-188, 1 plate, 4 figs.

Bates, H.W. 1862. Contributions to an insect fauna of the Amazon valley. Lepidoptera: Heliconiidae. Transactions of the Linnean Society, 23:495-566.

Biezanko, C.M. 1938. Dois meses de caça lepidopterológica em arredores de Porto União e União da Vitória. O Campo, Rio de Janeiro, 10(109):38-40.

Biezanko, C.M. \& R.G. Freitas. 1938. Catálogo dos insetos encontrados na cidade de Pelotas e seus arredores. I. Lepidoptera. Contribuição ao conhecimento da fisiografia do Rio Grande do Sul. Boletim da Escola de Agronomia Eliseu Maciel, Pelotas, 25:1-30.

Biezanko, C.M. \& J. Pitoñ. 1941. Breves apontamentos sobre alguns lepidópteros encontrados nos arredores de Itaiópolis. Boletim da Escola de Agronomia Eliseu Maciel, Pelotas, 28:1-21.

Biezanko, C.M. \& A. Ruffinelli. 1957. Los lepidópteros de la colección Schweizer. Revista de la Sociedad Uruguaya de Entomologia, Montevideo, 2(1):31-53.
Biezanko, C.M., A. Ruffinelli \& C.S. Carbonell. 1957. Lepidoptera del Uruguay. Lista anotada de espécies. Revista de la Facultad de Agronomia, Montevideo, 46:1-152.

Biezanko, C.M. \& F.D. Seta. 1939. Catálogo dos insetos encontrados em Rio Grande e seus arredores. I. Lepidópteros. A “Universal” Echenique \& Cia., Pelotas.

Bönninghausen, V. 1896. Beitrag zur Kenntnis der Lepidopteren-Fauna von Rio de Janeiro. Verhandlungen des Vereins für Naturwissenschaftliche Unterhaltung zu Hamburg, Hamburg, 9:19-41.

Brown Jr., K.S. 1987. São Paulo (Season Summary). News of the Lepidopterist's Society, 12:41.

Brown Jr., K.S. 1988. Brazil (Season Summary). News of the Lepidopterist's Society, 2:36.

Brown Jr., K.S. 1991. Conservation of Neotropical environments: Insects as indicators. In: N.M. Collins \& J.A. Thomas (ed.), The conservation of insects and their habitats, pp.349-404. Academic Press, NY.

Brown Jr., K.S. 1992. Borboletas da Serra do Japi: diversidade, hábitats, recursos alimentares e variação temporal. In: L.P.C.Morellato (org.), História natural da Serra do Japi. Ecologia e preservação de uma área florestal no sudeste do Brasil. pp. 142-186. Editora da UNICAMP/ FAPESP, Campinas.

Brown Jr., K.S. \& W.W. Benson. 1974. Adaptive polimorphism associated with multiple müllerian mimicry in Heliconius numata (Lep. Nymph.). Biotropica, 6(4):205-228.

Brown Jr., K.S. \& R.B. Francini. 1990. Evolutionary strategies of chemical defense in aposematic butterflies: cyanogenesis in Asteraceae-feeding American Acraeinae. Chemoecology, 1(2):52-56.

Brown Jr., K.S. \& O.H.H. Mielke. 1967. Lepidoptera of the Central Brazil plateau. I. Preliminary list of Rhopalocera: introduction, Nymphalidae, Libytheidae. Journal of the Lepidopterist's Society, 21(2):77-106.

Brown Jr., K.S. \& O.H.H. Mielke. 1968. Lepidoptera of the Central Brazil plateau. III. Partial list for the Belo Horizonte area showing the character of the southeastern "blend zone". Journal of the Lepidopterist's Society, 22(3):147-157.

Brown Jr., K.S. \& O.H.H. Mielke. 1972. The Heliconians of Brazil (Lepidoptera: Nymphalidae). Part II. Introduction and general comments, with a supplementary revision of the tribe. Zoologica (New York) 57(1):1-40.

Burmeister, H.C.C. 1861. Reise durch die La Plata-Staaten, mit besonderer Rücksicht auf die physiche Beschaffenheit und den Culturzustand der Argentinischen Republik. Ausgeführt in den Jahren 1857, 1858, 1859 und 1860. H.W.Schmidt, Halle, 1:VIII + 504 pp; 2:V+ 540 pp. 
Burmeister, H.C.C. 1878. Description physique de la République Argentine, d'après des observations personelles et étrangeres. Lepidopteres. Paul Emile Coni, Paris et Buenos Aires, 5:VI + 524 pp.

Burmeister, H.C.C. 1879. Briefliche Mittheilungen. Stettiner Entomologische Zeitung 40(4/6):194-209.

Butler, A.G. 1870. Catalogue of diurnal Lepidoptera described by Fabricius in the collection of the British Museum. British Museum, London, V+303 pp. 3 plates.

Capronnier, J.B. 1874. Notice sur les époques d'apparition des lépidoptères diurnes du Brésil recueillis par M. C. van Volxen, dans son voyage en 1872. Annales de la Societe Entomologique de Belgique, 17:5-39, 1 plate.

Cardoso, A. 1949. Lepidópteros de Alagoas. Revista de Entomologia, Rio de Janeiro, 20(1-3):427-436.

Chai, P. 1986. Field observations and feeding experiments on the responses of rufous-tailed jacamars (Galbula ruficauda) to free-flying butterflies in a tropical rainforest. Biological Journal of the Linnean Society, 29:161-189.

Chai, P. 1996. Butterfly visual characteristics and ontogeny of responses by a specialized tropical bird. Biological Journal of the Linnean Society, 59:37-67.

Costa Lima, A.M. 1936. Terceiro catálogo dos insetos que vivem nas plantas cultivadas do Brasil. Ministério da Agricultura, Escola Nacional de Agronomia, Directoria Estatal de Produção, Rio de Janeiro, IV+460 pp.

Costa Lima, A.M. 1950. Insetos do Brasil. 6. Lepidópteros. 2a. parte, vol. 8. Escola Nacional de Agronomia, Rio de Janeiro, série didática.

D’Abrera, B. 1987. Butterflies of Neotropical Region. Part III. Brassolidae, Acraeidae and Nymphalidae (Partim). Hill House, Victoria, Australia.

D’Almeida, R.F. 1922. Mélanges lépidoptèrologiques. Etudes sur les lépidoptères du Brésil. R. Friedländler \& Sohn, Berlin, 1:VIII+226 pp.

D’Almeida, R.F. 1923. Notes sur quelques Lépidoptères rhopalocères du Brésil. III. Les Actinote de Rio. Annales de la Société entomologique de France, Paris, 91:229-235.

D’Almeida, R.F. 1925. Quelques rectifications sur les Actinote de la partie orientale de l'Amérique du Sud (Lép.Rhop.). Annales de la Société entomologique de France, Paris, 94:333-354.

D’Almeida, R.F. 1931. Beiträge zur Schemetterlings-Fauna Süd-Amerikas. Entomologische Zeitschrift, 45:59-61.

D’Almeida, R.F. 1934. Zwei neue Schmetterlinge aus Brasilien. Entomologische Zeitschrift 48(12): 90-92.

D’Almeida, R.F. 1935a. Les Actinote de la partie orientale de l'Amérique du Sud. Annaes da Academia Brasileira de Sciências, Rio de Janeiro, 7:69-112.
D’Almeida, R.F. 1935b. Nota suplementar ao nosso artigo sobre o gênero Actinote Hubn. Revista de Entomologia, Rio de Janeiro, 5:486-488.

D’Almeida, R.F. 1943. Algumas observações sobre o Actinote morio Oberthür, 1917 (Lep. Heliconidae, Acraeinae). Papéis Avulsos do Departamento de Zoologia, São Paulo, 3(16):107-109.

D’Almeida, R.F. 1951. Uma nova espécie de Actinote do Sul do Brasil (Lep. Heliconiidae, Acraeinae). Arquivos do Museu Nacional, Rio de Janeiro, (42):1-5.

D’Almeida, R.F. 1958. Ligeiras notas sobre algumas Actinote do sudeste do Brasil (Lep. Rhop.). Boletim do Museu Nacional, Rio de Janeiro (N. S.), Zoologia, 178:1-7.

D’Araujo e Silva, A.G., C.R. Gonçalves, D.M. Galvão, A.J.L. Gonçalves, J. Gomes, M.N. Silva \& L. Simoni. 1967-1968. Quarto catálogo dos insetos que vivem nas plantas do Brasil. Seus parasitas e predadores. Laboratório Central de Patologia Vegetal do Ministério da Agricultura, Rio de Janeiro. 1(1):XV+422 pp; 1(2):423-926 pp; 2(1):XXVII+622 pp; 2(2):VIII+265pp.

Ebert, H. 1969. On the frequency of butterflies in Eastern Brazil, with a list of the butterfly fauna of Poços de Caldas, Minas Gerais. Journal of the Lepidopterist's Society, 23(suppl. 3):1-48.

Ehrlich, A.H. \& P.R. Ehrlich. 1978. Reproductive strategies in the butterflies. I. Mating frequency, plugging and egg number. Journal of the Kansas Entomological Society, 51(4):666-697.

Ehrlich, P.R. 1958. The comparative morphology, phylogeny and higher classification of butterflies. Kansas University Scientific Bulletin, 39:305-370.

Eltringham, H. \& K. Jordan. 1913. Nymphalidae: subfam. Acraeinae. In: H.Wagner (ed.), Lepidopterorum catalogus, 11:1-65. W. Junk, Berlin.

Fabricius, J.C. 1775. Systema Entomologiae, sistens insectorvm classes, ordines, genera, species, adiectis synonymis, locis, descriptionibvs, observationibvs. Kortii, Flensburgi et Lipsiae, I + XXX, 832 pp.

Fabricius, J.C. 1781-1782. Species Insectorvm exhibentes eorvm differentias specificas, synonyma avctorvm, loca natalia, metamorphosin adiectis observationibvs descriptionibvs. Karl E. Bohn, Hamburgi et Kilonii, 1:1552 pp. (1781), 2:1-494 pp. (1781), appendix, pp. 494-514 (1782) and index pp. 515-517 (1782).

Fabricius, J.C. 1787. Mantissa insectorvm sistens species nvper detectas adiectis synonymis, observationibvs, descriptionibvs, emendationibvs. Christ. Gottl. Proft, Hafniae, 2:1-382.

Fabricius, J.C. 1793. Entomologia systematica emendata et aucta. Secundum classes, ordines, genera, species, adjectis synonimis, locis, observationibvs descriptionibvs. C.G.Proft, Hafniae, 3(1);VI, 488 pp. 
Felder, C. 1862. Verzeichniss der von den Naturforschern der K. K. Fregate "Novara” gesammelten Macrolepidopteren. Verhandlungen der kaiserlichköniglichen zoologisch-botanischem Gesellschaft in Wien, 12:473-496.

Felder, C. \& R. Felder. 1860. Lepidopterologische Fragmente. V. Wiener Entomologische Monatschrift, 4(4):97-112, 2 plates.

Fernandes, A.T., A.B.M. Machado \& A.B. Rylands. 1990. Fauna brasileira ameaçada de extinção. Fundação Biodiversitas / IBAMA, Belo Horizonte.

Francini, R.B. 1985. Ecologia de populações de Actinote do litoral de São Paulo (Lepidoptera: Nymphalidae). Resumos do XII Congresso Brasileiro de Zoologia, Campinas, SP, pp 104-105.

Francini, R.B. 1989. Biologia e ecologia das borboletas Actinote (Lepidoptera: Nymphalidae) na transição subtropical no sudeste do Brasil. M.S. Thesis, Universidade Estadual de Campinas, Campinas, Brazil.

Francini, R.B. 1992. Ecologia de Taxocenoses de Actinote (Lepidoptera: Nymphalidae) em Asteraceae (Angiosperma: Magnoliatae) no Sudeste do Brasil: Subsídios para Conservação. Ph.D. Dissertation, Universidade Estadual de Campinas, Campinas, Brazil.

Francini, R.B., A.V.L. Freitas \& C.M. Penz. 2004. Two new species of Actinote (Lepidoptera, Nymphalidae) from Southeastern Brazil. Zootaxa, 719:1-10.

Gabriel, A.G. 1927. Catalogue of the type speciemens of Lepidoptera Rhopalocera in the British Museum. III. Nymphalidae. British Museum, Oxford University Press, London, 128 pp.

Geyer, C.M. 1832. In: J. Hubner, Zuträge zur Sammlung exostischer Schmmetterlinge. J. Hübner, Augsburg, 5:1-48.

Gmelin, J.F. 1790. Caroli A. Linné Systema naturae per regna tria naturae, editio 13 . Beer, Lipsiae, volume 1(5)[2225-3020].

Godart, J.B. 1819-1824. In: P.A. Latreille \& J.B. Godart, Encyclopedie Methodique. Histoire naturelle. Aagasse, Paris. 9:1-828.

Goeze, J.A.E. 1779. Entomologische Beyträge zu des Ritter Linné zwölften Ausgabe des Natursystems. Weidmann, Leipzig, 3(1), XL + 390 pp.

Gosse, P.H. 1880. The butterflies of Paraguay and La Plata. Entomologist, London, 13(208):193-205, 1 plate.

Haase, E. 1893. Untersunchungen über die Mimicry auf Grundlage eines natürlichen Systema der Papilioniden. Bibliotheca Zoologica. Stuttgart, 8(11):99-161.

Hayward, K.J. 1931. Lepidópteros argentinos. Familia Nymphalidae. Revista de la Sociedad Entomológica Argentina, Buenos Aires, 4(1-3):1-199.
Hayward, K.J. 1935. Revisión de las especies argentinas del género Actinote (Lep. Nymphalidae). Revista de la Sociedad Entomológica Argentina, Buenos Aires, 7:93-97.

Hayward, K.J. 1951. Catálogo sinonímico de los ropalóceros argentinos excluyendo “Hesperidae”. Acta Zoologica Lilloana, Tucumán, 9:85-281.

Hayward, K.J. 1968. Lista de los tipos de insectos y otros invertebrados conservados en el Instituto Miguel Lillo (tercera entrega). Acta Zoologica Lilloana, Tucumán, 22:337-352.

Hemming, F. 1967. The generic names of the butterflies and their type-species (Lepidoptera: Rhopalocera). Bulletin of the British Museum (Natural History) Entomology Supplement 9:1-509.

Hopffer, C. 1874. Neue Lepidopteren von Peru und Bolivia. Stettiner Entomologische Zeitung, 35(10-12):329-371.

Hübner, J. 1816-1826. Verzeischniss bekannter Schmettlinge. J. Hübner, Augsburg, $431+72$ pp.

Jones, E. D. \& F. Moore. 1883. Metamorphoses of Lepidoptera from S.Paulo, Brazil, in the Free Museum, Liverpool, by E. Dukinfield Jones, with nomenclature and descriptions of new forms by F. Moore, and introductory note by T.J. Moore. Proceedings of the Literary and Philosophical Society of Liverpool, 2:2173, plates 3-6.

Jordan, K. 1913a. Diagnoses of some American Acraeinae. Entomologist, London, 46:32-33.

Jordan, K. 1913b. Acraeinae. In: A. Seitz (ed.), GrossSchmetterlinge der Erde. Die amerikanischen Tagfalter, vol. 5, pp. 358-374, plates 81-83. Alfred Kernen, Stuttgart.

Jordan, K. \& H.H. Eltringham. 1916. Lepidoptera Rhopalocera, Fam. Nymphalidae, subfam. Acraeinae. In: P. Wytsman (ed.), Genera Insectorum, 169:1-81, 2 plates. Martinus Nijhoff, La Haye.

Joseph, E.G. 1911. The collections of William John Burchell, D.C.L., in the Hope Department of the Oxford University Museum. IV. On the Lepidoptera Rhopalocera collected by W.J. Burchell in Brazil, 18251830. VIII. Acraeinae. Annals and Magazine of Natural History, London, 8(7):9-18.

Kirby, W.F. 1871. A synonymic catalogue of diurnal Lepidoptera. Van Voorst, London, VII + 690 pp.

Lamas, G. 1973. The type-material of Lepidoptera Rhopalocera contained in the collections of the Museu de Zoologia da Universidade de São Paulo. Papéis Avulsos de Zoologia, São Paulo, 26(13):179-185.

Lamas, G. 1996. Lista comentada de los nombres propuestos para los Acraeini neotropicales, y su material-tipo (Lepidoptera: Nymphalidae, Heliconiinae). Rrevista Peruana de Entomologia, Lima, 39:29-48. 
Lamas, G. 2004. Nymphalidae. Heliconiinae, pp. 261-274. In: Lamas, G. (Ed.), Checklist: Part 4A. Hesperioidea Papilionoidea. In: Heppner, J. B. (Ed.), Atlas of Neotropical Lepidoptera. Volume 5A. Association for Tropical Lepidoptera/Scientific Publishers, Gainesville.

Lewis, H.L. 1975. Las mariposas del mundo. Ediciones Omega, Barcelona.

Lucas, P.H.. 1835. Histoire naturelle des lépidoptères exotiques. Pauquet, Paris, IV + 156 pp., 80 plates.

Mabilde, A.P. 1896. Borboletas do Estado do Rio Grande do Sul. Guia prática para os principiantes colleccionadores de insectos contendo a descripção fiel de perto de 1000 borboletas com 280 figuras lythographadas em tamanho, formas e dezenhos conforme o natural. Estudo sobre a vida de insectos do Rio Grande do Sul e sobre a caça, classificação e conservação de uma collecção, mais ou menos regular. Typographia Gundlach \& Schuldt, Porto Alegre, 240 pp., 24 plates.

Mielke, O.H.H. \& M.M. Casagrande. 1986. Sobre os tipos de Lepidoptera depositados em museus brasileiros. III. Nymphalidae (Danainae, Brassolinae, Morphinae, Satyrinae Nymphalinae e Acraeinae), descritos por R.F. D’Almeida. Revista Brasileira de Entomologia, 30(1):141-152.

Monte, O. 1934. Borboletas que vivem em plantas cultivadas. Secretaria de Agricultura de Minas Gerais, Série Agrícola, 21:VIII + 221 pp.

Müller, F. 1876. Einige Worte über Leptalis. Jenaische Zeitschrift für Naturwissenschaft, 10:1-12.

Müller, F. 1877a. Notes on Brazilian entomology. Transactions of the Entomological Society of London, 1877(3):211-223.

Müller, F. 1877b. Beobachtungen an brasilianischen Schmetterlingen. III. Kosmos, Leipzig, 2:218-224.

Müller, F. 1878a. Pflanzengattungen, an denem mir bekannte Tagfalter-Raupen leben. Stettiner Entomologische Zeitung, 39:296.

Müller, F. 1878b. Ueber die Vortheile der Mimicry bei Schmetterlingen. Zoologischer Anzeiger, 1:54-55.

Müller, F. 1879. On a remarkable case of mimicry of Eueides pavana with Acraea thalia. Transactions of the Entomological Society of London, 1879(2):XX-XXIV.

Müller, F. 1882. Bemerkenswerte Fälle erworbener Aehnlichkeit bei Schmetterlingen. Kosmos, Stuttgart, 10:257-267.

Müller, F. 1883a. Der Anhang am Hinterleib der AcraeaWeibchen. Zoologische Anzeiger, 6:415-416.

Müller, F. 1883b. Eine Aufgabe für Lepidopterologen. Berliner Entomologische Zeitschrift, 27(2):214-216.

Müller, F. 1883c. Angebissene Flügel von Acraea thalia. Kosmos, Stuttgart, 13:197-201.
Müller, F. 1915. In: A. Möller (ed.) Fritz Müller: Werke, Briefe und Leben. Jena, 2 vols.

Müller, W. 1886. Südamerikanische Nymphalidenraupen. Versuch eines natürlichen Systems der Nymphaliden. Zoologische Jahrbücher. Abteilung für Systematik Geographie und Biologie der Tiere, 1:417-678, plates 12-15.

Oberthür, C. 1917. Le genre Actinote. Études de Lépidoptèrologie Comparée, 14:77-125, 12 plates.

Orfila, R.N. 1964. Una nueva plaga para la Argentina: "la isoca espinosa del girassol”, Actinote pellenea pellenea Hübner (Lep. Acraeidae). Idia, Buenos Aires, 196:41-48.

Paluch, M.; M.M. Casagrande \& O.H.H. Mielke. 1999. Estágios imaturos de Actinote surima (Schaus) (Lepidoptera, Nymphalidae, Acraeinae) Revista Brasileira de Zoologia, Curitiba, 16(supl. 2): 129-140.

Paluch, M., M.M. Casagrande \& O.H.H. Mielke. 2001. Estágios imaturos de Actinote carycina Jordan (Lepidoptera: Nymphalidae: Acraeinae). Revista Brasileira de Zoologia, Curitiba, 18(3):883-896.

Paluch, M., M.M. Casagrande \& O.H.H. Mielke. 2003. Tampão genital de Actinote Hübner, como caráter taxonômico (Lepidoptera, Nymphalidae, Acraeinae). Revista Brasileira de entomologia, 47:573-580.

Penz, C.M. 1983. O gênero Actinote Hübner, 1819 no sul do Brasil (Lep., Nymph., Acraeinae). B.S. Thesis, Universidade Federal do Rio Grande do Sul, Porto Alegre, Brazil.

Penz, C.M. 1985. Estudos sobre as espécies de Actinote Hübner, 1819 do Rio Grande do Sul (Lep., Nymph., Acraeinae). Resumos do XII Congresso Brasileiro de Zoologia, Campinas, p. 104.

Penz, C.M. \& P. Djunijanti. 2003. Phylogenetic relationships among Heliconiinae genera based on early stage and adult morphology (Lepidoptera, Nymphalidae). Systematic Entomology, 28:451-479.

Penz, C.M. \& R.B. Francini. 1996. New species of Actinote Hübner (Nymphalidae: Acraeinae) from Southeastern Brazil. Journal of the Lepidopterists' Society, 50:309320.

Pierre, J. 1983. Systématique évolutive, cladistique et mimétisme chez les lépidoptères du genre Acraea (Nymphalidae). PhD Dissertation, University of Paris, Paris, France.

Pierre, J. 1987 Systématique cladistique chez les Acraea (Lepidoptera, Nymphalidae). Annales de la Société Entomologique de France (N.S.), 23(1):11-27.

Prittwitz, O. 1865. Beiträge zur Fauna des Corcovado. Stettiner Entomologische Zeitung, 26:123-143.

Ronna, E. 1934. Primeiro ensaio da catalogação dos insetos do Brasil auxiliares na luta contra as pragas. O Campo, Rio de Janeiro, 5(7):33-36. 
Schaus, W. 1902. Descriptions of new American butterflies. Proceedings of the United States National Museum, 24(1262):383-460.

Smart, P. 1976. Encyclopédie des papillons. Elsevier Sequoia, Bruxelles.

Srygley, R. B. 1994. Locomotor mimicry in butterflies? The associations of positions of centres of mass among groups of mimetic, unprofitable prey. Philosophical Transactions of the Royal Society, London, B, 343:145-155.

Staudinger, O. 1884-1888. Exotische tagfalter in systematischer Reihenfolge mit Berücksichtigung neuer Arten. In: Staudinger, O. \& E. Schatz, Exotische Schmetterlinge, vol 1(1): 333 pp + 1 map; vol 1(2): 100 plates. G. Lowensohn, Fürth, Bayern.

Toledo, Z.D.A. 1980. Fauna del NOA. Contribución al conocimiento de los lepidópteros argentinos. VIII. Actinote diaguita Hayward (Lepidoptera, Acracidae [sic]). Acta Zoologica Lilloana, 36(1):87-93.

Turner, J. R. G. 1987. The evolutionary dynamics of Batesian and Mullerian mimicry: similarities and differences. Ecological Entomology, 12:81-95.

Van Son, G. 1963. The Butterflies of the Southern Africa. Part 3: Nymphalidae: Acraeinae. Transvaal Museum, Pretoria.

Weymer, G. 1894 Exotische Lepidopteren. VII. Beitrag zur Lepidopterenfauna von Rio Grande do Sul. Stettiner Entomologische Zeitung, 55(10-12):311-333.
Title: An illustrated key to male Actinote from Southeastern Brazil (Lepidoptera, Nymphalidae).

Authors: Ronaldo Bastos Francini \& Carla M. Penz

Biota Neotropica, Vol. 6 ( number 1): 2006

http://www.biotaneotropica.org.br/v6n1/pt/ abstract?identification-key+bn00606012006

Date Received 06/06/2005 - Revised10/20/2005

Accepted 01/01/2006

ISSN 1676-0611 